\title{
Identification of Femoral-Acetabular Symptoms Using sEMG Signals During Dynamic Contraction
} by

\author{
Zahra Karimi Tabar
}

A thesis submitted to The Faculty of Graduate and Postdoctoral Affairs in partial fulfillment of the requirements for the degree of Master of Applied Science in Biomedical Engineering
Ottawa-Carleton Institute for Biomedical Engineering (OCIBE)
Department of Systems and Computer Engineering Carleton University

Ottawa, Ontario, Canada, K1S 5B6

Copyright 2014, Zahra Karimi Tabar 
The undersigned recommend to

the Faculty of Graduate and Postdoctoral Affairs

acceptance of the thesis

\title{
Identification of Femoral-Acetabular Symptoms Using sEMG Signals During Dynamic Contraction
}

\author{
submitted by \\ Zahra Karimi Tabar, B.A.Sc. \\ in partial fulfillment of the requirements for \\ the degree of Master of Applied Science in Biomedical Engineering
}

Chair, Roshdy H. M. Hafez, Department of Systems and Computer Engineering

Thesis Supervisor, Chris Joslin

Carleton University

May 2014 


\section{Abstract}

This thesis focuses on development of an algorithm that automatically identifies a Femoroacetabular Impingement (FAI) patient from a healthy control person by comparing their surface electromyography (sEMG) signal recorded from Gluteus Maximus (GMax), Tensor Fasciae Latae (TFL), and Rectus Femoris (RF) muscles in the hip area. A discrete wavelet transform (DWT) method was used to analyze sEMG signals by thirty eight different wavelet functions (WFs) with 5 decomposition levels of dynamic contractions during the three phases (descending, stationary, and ascending) of squat task. The Bior3.9 WF was selected as it provided higher amount of energy for most of the subjects and then the wavelet power spectrum was computed for healthy control and FAI groups. The results show that the RF muscle is more active in the ascending phase than the descending phase for FAI subjects, whereas it is more active in the descending phase for healthy control. An independent sample t-test was used to check the activities of muscle in both groups. The results demonstrate no significant difference for GMax $(\mathrm{p}=0.7477)$ and TFL $(\mathrm{P}=0.4997)$ muscles, while there is a significant difference for RF muscle $(\mathrm{p}=0.0670)$. 


\section{Acknowledgments}

I would like to express my deep gratitude and respect to Dr. Chris Joslin, my supervisor, for his continuous help and support in all stages of this thesis work. I would also like to thank him for his tireless efforts and being an open person to ideas in my research and encouraging and helping me in all steps. Furthermore, I really would like to thank Dr. Mario Lamontagne for his time and constructive comments.

I want to thank Giulia Mantovani whose advice and insight was invaluable to me. For all I learned from her and for providing the data experiment for my research. I also appreciate Dr. Richard Dansereau for his assistance on the topic of wavelet transforms.

I would like to thank my parents, Hadi Karimi Tabar and Maryam Tavasouli Amin, and my sister Narges Karimi Tabar. I am very thankful to my brother-in-law Mohammad Reza Pasandideh for his encouragement and support.

I am indebted to my friend, Mehdi Mostajeran, for all hope and energy he gave me to complete this thesis.

I am grateful to Peyman Rahmati for very fruitful discussions that assisted me to solve some of my research problems. 


\section{Contents}

1 Introduction 1

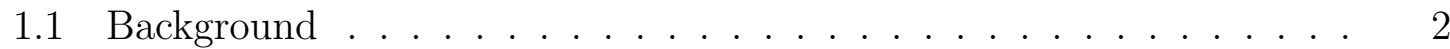

1.1.1 Anatomy of the Hip Joint . . . . . . . . . . . . . . 2

1.1.2 Femoroacetabular impingement ............ 5

1.1.3 Skeletal Muscle Physiology . . . . . . . . . . . . . . . 7

1.1.4 EMG Signals . . . . . . . . . . . . . . . . 9

1.2 Problem Statement . . . . . . . . . . . . . . . . . . 11

1.3 Research Objectives . . . . . . . . . . . . . . . . . . . . . 12

1.4 Methodology . . . . . . . . . . . . . . . . . 13

1.5 Contribution ........................... 14

1.6 Thesis Overview . . . . . . . . . . . . . . . . . . 15

2 Literature Review $\quad 16$

2.1 Background ........................... 16

2.2 Muscle weakness in FAI . . . . . . . . . . . . . . . 17

2.3 Time-Frequency Analysis Methods . . . . . . . . . . . . . 20

2.3.1 Wavelet Transforms ................ 23 
2.3.1.1 Continuous Wavelet Transform . . . . . . . . . 25

2.3.1.2 Discrete Wavelet Transform . . . . . . . . . . . 26

2.3.2 Wavelet Types and Families . . . . . . . . . . . . . . . . 28

2.3.2.1 Properties of Wavelet Functions . . . . . . . . . . . . 28

2.3.3 Choosing a Wavelet for EMG Signals . . . . . . . . . . . . 36

2.3.4 Different Studies using the Wavelet Transform . . . . . . . . . 38

3 Methodology 43

3.1 Recording Process for EMG Signals . . . . . . . . . . . . . . . . . . 44

3.2 EMG Data Processing . . . . . . . . . . . . . . . . 47

3.3 EMG Analysis Using Wavelet Transforms . . . . . . . . . . . . . . . . 51

3.3.1 Level of Decomposition . . . . . . . . . . . . . . . . 52

3.3.2 Multi-Resolution Analysis .............. 53

3.3.3 Wavelet Selection Based on Energy Calculation . . . . . . . . 53

3.3.4 Wavelet Power Spectrum . . . . . . . . . . . . 54

4 Analysis of Results $\quad 55$

4.1 Data Selection . . . . . . . . . . . . . . . 56

4.2 Wavelet Transforms . . . . . . . . . . . . . . . . . . 61

4.2.1 Selecting a Wavelet Function . . . . . . . . . . . . . . . . 62

4.2.2 Principal Component Analysis . . . . . . . . . . . . . . . . . . 72

4.2.3 Bior3.9 Energy and Weight .............. 73

4.2.4 Average Energy . . . . . . . . . . . . . . . . 82

4.2.5 Wavelet Power Spectrum . . . . . . . . . . . . . 84

4.2.6 Algorithm ...................... 101 
4.3 Statistical Analysis . . . . . . . . . . . . . . . . . 102

4.4 Discussion . . . . . . . . . . . . . . . . . . . . 102

5 Conclusions and Future Work 105

$\begin{array}{lr}\text { Appendix A } & 107\end{array}$

$\begin{array}{lr}\text { References } & 141\end{array}$ 


\section{List of Tables}

3.1 Participant Characteristics by Group . . . . . . . . . . . . . 46

3.2 Participants' MVIC Values for GMax, TFL, and RF Muscles . . . . 48

4.1 Energy of Muscle3 During Descending Phase for One CON Subject . 63

4.2 Energy of Muscle3 During Descending Phase for One OR Subject . . 64

4.3 Five Wavelet Functions With Highest Energy for Muscle 3 During Descending Phase of One CON Subject . . . . . . . . . 65

4.4 Five Wavelet Functions With Highest Energy for Muscle 3 During Descending Phase of One OR Subject . . . . . . . . . 65

4.5 Selection of a WF With Highest Repetition of Energy for Muscle 1-CON 66

4.6 Selection of a WF With Highest Repetition of Energy for Muscle 1-OR 67

4.7 Selection of a WF With Highest Repetition of Energy for Muscle 2-CON 68

4.8 Selection of a WF With Highest Repetition of Energy for Muscle 2-OR 69

4.9 Selection of a WF With Highest Repetition of Energy for Muscle 3-CON 70

4.10 Selection of a WF With Highest Repetition of Energy for Muscle 3-OR 71

4.11 Example of Wavelet Power Spectrum Calculations for Muscle 1 of One

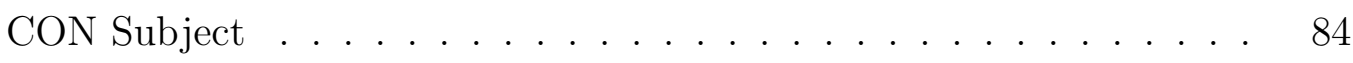


4.12 Example of Wavelet Power Spectrum Calculations for Muscle 2 of One

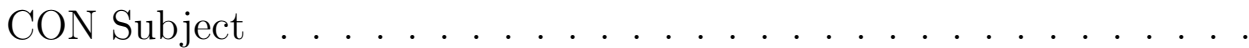

4.13 Example of Wavelet Power Spectrum Calculations for Muscle 3 of One CON Subject . . . . . . . . . . . . . . . .

4.14 Example of Wavelet Power Spectrum Calculations for Muscle 1 of One

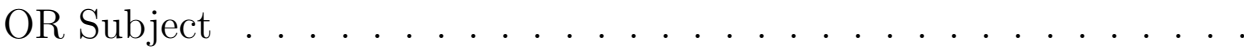

4.15 Example of Wavelet Power Spectrum Calculations for Muscle 2 of one OR Subject ....................... 86

4.16 Example of Wavelet Power Spectrum Calculations for Muscle 3 of One

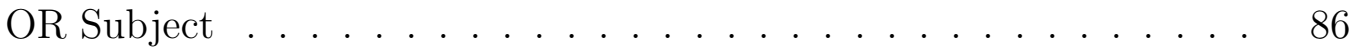

4.17 Muscles used by CON and OR During Squat Cycle . . . . . . . . . . 93

4.18 Ratio A/D Phase for One CON Subject . . . . . . . . . . . . . . 99

4.19 Ratio A/D Phase for One OR Subject . . . . . . . . . . . . . 99

4.20 Average Ratio With Standard Deviation Values for Each Muscle in $\mathrm{CON}$ and $\mathrm{OR}$ Groups . . . . . . . . . . . . . . . 100

4.21 P-value for GMax, TFL, and RF Muscle . . . . . . . . . . . . . . 102

A.1 IEMG and Time Normalized Values for Muscle 1 Without Outliers . 108

A.2 IEMG and Time Normalized Values for Muscle 2 Without Outliers . 109

A.3 IEMG and Time Normalized Values for Muscle 3 Without Outliers . 110

A.4 Average Energy for Muscle 1 of CON and OR Group . . . . . . . . . 111

A.5 Average Energy for Muscle 2 of CON and OR Group . . . . . . . . . 112

A.6 Average Energy for Muscle 3 of CON and OR Group . . . . . . . . . 113

A.7 Wavelet Power Spectrum Calculations for Muscle 1-CON Subjects 1 . 114 
A.8 Wavelet Power Spectrum Calculations for Muscle 1-CON Subjects 2 . 114

A.9 Wavelet Power Spectrum Calculations for Muscle 1-CON Subjects 3 . 114

A.10 Wavelet Power Spectrum Calculations for Muscle 1-CON Subjects 4 . 114

A.11 Wavelet Power Spectrum Calculations for Muscle 1-CON Subjects 5 . 115

A.12 Wavelet Power Spectrum Calculations for Muscle 1-CON Subjects 6 . 115

A.13 Wavelet Power Spectrum Calculations for Muscle 1-CON Subjects 7 . 115

A.14 Wavelet Power Spectrum Calculations for Muscle 1-CON Subjects 8 . 115

A.15 Wavelet Power Spectrum Calculations for Muscle 1-CON Subjects 9 . 115

A.16 Wavelet Power Spectrum Calculations for Muscle 1-CON Subjects 10116

A.17 Wavelet Power Spectrum Calculations for Muscle 1-CON Subjects $11 \quad 116$

A.18 Wavelet Power Spectrum Calculations for Muscle 1-CON Subjects 12116

A.19 Wavelet Power Spectrum Calculations for Muscle 1-CON Subjects 13116

A.20 Wavelet Power Spectrum Calculations for Muscle 1-CON Subjects 14116

A.21 Wavelet Power Spectrum Calculations for Muscle 1-CON Subjects $15 \quad 117$

A.22 Wavelet Power Spectrum Calculations for Muscle 1-CON Subjects $16 \quad 117$

A.23 Wavelet Power Spectrum Calculations for Muscle 1-OR Subjects 1 . . 118

A.24 Wavelet Power Spectrum Calculations for Muscle 1-OR Subjects 2 . . 118

A.25 Wavelet Power Spectrum Calculations for Muscle 1-OR Subjects 3 . . 118

A.26 Wavelet Power Spectrum Calculations for Muscle 1-OR Subjects 4 . . 118

A.27 Wavelet Power Spectrum Calculations for Muscle 1-OR Subjects 5 . . 119

A.28 Wavelet Power Spectrum Calculations for Muscle 1-OR Subjects 6 . . 119

A.29 Wavelet Power Spectrum Calculations for Muscle 1-OR Subjects 7 . . 119

A.30 Wavelet Power Spectrum Calculations for Muscle 1-OR Subjects 8 . . 119

A.31 Wavelet Power Spectrum Calculations for Muscle 1-OR Subjects 9 . . 120 
A.32 Wavelet Power Spectrum Calculations for Muscle 1-OR Subjects 10 . 120

A.33 Wavelet Power Spectrum Calculations for Muscle 1-OR Subjects 11 . 120

A.34 Wavelet Power Spectrum Calculations for Muscle 1-OR Subjects 12 • 120

A.35 Wavelet Power Spectrum Calculations for Muscle 2-CON Subjects 1 . 121

A.36 Wavelet Power Spectrum Calculations for Muscle 2-CON Subjects 2 . 121

A.37 Wavelet Power Spectrum Calculations for Muscle 2-CON Subjects 3 . 121

A.38 Wavelet Power Spectrum Calculations for Muscle 2-CON Subjects 4 . 121

A.39 Wavelet Power Spectrum Calculations for Muscle 2-CON Subjects 5 . 122

A.40 Wavelet Power Spectrum Calculations for Muscle 2-CON Subjects 6 . 122

A.41 Wavelet Power Spectrum Calculations for Muscle 2-CON Subjects 7 . 122

A.42 Wavelet Power Spectrum Calculations for Muscle 2-CON Subjects 8 . 122

A.43 Wavelet Power Spectrum Calculations for Muscle 2-CON Subjects 9 • 122

A.44 Wavelet Power Spectrum Calculations for Muscle 2-CON Subjects 10123

A.45 Wavelet Power Spectrum Calculations for Muscle 2-CON Subjects $11 \quad 123$

A.46 Wavelet Power Spectrum Calculations for Muscle 2-CON Subjects 12123

A.47 Wavelet Power Spectrum Calculations for Muscle 2-CON Subjects 13123

A.48 Wavelet Power Spectrum Calculations for Muscle 2-CON Subjects 14124

A.49 Wavelet Power Spectrum Calculations for Muscle 2-CON Subjects 15124

A.50 Wavelet Power Spectrum Calculations for Muscle 2-CON Subjects $16 \quad 124$

A.51 Wavelet Power Spectrum Calculations for Muscle 2-OR Subjects 1 . . 125

A.52 Wavelet Power Spectrum Calculations for Muscle 2-OR Subjects 2 . . 125

A.53 Wavelet Power Spectrum Calculations for Muscle 2-OR Subjects 3 . . 125

A.54 Wavelet Power Spectrum Calculations for Muscle 2-OR Subjects 4 . . 125

A.55 Wavelet Power Spectrum Calculations for Muscle 2-OR Subjects 5 . . 126 
A.56 Wavelet Power Spectrum Calculations for Muscle 2-OR Subjects 6 . . 126

A.57 Wavelet Power Spectrum Calculations for Muscle 2-OR Subjects 7 . . 126

A.58 Wavelet Power Spectrum Calculations for Muscle 2-OR Subjects 8 . . 126

A.59 Wavelet Power Spectrum Calculations for Muscle 2-OR Subjects 9 . . 126

A.60 Wavelet Power Spectrum Calculations for Muscle 2-OR Subjects 10 . 127

A.61 Wavelet Power Spectrum Calculations for Muscle 2-OR Subjects 11 • 127

A.62 Wavelet Power Spectrum Calculations for Muscle 2-OR Subjects 12 . 127

A.63 Wavelet Power Spectrum Calculations for Muscle 3-CON Subjects 1 . 128

A.64 Wavelet Power Spectrum Calculations for Muscle 3-CON Subjects 2 . 128

A.65 Wavelet Power Spectrum Calculations for Muscle 3-CON Subjects 3 . 128

A.66 Wavelet Power Spectrum Calculations for Muscle 3-CON Subjects 4 . 128

A.67 Wavelet Power Spectrum Calculations for Muscle 3-CON Subjects 5 • 129

A.68 Wavelet Power Spectrum Calculations for Muscle 3-CON Subjects 6 . 129

A.69 Wavelet Power Spectrum Calculations for Muscle 3-CON Subjects 7 . 129

A.70 Wavelet Power Spectrum Calculations for Muscle 3-CON Subjects 8 . 129

A.71 Wavelet Power Spectrum Calculations for Muscle 3-CON Subjects 9 . 129

A.72 Wavelet Power Spectrum Calculations for Muscle 3-CON Subjects $10 \quad 130$

A.73 Wavelet Power Spectrum Calculations for Muscle 3-CON Subjects $11 \quad 130$

A.74 Wavelet Power Spectrum Calculations for Muscle 3-CON Subjects $12 \quad 130$

A.75 Wavelet Power Spectrum Calculations for Muscle 3-CON Subjects $13 \quad 130$

A.76 Wavelet Power Spectrum Calculations for Muscle 3-CON Subjects $14 \quad 130$

A.77 Wavelet Power Spectrum Calculations for Muscle 3-CON Subjects 15131

A.78 Wavelet Power Spectrum Calculations for Muscle 3-CON Subjects 16131

A.79 Wavelet Power Spectrum Calculations for Muscle 3-OR Subjects 1 . . 132 
A.80 Wavelet Power Spectrum Calculations for Muscle 3-OR Subjects 2 . . 132

A.81 Wavelet Power Spectrum Calculations for Muscle 3-OR Subjects 3 . . 132

A.82 Wavelet Power Spectrum Calculations for Muscle 3-OR Subjects 4 . . 132

A.83 Wavelet Power Spectrum Calculations for Muscle 3-OR Subjects 5 . . 133

A.84 Wavelet Power Spectrum Calculations for Muscle 3-OR Subjects 6 . . 133

A.85 Wavelet Power Spectrum Calculations for Muscle 3-OR Subjects 7 . . 133

A.86 Wavelet Power Spectrum Calculations for Muscle 3-OR Subjects 8 . . 133

A.87 Wavelet Power Spectrum Calculations for Muscle 3-OR Subjects 9 . . 133

A.88 Wavelet Power Spectrum Calculations for Muscle 3-OR Subjects 10 . 134

A.89 Wavelet Power Spectrum Calculations for Muscle 3-OR Subjects 11 • 134

A.90 Wavelet Power Spectrum Calculations for Muscle 3-OR Subjects 12 • 134

A.91 Ratio of A/D for Muscle 1 of CON subjects . . . . . . . . . . 135

A.92 Ratio of A/D for Muscle 1 of OR subjects . . . . . . . . . . . 136

A.93 Ratio of A/D for Muscle 2 of CON subjects . . . . . . . . . . 137

A.94 Ratio of A/D for Muscle 2 of OR subjects . . . . . . . . . 138

A.95 Ratio of A/D for Muscle 3 of CON subjects . . . . . . . . . . . 139

A.96 Ratio of A/D for Muscle 3 of OR subjects . . . . . . . . . . . 140 


\section{List of Figures}

1.1 A Healthy Hip, Femoral Head Fits into the Acetabulum [1] . . . . . . 3

1.2 Muscles in Hip Area [2] . . . . . . . . . . . . . . . . . . 4

1.3 FAI Forms $[3] \ldots \ldots \ldots \ldots$

1.4 Overview of Skeletal Muscle Structure [4] . . . . . . . . . . . . . . 7

1.5 Sliding-Filament Model of Muscle Contraction . . . . . . . . . . . . . 9

1.6 Motor Unit . . . . . . . . . . . . . . . . . . . . . 10

2.1 Time-Frequency Plane in STFT and WT [5] . . . . . . . . . . . 22

2.2 Wavelet Multi-Resolution Analysis [5] . . . . . . . . . . . . . . . . 27

2.3 Haar Scaling and Wavelet Function . . . . . . . . . . . . . . . . . . . 29

2.4 Daubechies 2 and 10 Scaling and Wavelet Functions . . . . . . . . . . 30

2.5 Symlets 8 Scaling and Wavelet Functions . . . . . . . . . . . . . . 31

2.6 Coiflets 2 and 5 Scaling and Wavelet Functions . . . . . . . . . . 32

2.7 Biorthogonal Decomposition Scaling and Wavelet Functions . . . . . 33

2.8 Morlet Wavelet . . . . . . . . . . . . . . . . . . . . . . 34

2.9 Mexican Hat Wavelet . . . . . . . . . . . . . . . . . . . . . . . . . . . 34

2.10 Meyer Scaling and Wavelet Function . . . . . . . . . . . 35 
3.1 Schematic Description of the Technique . . . . . . . . . . . . . . 44

3.2 Descending, Stationary, and Ascending Phase of Squat [6] . . . . . 45

3.3 Normalized EMG Signals for CON (blue) and OR (red), Muscle 1 . . 50

3.4 Normalized EMG Signals for CON (blue) and OR (red), Muscle 2 . . 50

3.5 Normalized EMG Signals for CON (blue) and OR (red), Muscle 3 . . 51

3.6 Procedure of Selecting a WF . . . . . . . . . . . . . . . 53

3.7 Block Diagram for Computing Power Spectrum . . . . . . . . . . . . 54

4.1 Integrated EMG Graphs for CON (blue) and OR (red), Muscle 1 . . 57

4.2 Integrated EMG Graphs for CON (blue) and OR (red), Muscle 2 . . 58

4.3 Integrated EMG Graphs for CON (blue) and OR (red), Muscle 3 . . 58

4.4 Time Normalized IEMG for Muscle 1 . . . . . . . . . . . . . . . . . . 59

4.5 Time Normalized IEMG for Muscle $2 \ldots \ldots$. . . . . . . . . . . 60

4.6 Time Normalized IEMG for Muscle $3 \ldots \ldots$. . . . . . . . . . . . . . 61

4.7 Bior3.9 Energy versus Weight for Muscle 1 in Descending Phase . . . 74

4.8 Bior3.9 Energy versus Weight for Muscle 1 in Stationary Phase . . . . 75

4.9 Bior3.9 Energy versus Weight for Muscle 1 in Ascending Phase . . . . 76

4.10 Bior3.9 Energy versus Weight for Muscle 2 in Descending Phase . . . 77

4.11 Bior3.9 Energy versus Weight for Muscle 2 in Stationary Phase . . . . 78

4.12 Bior3.9 Energy versus Weight for Muscle 2 in Ascending Phase . . . . 79

4.13 Bior3.9 Energy versus Weight for Muscle 3 in Descending Phase . . . 80

4.14 Bior3.9 Energy versus Weight for Muscle 3 in Stationary Phase . . . . 81

4.15 Bior3.9 Energy versus Weight for Muscle 3 in Ascending Phase . . . . 82

4.16 Average Energy for Muscle 1, 2, and $3 \ldots \ldots$. . . . . . . . . 83 
4.17 Example of Wavelet Power for Muscle 1 of One CON Subject . . . . 87

4.18 Example of Wavelet Power for Muscle 2 of One CON Subject . . . . 88

4.19 Example of Wavelet Power for Muscle 3 of One CON Subject . . . . 89

4.20 Example of Wavelet Power for Muscle 1 of One OR Subject . . . . . 90

4.21 Example of Wavelet Power for Muscle 2 of One OR Subject . . . . . 91

4.22 Example of Wavelet Power for Muscle 3 of One OR Subject . . . . . 92

4.23 Power of Muscle 1 for CON and OR during squat cycles . . . . . . . 94

4.24 Power of Muscle 2 for CON and OR during squat cycles . . . . . . . 95

4.25 Power of Muscle 3 for CON and OR during squat cycles . . . . . . 96

4.26 Muscles Power for CON Group . . . . . . . . . . . . . . . . 97

4.27 Muscles Power for OR Group . . . . . . . . . . . . . . . . . . 98

4.28 Average Ratio Muscle Power for CON and OR . . . . . . . . . . . . 101 


\section{Acronyms}

$\begin{array}{ll}\text { ANN } & \text { Artificial Neural Network } \\
\text { AP } & \text { Action Potential } \\
\text { AR } & \text { Auto-Regressive } \\
\text { BF } & \text { Biceps Femoris } \\
\text { BMI } & \text { Body Mass Index } \\
\text { CF } & \text { Center Frequency } \\
\text { CI } & \text { Co-activation Index } \\
\text { CWD } & \text { Chio-Williams Distribution } \\
\text { EMG } & \text { Electromyography } \\
\text { FAI } & \text { Femoroacetabular impingement } \\
\text { FFT } & \text { Fast Fourier Transform } \\
\text { FMF } & \text { Fourier Mean Frequency } \\
\text { FT } & \text { Fourier Transform } \\
\text { GMax } & \text { Gluteus Maximus } \\
\text { GRNN } & \text { General Regression Neural Networks } \\
\text { HHD } & \text { Hand-Held Dynamometer } \\
\text { IEMG } & \text { Integrated EMG } \\
& \end{array}$




\begin{tabular}{|c|c|}
\hline MDF & Median Frequency \\
\hline $\mathrm{ME}$ & Myoelectric \\
\hline MLP & Multilayer Perceptron \\
\hline MM & Mechanomyographic \\
\hline MMP & Multi-Muscle Pattern \\
\hline MPF & Mean Power Frequency \\
\hline MRI & Magnetic Resonance Imaging \\
\hline MU & Motor Unit \\
\hline MUAP & Motor Unit Action Potential \\
\hline MVIC & Maximum Voluntary Isometric Contraction \\
\hline $\mathrm{NN}$ & Neural Network \\
\hline PCA & Principal Component Analysis \\
\hline PSD & Power Spectral Density \\
\hline $\mathrm{RBF}$ & Radial Base Function \\
\hline $\mathrm{RF}$ & Rectus Femoris \\
\hline RMS & Root Mean Square \\
\hline sEMG & Surface Electromyography \\
\hline ST & Semitendinosus \\
\hline STFT & Short-Time Fourier Transform \\
\hline TFL & Fasciae Latae \\
\hline WF & Wavelet Function \\
\hline WMF & Wavelet Mean Frequency \\
\hline WT & Wavelet Transform \\
\hline WVD & Wigner-Ville Distribution \\
\hline
\end{tabular}




\section{Chapter 1}

\section{Introduction}

Femoroacetabular Impingement (FAI) is a pathomechanical process where the human hip can fail. FAI usually appears in young and active adults with pain in groin area, although many FAI patients have no history of hip disease $[7,8]$. Abnormalities of hip bones can damage the soft tissue structures and limit the patients' motion. Useful information can be achieved from the muscles and such information has clinical and engineering applications by measuring Electromyography (EMG) signals. EMG is a biomedical signal that provides a great source of information to clinicians and researchers by measuring the electrical currents generated in muscles during its contraction [9]. The EMG signal which is a complex signal is controlled by nervous system and the physiological and anatomical properties of muscles can influence on it $[10]$.

In this thesis, we are interested in the developing of an algorithm to discriminate a FAI patient from a healthy person by comparing their EMG signal recorded from hip muscles. As a result, this introduction chapter is divided as follows: The first 
sub-section will provide background information on relevant topics. Then, the problem statement will be exposed followed by the research objectives and the proposed methodology. Finally, the main contributions will be outlined and an overview of the remaining parts of the thesis will be given.

\section{$1.1 \quad$ Background}

In this part, we first review the anatomy of hip joint followed by information about FAI. Then, the concept of muscle contraction based on muscle physiology will be discussed. Finally, we express the methods to detect EMG signal from the muscles.

\subsubsection{Anatomy of the Hip Joint}

The structure of hip can be divided into four categories as follow:

- Bones and joints: The hip bones are the femur and the pelvis as shown in Figure 1.1. The top structure of the femur is like a ball and it is called the femoral head. The femoral head fits into the acetabulum which is a round socket. Thus, the hip joint is a ball (femoral head) and socket (acetabulum which is a part of the pelvic bone) joint to support the standing, walking, and running posture of the body. The femoral head and the surface of the acetabulum are covered by articular cartilage which creates a smooth, low friction surface. Moreover, the articular cartilage enables the femoral head and acetabulum slither easily [3]. The hip joint has three degree of freedom motions which means it moves in the three various planes including sagittal (flexion/extension), frontal (abduction/adduction) and transverse (external/internal rotation) planes. Therefore, 
a wide range of movement depends on the hip joint.

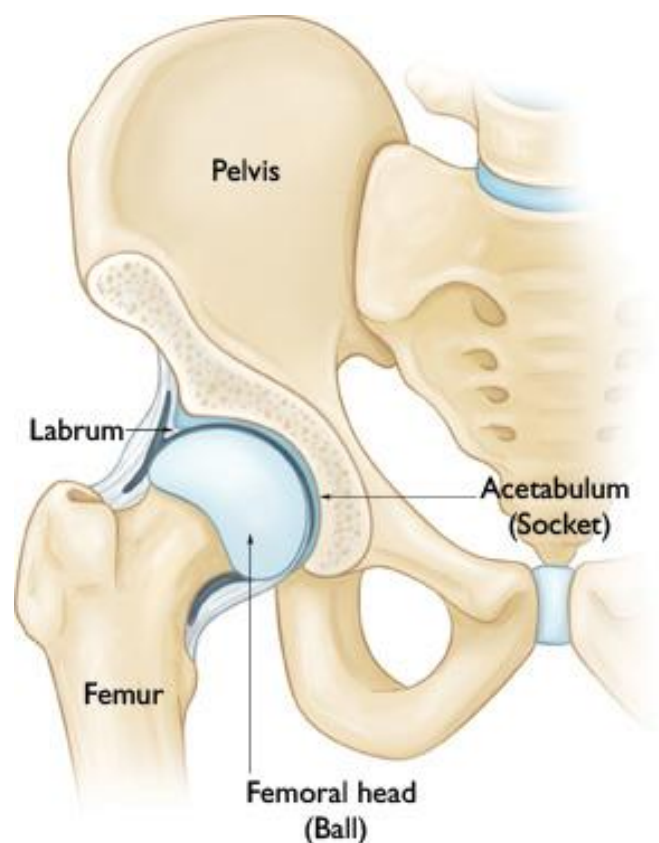

Figure 1.1: A Healthy Hip, Femoral Head Fits into the Acetabulum [1]

- Ligaments and tendons: Three major ligaments connect bones to other bones and prevent excessive movement and dislocation, stabilize the hip joint. The iliofemoral ligaments is the strongest capsular ligament which tightens on hip extension and external rotation of the hip [11]. The pubofemoral ligament tightens in hip extension and abduction and controls external rotation. The ischiofemoral ligament tightens on hip extension and restricting internal rotation [12].

- Nerves: The hip consists of two main nerves; the femoral nerve in front and the sciatic nerve in back. Also, there are many small nerves called obturator nerves. The function of these nerves is to carry the signals from brain to muscles 
(move the hip) also carry signals back to brain from muscles.

- Muscles: Hip muscles are divided into four different groups according to their position named gluteal, lateral, adductor, and iliopsoas that control the movement of the hip. Figure 1.2 illustrates the most significant muscles in hip area.
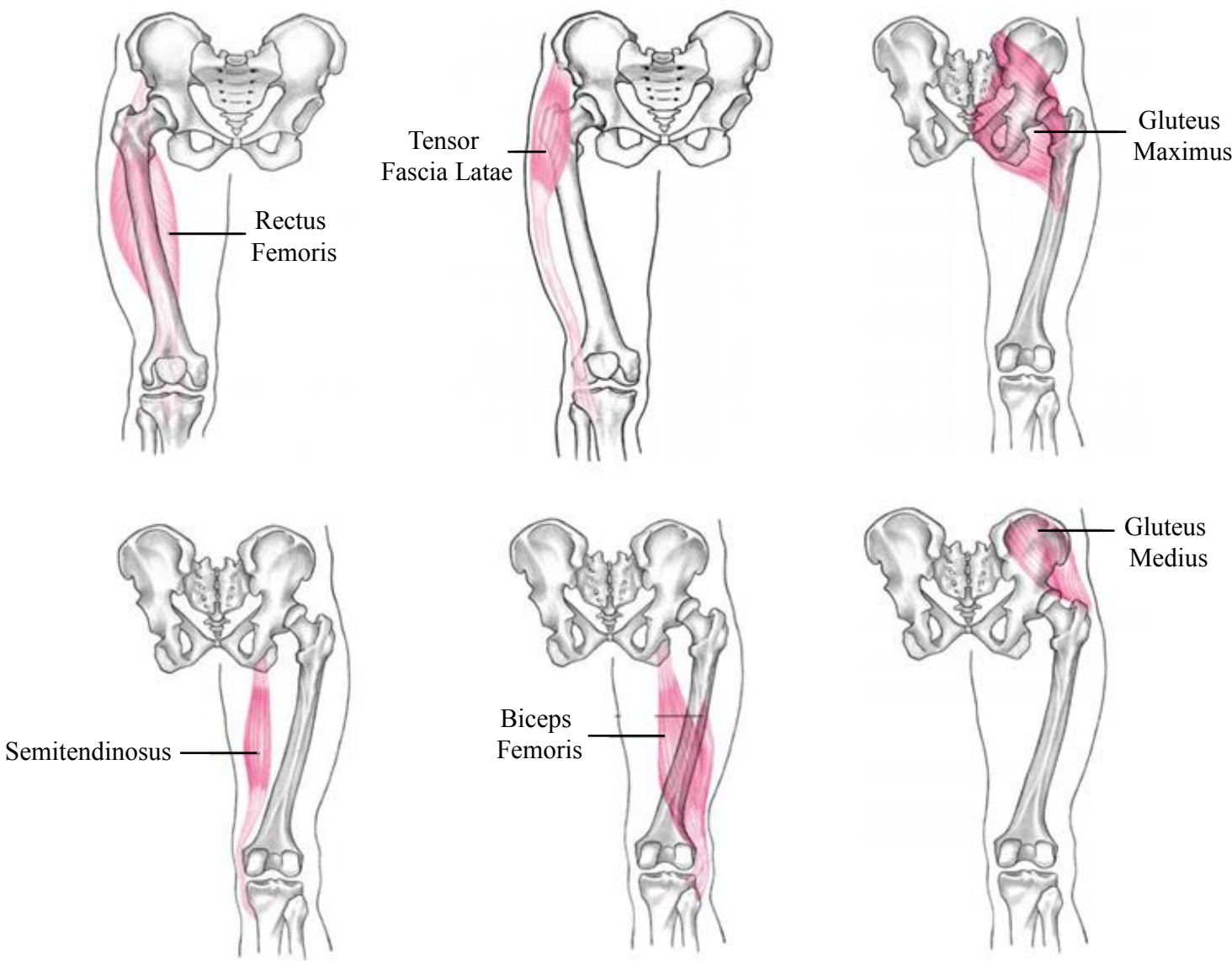

Figure 1.2: Muscles in Hip Area [2] 


\subsubsection{Femoroacetabular impingement}

FAI is a pathologic condition in which the femoral head and acetabular socket rub abnormally in the hip joint [13]. This abnormality reduces range of movements and performance in patients [3]. FAI usually appears in young and active adults with pain in groin area, although many FAI patients have no history of hip disease [7]. The physical activity and exercise may aggravate the pain in FAI patients [3].

FAI has two common forms known as cam impingement and pincer impingement however, the combination of both types is more common. Figure 1.3 indicates the diagram of the normal hip (a), hip with cam impingement (b), hip with pincer impingement (c), and the combination of cam and pincer impingement (d). 
a) Normal

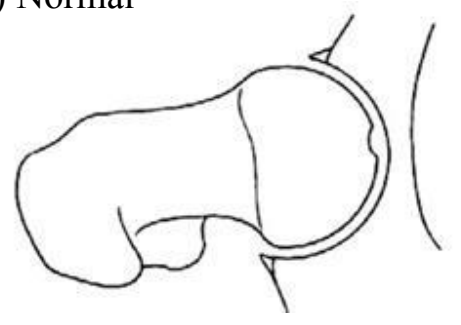

c) Pincer Impingement

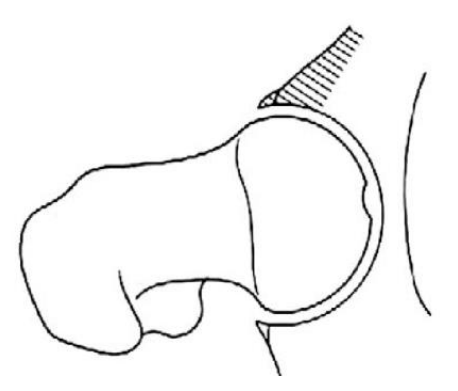

b) Cam Impingement

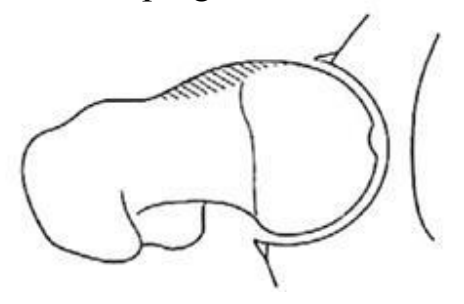

d) Combination of Cam and Pincer Impingement

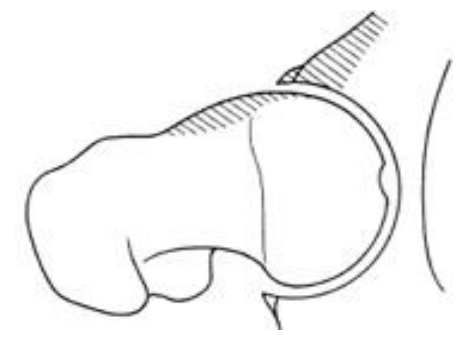

Figure 1.3: FAI Forms [3]

Cam Impingement, the femoral deformity, is an abnormality of the femoral headneck junction where the femoral head is not round and cannot rotate smoothly inside the acetabulum. Therefore, the cartilage inside the acetabulum is grinded. Pincer impingement, the deformity on the acetabular side, is the situation where abnormality of the acetabulum causes impingement whereas the femoral head is morphologically normal $[3,14]$. 


\subsubsection{Skeletal Muscle Physiology}

An overview of skeletal muscle structure is indicated in Figure 1.4.

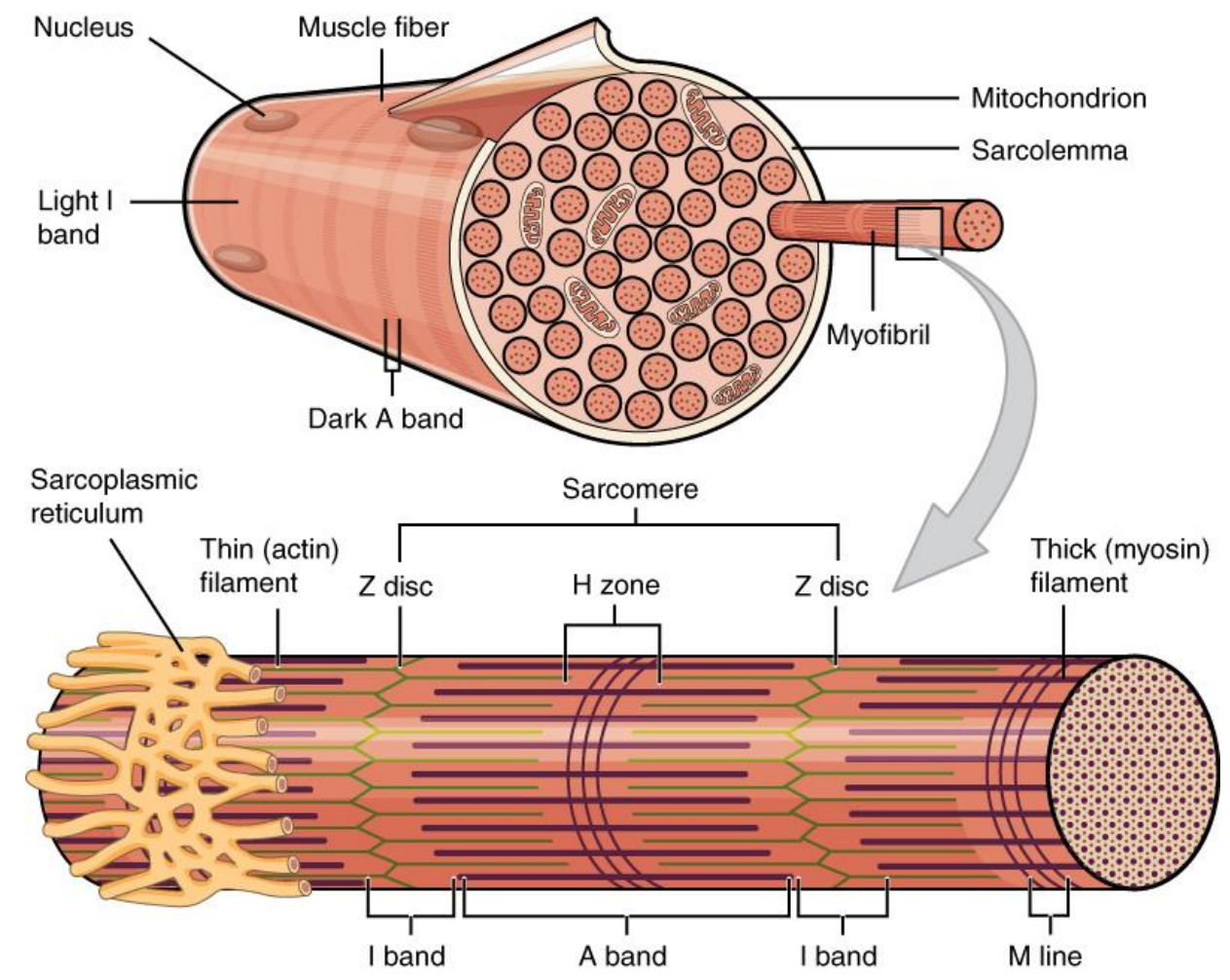

Figure 1.4: Overview of Skeletal Muscle Structure [4]

The most prominent constituent of the muscle fiber is myofibril which is responsible for causing muscle cell contraction. Myofibrils have a contractile unit called sarcomere. The internal structure of the myofibril is composed of thick filaments (myosin) and thin filaments (actin). The myosin filaments have cross-bridges that are able to attach temporarily to the actin filaments. The sarcomeres consist of sev- 
eral regions such as dark bands ( $A$ bands), and light bands ( $I$ band), and the ends of each sarcomere are defined by $Z$ disc. The $A$ and $I$ bands related to the present or absence of myosin filaments. This means the $I$ bands comprise only the actin filaments, whereas the $A$ bands consist of myosin filaments. At the middle of $A$ band there is a region called the $H$ zone which includes only the myosin, however in peripheral regions of $A$ band the myosin and actin overlap.

During muscle contraction, the actin filaments slide past the myosin filaments toward the middle of the sarcomere. In this situation, sarcomere shortens by the $Z$ discs become closer to one another. The region for $A$ band does not change, whereas both $I$ and $H$ zone almost disappear. Figure 1.5 indicates the sliding-filament model of muscle contraction [15]. 


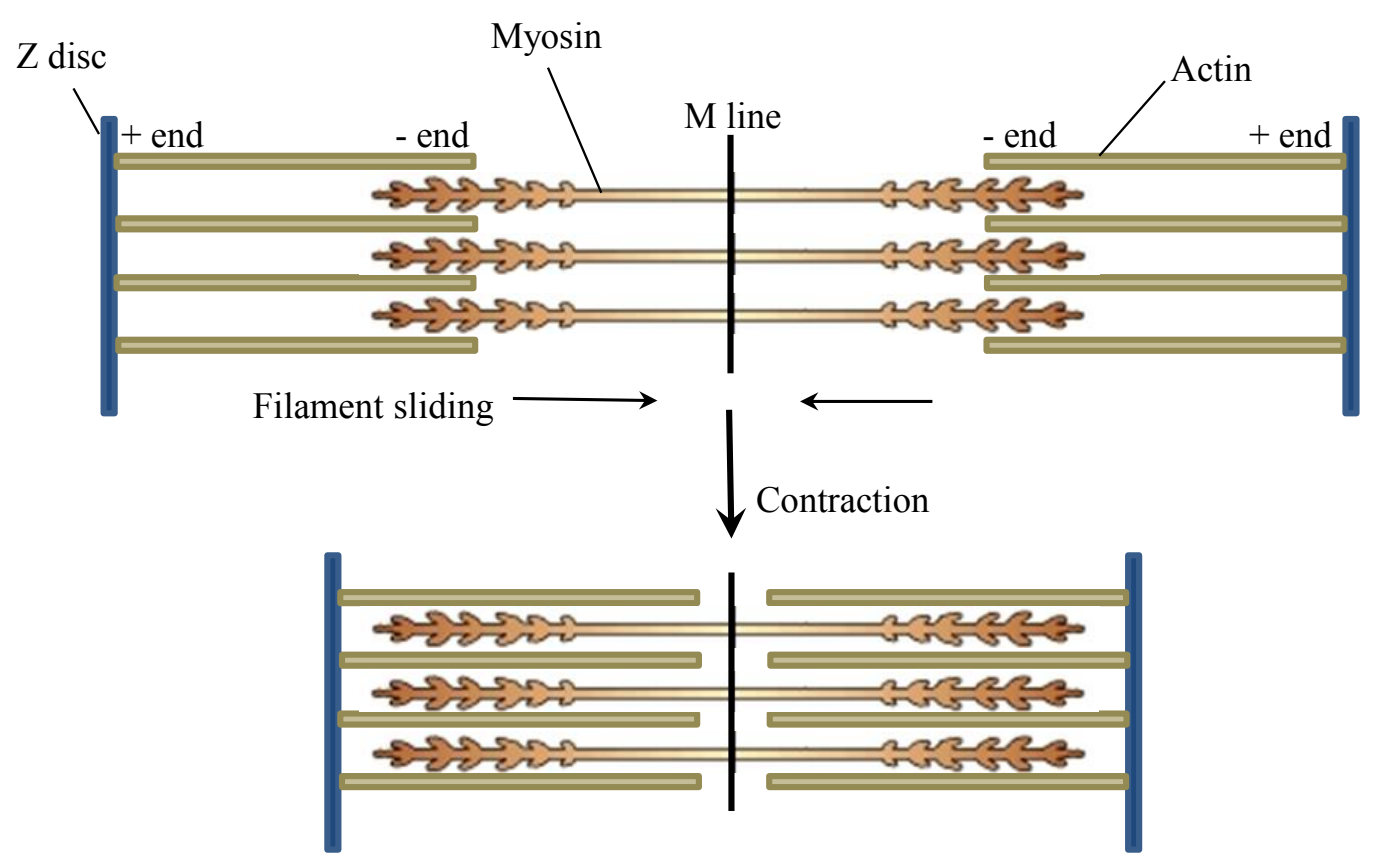

Figure 1.5: Sliding-Filament Model of Muscle Contraction

\subsubsection{EMG Signals}

The electrical activity in the muscles arises from the contraction of the muscle fibers. The EMG signal is the sequence of voltages recorded from a contracting muscle over time. The muscle signal called myoelectric (ME) signal that is detected via EMG by using electrodes. This signal is composed of the action potentials (AP) travelling across the muscle fibers and each of these APs occur at random intervals. The muscle fibers are innervated in groups by $\alpha$-motoneurons. Furthermore, a single $\alpha$-motoneuron and the muscle fibers it innervates called a Motor Unit (MU) [16]. Figure 1.6 shows the motor unit and its components [17]. 


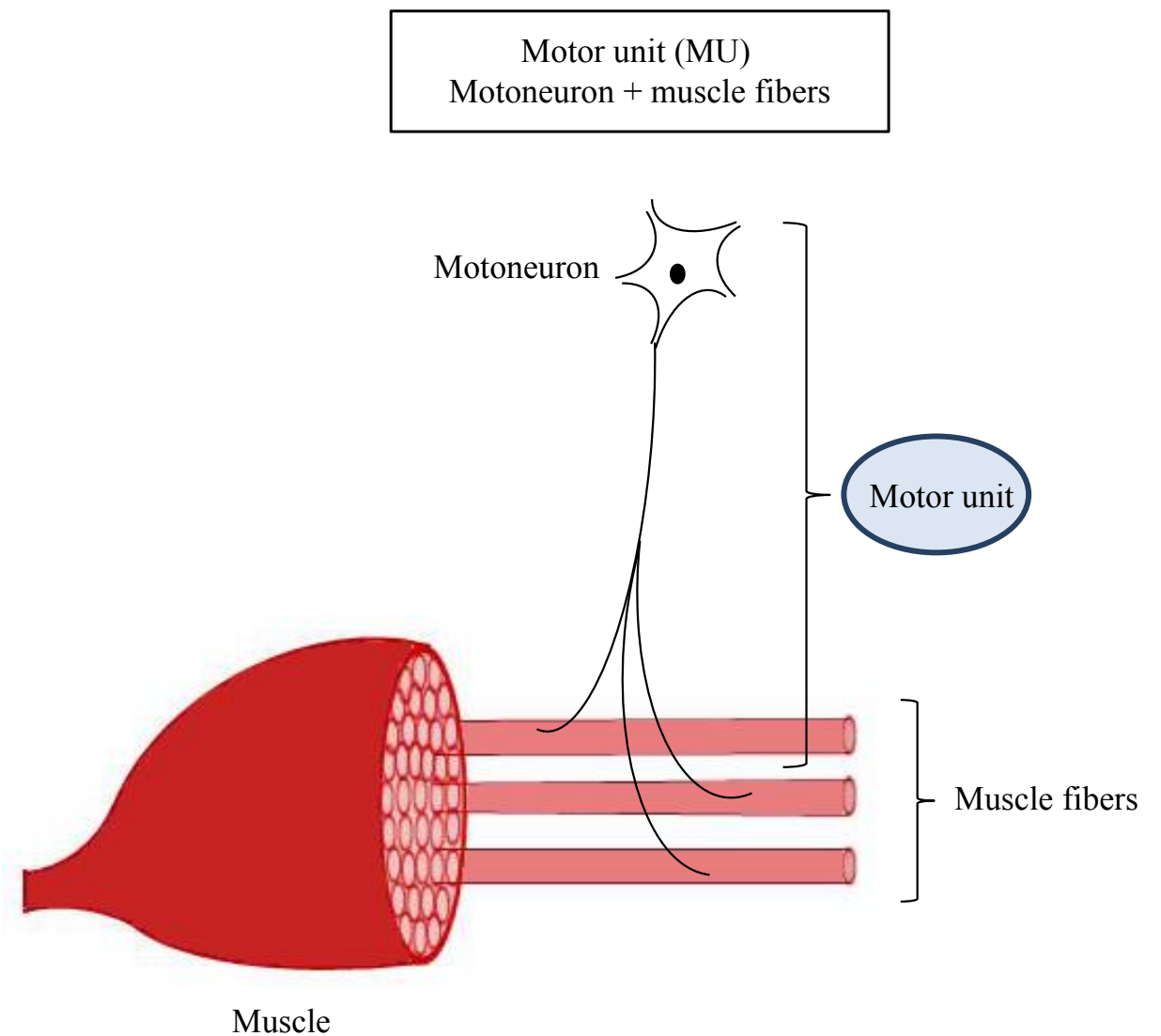

Figure 1.6: Motor Unit

MUs and the $\alpha$-motoneurons vary in size. Small motoneurons innervate relatively few muscle fibers, while large motoneurons innervate larger amount of fibers. MUs also differ in the types of muscle fibers that they innervate. Generally, the small MUs are called slow motor units (type I), innervate small muscle fibers that contract slowly and generate small forces. These slow MUs are important for activity that require sustained muscular contraction. The fast fatigable motor units (type IIB) are significant for brief exercise which require large forces. The fast fatigue-resistant motor units (type IIA) have intermediate size and are not quite as fast fatigable 
MUs. The fast fatigue-resistant MUs are substantially more resistant to fatigue and generate about twice the force than a slow motor units.

The motor unit action potential (MUAP) can be detected by invasive and noninvasive methods. In the invasive method, electrodes are inserted through muscle tissue (wire or needle electrode), whereas in the non-invasive method the electrodes are placed on the surface of the skin (surface electrode) on top of the muscle. The recorded signal called "raw EMG" has noise and it is normally a function of time.

The surface electromyography (sEMG) is a non-invasive technique that has potential usage in sports and rehabilitation medicine. Studying EMG signals can assess us to control the muscles performance in daily tasks and obtain information about the movement of the muscles.

\subsection{Problem Statement}

FAI is common in high level athletes, but also occurs in active individuals. FAI can be diagnosed using plain x-ray films to determine the shape of the femoral head and acetabulum as well as to indicate the amount of joint space in the hip. Magnetic resonance imaging (MRI) of the hip also can be used to confirm the damage to the joint. These methods are useful for diagnosing FAI based on bone deformation; however FAI patients may have abnormality in their hip muscles compared to a person without FAI.

Electrical activity of muscle provides significant information for physicians and doctors. The muscle activity can be measured between the FAI and healthy controls in order to assists physicians to diagnose FAI based on EMG activity of muscles. 
In this thesis, the goal is to detect FAI in people based on the analysis of their EMG signal. Most analysis methods proposed in literature deal with different muscles in the body; however none of the studies consider the hip muscles for FAI people. Thus, the overall aim of this project is to develop an algorithm to automatically distinguish between FAI and healthy controls based on analysis of sEMG signals from hip muscles.

The outcomes of this study are important in terms of diagnosis of FAI patients based on their muscle activities instead of just considering the bone deformation. Using electrical activity of muscles for diagnosis of FAI can be a complement to MRI and x-ray methods.

\subsection{Research Objectives}

The main objective of this thesis is to develop an algorithm based on recorded EMG signal from three different muscles in hip area in order to discriminate FAI patients from healthy subjects. More specifically, we aim to:

1. Compute the signal's linear envelope for amplitude analysis of the EMG

2. Calculate integrated EMG and discriminate between groups

3. Develop approximate algorithms based on computing energy of EMG signals using wavelet transform methods and select a wavelet function to distinguish FAI from healthy control

4. Develop wavelet power spectrum of EMG signals and compare the performance of the algorithm 


\subsection{Methodology}

The EMG signal is a complex signal which contains valuable information. Different signal processing methods are applied on raw EMG in order to attain the actual EMG signal. Discriminating the FAI and healthy control groups is an exhaustive task, as the variability between them is high and it is hard to differentiate the groups. The methodology used to approach the solution in this thesis is described below:

1. Using signal processing methods: Signal processing methods were used to obtain the actual EMG from the raw EMG.

2. Study wavelet transforms: In order to acquire a good knowledge about decomposition of EMG signals, the wavelet transform was studied and compared with other time-frequency methods.

3. Select a wavelet function for our EMG signals: Reviewing the literature on wavelet transform reveals that there is not a specific method to choose a wavelet function so far. In order to select a wavelet appropriate for our sEMG signal, various wavelet families are required to be considered.

4. Calculate the energy from various wavelet families: Applying various wavelet functions to EMG signals and selecting the one that gives the highest amount of energy for most of signals.

5. Implement algorithm: The wavelet transform method was used to solve the thesis problem. Due to high variability between FAI and control groups, we used MATLAB programming language to calculate the wavelet power spectrum. 
6. Discriminate FAI from healthy controls: The problem was solved by wavelet transform method. The power of signals were calculated and compared together. Finally, the efficiency of time-frequency methods to solve the thesis problem were investigated and analyzed.

\subsection{Contribution}

The main contribution of this thesis is summarized as follows:

1. Identification of Femoral-Acetabular Symptoms Using sEMG Signals During Dynamic Contraction: An algorithm for discriminating the FAI from healthy control was proposed and analyzed. As the wavelet transform represents a very suitable method for the classification of EMG signals, this time-frequency method was used in this algorithm. The wavelet analysis has an advantage over FFT which provides the frequency contents of the signal over the time period that is being analyzed. As the shape of the wavelet and MUAP is related to a better decomposition, wavelet analysis was chosen to match the shape of the MUAP, and the results yield the best possible energy localization in time-scale plane. In this algorithm we decomposed sEMG signal from Gluteus Maximus, Tensor Fasciae Latae, and Rectus Femoris muscles using discrete wavelet transform for different wavelet functions and calculated the energy during dynamic contraction in squat task. A wavelet function which provided the highest amount of energy in most of our subjects was selected. Furthermore, the wavelet power spectrum was computed and used for discriminating the FAI patients from the healthy controls. The results of this work will be submitted 
to a journal paper.

\subsection{Thesis Overview}

The remainder of the thesis is organized as follows. In Chapter 2, we present a selective review on muscle weakness in FAI patients and time-frequency analysis methods. In Chapter 3, we present the proposed methodology and algorithms used in this work. In Chapter 4, we present the simulation results and discriminate FAI and control groups. In Chapter 5, we conclude the work and suggest future directions. 


\section{Chapter 2}

\section{Literature Review}

\subsection{Background}

The EMG signal is a complicated signal which is controlled by nervous system. The anatomical and physiological properties of muscles have influence on the recording of the EMG signal. Moreover, the EMG signal can be affected by various external factors varying its shape and characteristics. Some of these factors are tissue feature, physiological cross talk, changes in the geometry between muscle belly and electrode site, external noise, and electrode and amplifiers [18].

An EMG signal recorded from muscles requires advanced methods for detection, decomposition, processing, and classification. In order to use the EMG signal as a diagnosis signal, a feature is extracted before analysis or classification of it. This is due to the fact that the raw EMG signal includes both useful information and noise. EMG features can be computed in various domains such as time, frequency, timefrequency, and time-scale domains. Analysis of EMG data requires rectification and 
integration of signals or root mean square values to extract information related to the amplitude of the signal which deals with the time domain representation. However, the frequency content of EMG is analyzed using Fourier Transform (FT) that is a uni-dimensional technique. Moreover, a signal can be stationary or non-stationary. If a signal is stationary, its properties are statistically invariant over time; however the transient events cannot be predicted in non-stationary signals. The EMG signal is a non-stationary signal, thus FT and other classical signal processing methods are not suitable for analyzing EMG signals recorded during dynamic conditions. Furthermore, time-frequency methods that are appropriate for non-stationary signal are used instead of frequency methods to improve the EMG analysis [19]. Thus, for analyzing EMG signals in both time and frequency, the short-term Fourier transforms (STFT) and wavelets can be accomplished. The processing of the EMG signals usually has three steps: recording the EMG signals, decomposition of signals by signal processing techniques, and classification of signals for diagnostic purpose.

In this chapter, first a literature review in the area of muscle weakness in FAI patients is presented. Then, time-frequency analysis method is discussed in detail. Finally, the best method in analyzing the EMG signals is presented as well as the related works in literature.

\subsection{Muscle weakness in FAI}

FAI is a pathomechanical process which causes hip pain and disability in people. It is

the deformity of bones that leads to joint damage and pain. FAI patients experience functional disability during dynamic activities that can be related to the weakness 
of the hip muscles. Although there are not many studies on muscle weakness in FAI patients, the few existing studies demonstrated the relationship of hip muscles. Casartelli et al. [20] attempted to compare hip muscle strength and hip flexor electromyography activity between symptomatic FAI patients and healthy controls. They stated the overall hip muscle weakness in symptomatic FAI patients. Isometric maximum voluntary contraction (MVC) strength of the hip muscles in three planes (sagittal, frontal, and transverse) was measured unilaterally. Muscle weakness was quantified by comparing strength outcomes between FAI patients and healthy controls. Hip abduction, adduction, internal rotation, and external rotation were randomly tested first. Then, the hip flexion and extension were determined in a randomize order. Hip abduction, adduction, internal and external rotation MVC strength evaluated with hand-held dynamometry, is a portable device which measures strength during manual muscle testing. In addition, hip flexion and extension MVC strength determined with isokinetic dynamometry in order to concurrently record MVC torque and EMG traces. Also, in a subgroup, the muscle activity of two main hip flexor muscles including the Rectus Femoris (RF) and Tensor Fasciae Latae (TFL) was measured by using EMG during active flexion. The highest MVC was considered in each muscle groups and for better distinguishing muscle weakness, the percentage difference in MVC strength was calculated. Casartelli et al. also recorded the EMG signal from RF and TFL muscles during muscles contraction. In general, they affirmed that the MVC was significantly lower in symptomatic FAI patients compared to healthy controls for hip adduction, flexion, external rotation, and abduction. Moreover, FAI patients presented lower EMG activity in TFL muscles than healthy controls. Furthermore, one year after their first paper, Casartelli et al. [21] expressed that the symptomatic FAI 
patients have hip muscle weakness especially in hip flexion and hip adduction. They postulated in alignment to hip flexor muscle weakness, FAI patients have greater hip flexor fatigue than healthy controls. For considering the hip flexor fatigue, they performed two experiments. In the first experiment, the hip flexor torque fluctuation and EMG activity were recorded simultaneously during continued submaximal isometric contraction for RF and TFL muscles. In order to evaluate fatigue, the average hip flexion torque fluctuation, average EMG root mean square (RMS) and median frequency (MDF) were calculated. In the second experiment, hip flexor torque decline was assessed during a maximal dynamic contraction. Then, the weight-normalized isokinetic torque and the rate of torque decline were calculated. As a result, Casartelli and his collages proved that under isometric and isokinetic situation FAI indicates hip flexor weakness. In addition, Mantovani et al. [22] analyzed a group of hip muscles during squat in order to calculate the co-activation index (higher co-activation index causes stiffness in the joint and enhance joint contact loading) for FAI patients and compared that to healthy controls. They believed that the symptomatic FAI patients had higher muscle co-activation levels compared to asymptomatic FAI and controls. They considered three groups of unilateral symptomatic FAI, unilateral asymptomatic FAI, and control. The contribution of a group of muscles including Gluteus Maximus (GMax), Biceps Femoris (BF), Semitendinosus (ST), TFL, and RF muscles were evaluated by recording EMG activity based on SENIAM guidelines. The co-activation index (CI) which showed the contribution of a group of muscles was estimated during descending and ascending phase for both affected and unaffected sides. They concluded that the high muscular co-activations were indicated in the affected side for the asymptomatic FAI group. 
In general, the muscle weakness in FAI can be obtained by considering the activity of each hip muscle separately or by evaluating the interaction between hip muscles.

\subsection{Time-Frequency Analysis Methods}

Traditional techniques for analyzing surface EMG signal are based on the FT method. The accuracy and reliability of this technique depends on the data lengths' and the signal requires to be stationary. In daily life activities or movements, subjects perform more dynamic contractions instead of isometric contractions. Moreover, in the field of rehabilitation medicine, sports medicine, and etc., tasks similar to daily activities are performed. During dynamic conditions, the Fourier and other classical signal processing methods are not suitable for analyzing the myoelectric (ME) signals as a number of active motor units, active muscle fibers, electrode geometry, muscle fiber lengths, and innervation zone geometry changes. These factors emphasize that the ME signals are non-stationary. Therefore, time-frequency methods have been introduced for the analysis of non-stationary signals. These time-frequency representation methods are the STFT, Wigner-Ville distribution (WVD), Choi-Williams distribution (CWD), and wavelet transform (WT) that were compared with recent studies for accuracy and precision to analyze the ME signals [19,23].

Gabor [24] developed the STFT method based on Fourier transform method. The input signal was divided into segments and the signals in each window were stationary. The STFT analyzed the signal with fixed resolution and mapped the distribution of the energy of the signal in the time-frequency plane which was called spectrogram [5].

WVD can be used as a time-frequency energy distribution method which intro- 
duced by Wigner and then modified by Ville in order to be applied to the signal processing field [25]. Duchne et al. [26] used WVD method for analyzing uterine EMG in order to track instantaneous burst frequency. In this method the instantaneous frequency was equivalent to the mean frequency. The result delivered by Duchne et al. confirmed that converting the ME signals to the time-frequency plane by applying WVD method to the ME signals was approving the relationship between sEMG and electrical activity of the uterine muscle at a cellular level. Furthermore, Knaflitz et al. [27] used Wigner-Ville transform to evaluate muscle fatigue during dynamic contractions. The instantaneous mean frequency was reduced and this phenomenon caused the muscle fatigue. The reduction in frequency was related to the decrease in velocity of depolarization of propagation along the muscle fibers. The result was a low time-resolution associated to body movement. Thus, the Wigner-Ville transform was not a suitable method to deal with multi component signals.

Bonato et al. [28] analyzed the ME signals during dynamic contractions. They compared different time-frequency methods such as the WVD, reduced interference, cone kernel and CWD by using different types of kernels. As a result, they proposed that the CWD was the most appropriate time-frequency method for analyzing the non-stationary ME signals in a dynamic contraction.

A time-frequency analysis based on wavelets [29] which is introduced recently is an appropriate tool to overcome the limitations of the traditional time-frequency methods. Karlsson (2000 and 2001), introduced the wavelet transform as a "mathematical microscope"that help observe various parts of the signal by setting the focus $[19,23]$. The WT has some advantages over the other time-frequency methods. As represented in Figure 2.1, the WT uses short window for high frequencies and long 
window for low frequencies, although the STFT uses a single analysis window for all frequencies [5]. Furthermore, the WT can be used to analyze both stationary and

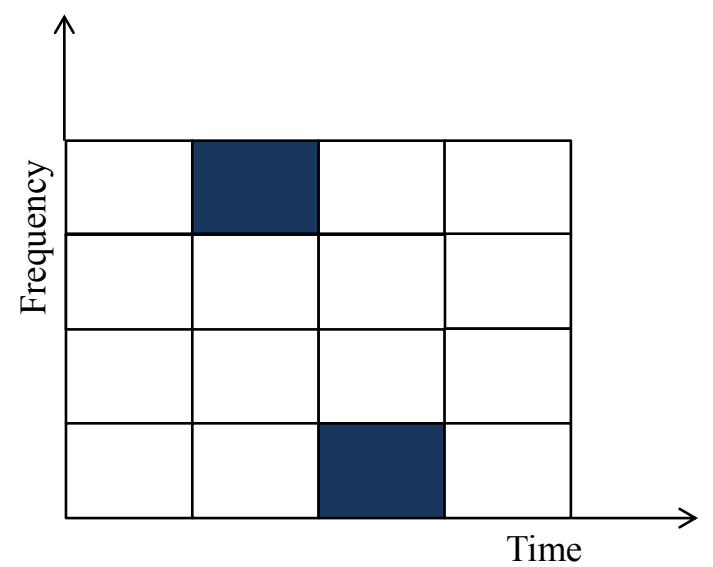

STFT

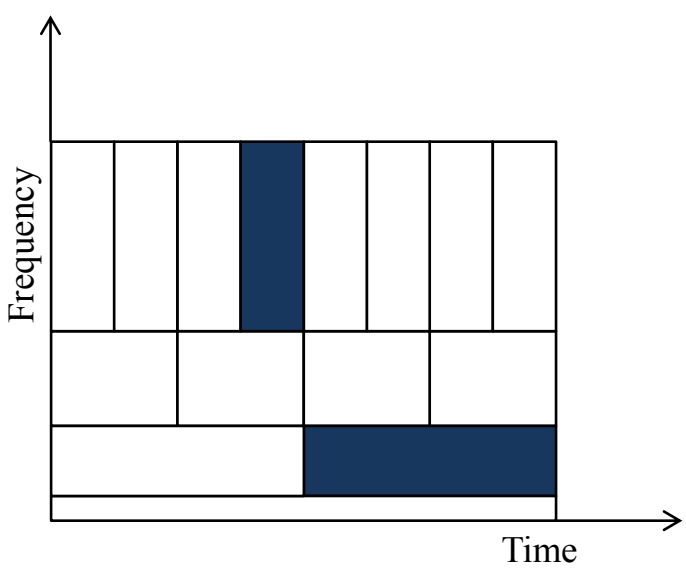

Wavelet

Figure 2.1: Time-Frequency Plane in STFT and WT [5]

WT method recently introduced overcome other time-frequency methods due to high performance and advantages. The wavelet theory covers both the continuous and discrete time cases. In the following sub-sections, wavelet transforms will be explained. The continuous and discrete WT will be introduced and their specifications will be discussed. Wavelet types and families will be presented following by choosing a wavelet for analyzing EMG signals. Also, different studies that have used WT to analyze EMG signals are explained. 


\subsubsection{Wavelet Transforms}

Historically, the concept of wavelets developed mostly in the early 1980s. This new concept is connected to many fields including mathematics, physics, computer science, and engineering. The history of mathematics leading to Wavelets began with Joseph Fourier (1807) with the theory of frequency analysis. He believed that functions can be represented as the summation of sines and cosines. Also, Joseph delivered the idea of Fourier Transform, which transforms a function in time domain into a new function in frequency domain. The first presentation of wavelets appeared by Alfred Haar in 1909. The Haar wavelets are the simplest of the wavelet families and are not continuously differentiable which limits their applications. Jean Morlet around the 1975 used the term wavelet to define his functions. In 1982, Morlet discovered the idea of wavelet transform. He believed that keeping the window fixed when using windowed Fourier analysis was not an appropriate approach. Thus, Morlet changed the window while the frequency of the function was kept constant. Based on his finding, stretching the window stretched the function and squeezing the window compressed the function. In late 1986, Yves Meyer and Stephane Mallat discovered the multi-resolution analysis for wavelets. In 1988, Ingrid Daubechies used the multi-

resolution analysis idea in order to produce the Daubechies families of wavelets [30]. The Daubechies wavelets have compact support, orthogonality (the inner products of all of the different translates are zero), regularity (can produce linear functions), and continuity.

Wavelets are functions that oscillate above and below the $\mathrm{x}$-axis and have varying frequency, limited duration, and an average value of zero [31]. Wavelets are localized 
in time-frequency space and wavelet transformation has been used for the processing of signals which are non-stationary and time varying. Each wavelet is represented by a bell shaped filter curve which has a center frequency at the point of maximal amplitude and a mode parameter that controls the bandwidth of the wavelet. The WT is achieved by a convolution of wavelets with the EMG signal. This convolution can be performed in time or frequency space. Wavelets have an automatic adapt window in order to give the appropriate resolution. The size of this window is not fixed and varies with frequencies; short/narrow window for high frequencies and long/wider window for low frequencies [19,32]. Therefore, wavelets are used to focus on short time intervals for high frequency components and longtime intervals for low frequency components and this property improves the signal analysis. A new approach for the analysis of EMG signals was developed by von Tscharner [31] using a filter-bank of non-linearly scaled wavelets. According to von Tscharner (2000), wavelets can be represented in time or frequency space for the intensity analysis. Thus, wavelets as functions of frequency are defined by center frequency and scale parameters. Tscharner described a time-frequency analysis of the intensities in time series. He used a filter bank of non-linearly scaled wavelets for this purpose. The intensity was calculated in order to approximate the power of the signal in time. The result of his research was the intensity patterns extracted from the EMG signal. Tscharner stated that the wavelet analysis method had an advantage over analyzing EMG by root mean square and averaging rectified signals. By using wavelet analysis method, the intensity analysis did not eliminate the higher frequency components compared to two other methods. Furthermore, in wavelet space the intensity indicates all power aspects of the signal and its variation in time and frequency. Based on 
Barandum et al. [33], the demonstration of the intensity versus center frequencies of the wavelets provided a wavelet spectrum. Also, an intensity pattern which was called a scalogram was represented by three dimensional graph of the intensity versus time and center frequency. Rioul et al. [5] also stated that by applying wavelets the signal is mapped into a time-scale plane, which is equivalent to a time-frequency plane in STFT. This time-scale plane is called scalogram, which indicated the distribution of the energy of the signal.

The WT is classified into continuous wavelet transforms (CWT) and discrete wavelet transforms (DWT). CWT is used for signal analysis and operates over every possible scale and translation, whereas DWT is used for data compression and uses a specific scale and translation values.

\subsubsection{Continuous Wavelet Transform}

In wavelet analysis, the wavelet family consists of enlarged or compressed version of the basic function as well as translation. When the input signal is $\mathrm{x}(\mathrm{t})$, the equation for continuous wavelet transform (CWT) is as follows:

$$
C W T(a, b)=\int_{-\infty}^{+\infty} x(t) \frac{1}{\sqrt{|a|}} \varphi *\left(\frac{t-b}{a}\right) d t
$$

where $a$ is the scale parameter, $b$ is the translation or time shifting parameter, and $\phi$ is a basis function or wavelet function. The $*$ donates complex conjugation and the factor $\frac{1}{\sqrt{|a|}}$ is introduced for energy normalization $[5,32,34]$.

The wavelet coefficients, $\operatorname{CWT}(\mathrm{a}, \mathrm{b})$, indicate the correlation between signal and the basis function at different translations and scales. With a large $a$, when the basis 
function is stretched, the low frequency components of the signal can be analyzed. With a small $a$, when the basis function is contracted, the high frequency components of the signal can be analyzed.

The CWT generates more coefficients than are actually required to specify the signal. In other words, it provides an oversampling of the original signal. For analysis applications, this oversampling is not a serious problem; however the redundancy will be costly if the original signal needs to be recovered. Therefore, when the recovery of the original signal is significant, the discrete wavelet transform (DWT) is used to restrict the variation in translation and scale [34].

\subsubsection{Discrete Wavelet Transform}

The DWT is introduced in terms of its recovery transform through the following equation:

$$
x(t)=\sum_{k=-\infty}^{\infty} \sum_{l=-\infty}^{\infty} d(k, l) 2^{\frac{-k}{2}} \varphi\left(2^{-k} t-l\right)
$$

where $k$ is related to $a$ by $\left(a=2^{k}\right)$, and $b$ is related to $l$ by $\left(b=\left(2^{k} l\right)\right.$. Also, $d(k, l)$ is a sampling of $C W T(a, b)$. The DWT has the scaling function which is defined as the following equation:

$$
\Phi(t)=\sum_{n=-\infty}^{\infty} \sqrt{2} c(n) \Phi(2 t-n)
$$

where $c(n)$ is a series of scalars which relates to specific scaling function. The wavelet can be defined based on the scaling function as the following equation: 


$$
\varphi(t)=\sum_{n=-\infty}^{\infty} \sqrt{2} d(n) \Phi(2 t-n)
$$

where $d(n)$ is a series of scalars which is associated with $x(t)$ from equation 2.2. Thus, the DWT can be implemented using equation 2.4 based on filter bank technique $[34]$.

A very useful implementation of DWT called multi-resolution analysis is indicated in Figure 2.2. The original sampled signal $x(n)$ is passed through high-pass and lowpass filters that are shown by $h(n)$ and $l(n)$, respectively. The output of both high and low pass filters are decimated by 2 to achieve the detail $\left(D_{1}\right)$ and approximation $\left(A_{1}\right)$ coefficients. The approximation coefficients are passed through the second step in order to repeat the process. Therefore, the signal is decomposed according to the required levels. In Figure 2.2, the signal is decomposed at 3 levels [5].

Level $1 \quad$ Level $2 \quad$ Level 3

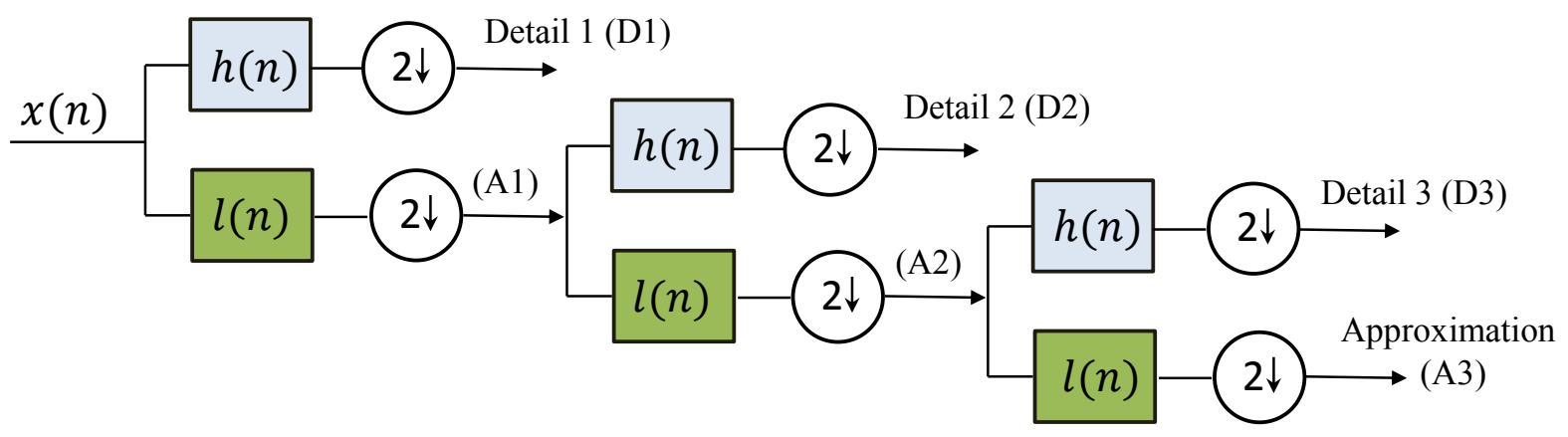

Figure 2.2: Wavelet Multi-Resolution Analysis [5]

During a typical wavelet analysis, wavelets of various lengths are applied to a 
particular signal. Then, the portion of the signal which is correlated with the shortest length (D1 which is first detail) is subtracted away. This means that the high frequency components which are representing the noise are removed. According to Figure 2.2, the filtering process splits the signal into high frequency (detail, D) and low frequency (approximation, A) bands [35]. The signal is downsampled in order to avoid doubling the number of data points at each level.

\subsubsection{Wavelet Types and Families}

There are different types of continuous and discrete wavelets available. The wavelet transform requires the selection of a mother wavelet depending on the application.

\subsubsection{Properties of Wavelet Functions}

Wavelets are defined by the scaling function (also called father wavelet) and wavelet function (or the mother wavelet). The scale function in WT determines wavelet dilation and compression of the various wavelets from a mother wavelet. Furthermore, the optimization of the WT is related to the scale function which is used for a particular signal. In the following, each wavelet families are introduced by representing their scaling and wavelet functions.

- Haar: Haar is the simplest wavelet which is discontinuous and resembles a step function. It illustrates the same wavelet as Daubechies db1. Figure 2.3 shows the scaling and wavelet function of this wavelet. 

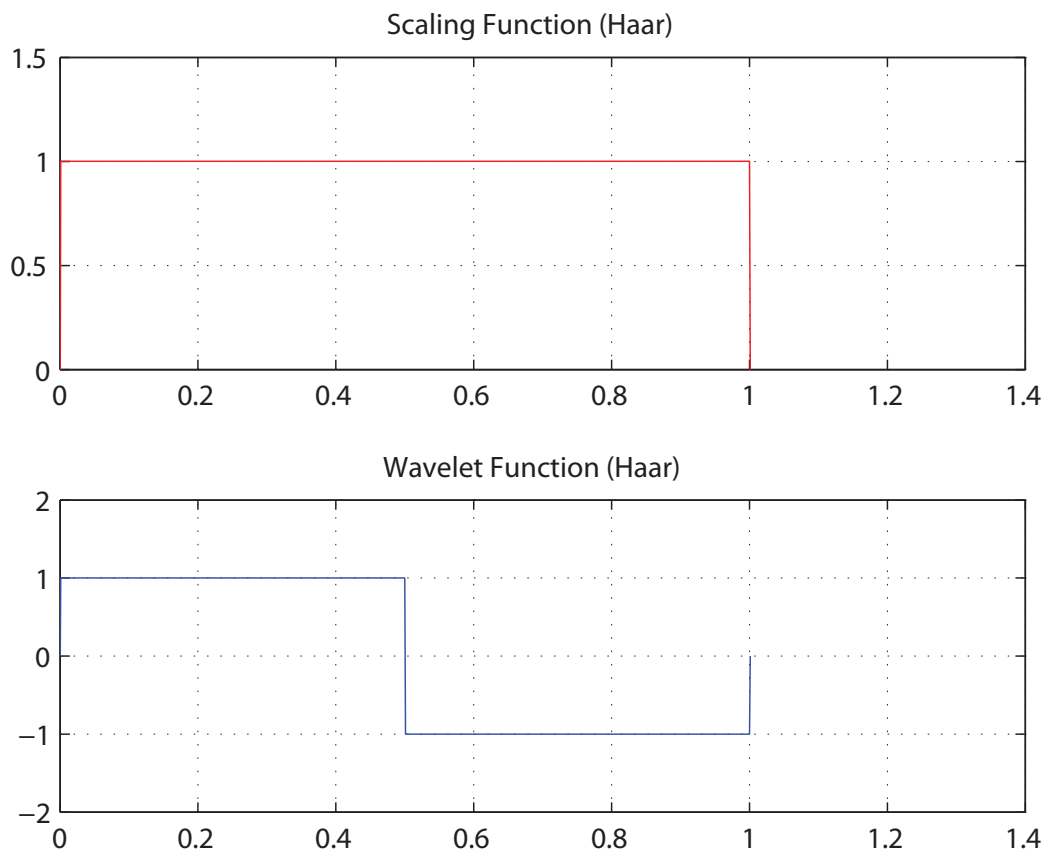

Figure 2.3: Haar Scaling and Wavelet Function

- Daubechies: Daubechies are compactly supported orthogonal wavelets. This family has highest number of vanishing moments which is indicated by $\mathrm{N}$ and has an external phase. Figure 2.4 shows the db2 and db10 scaling and wavelet functions when using MATLAB software. 

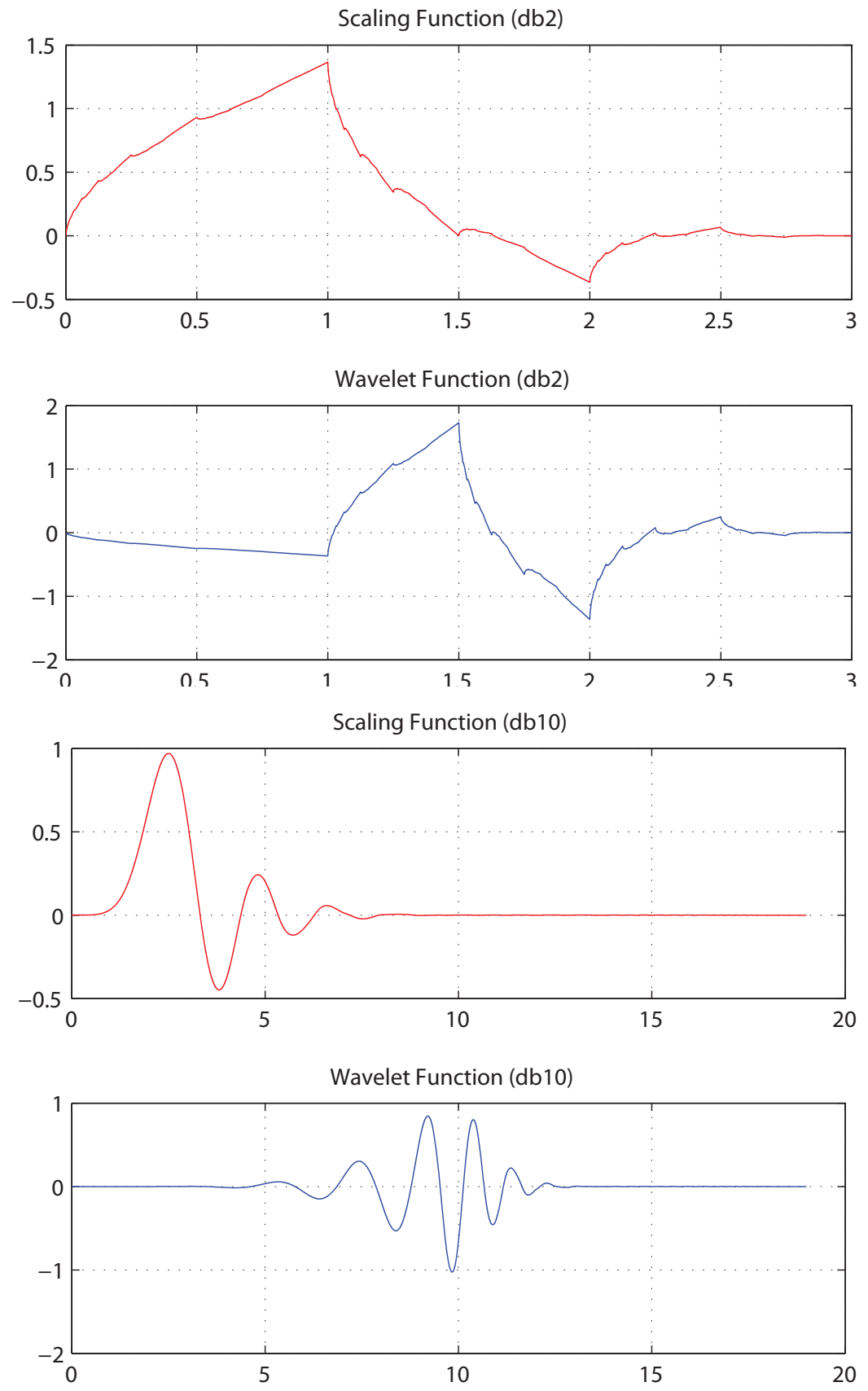

Figure 2.4: Daubechies 2 and 10 Scaling and Wavelet Functions 
- Symlets: Symlets family are compactly supported wavelets with nearly symmetric and highest vanishing moments similar to Daubechies family. This family is a modification of db family and with similar properties. Figure 2.5 exhibits the scaling and wavelet functions of symlets 8 .
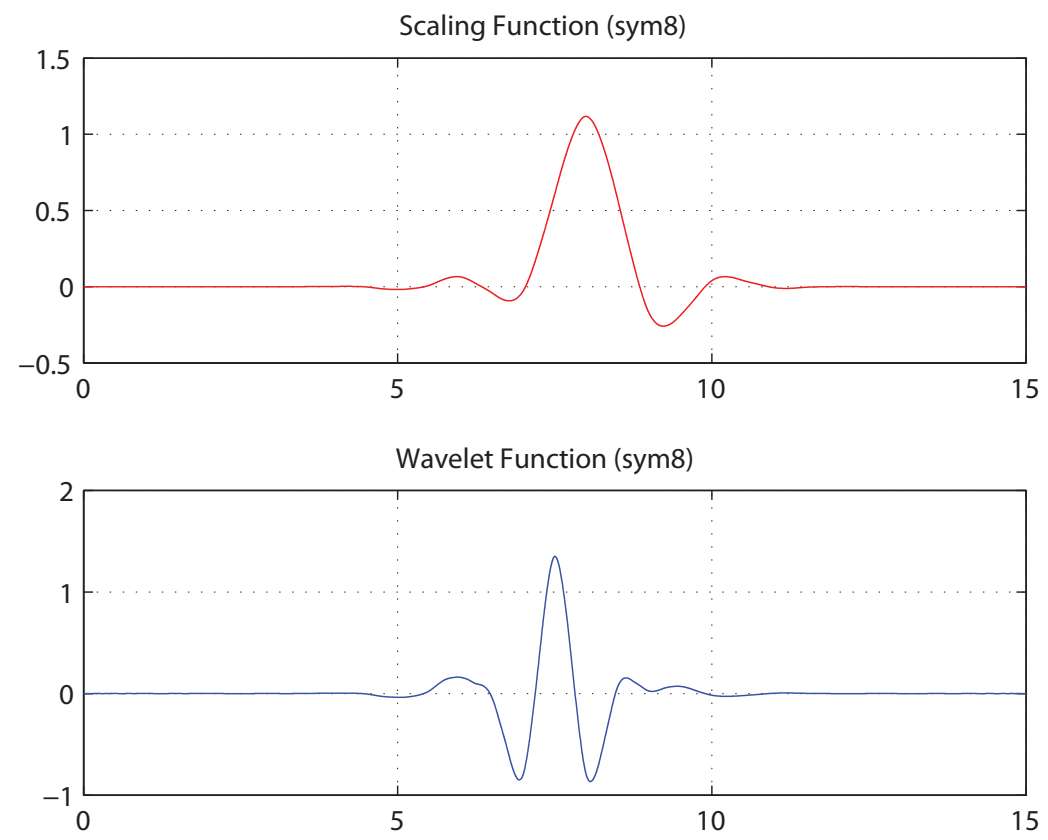

Figure 2.5: Symlets 8 Scaling and Wavelet Functions

- Coiflets: Coiflets family has the scaling function of $2 \mathrm{~N}-1$ moments equal to 0 and the wavelet function of $2 \mathrm{~N}$ moments equal to 0 . Figure 2.6 shows coif 2 and coif5 scaling and wavelet functions. 

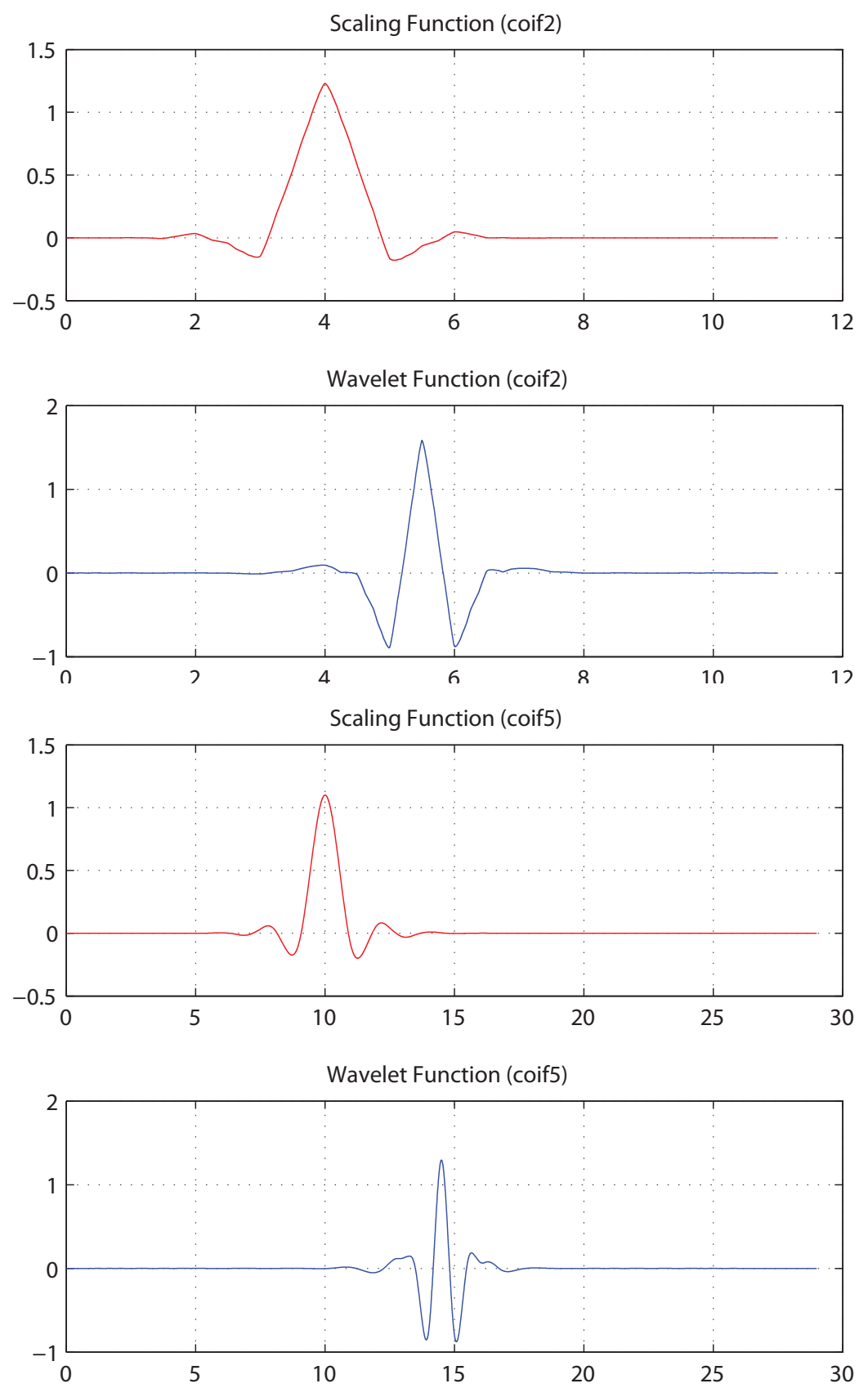

Figure 2.6: Coiflets 2 and 5 Scaling and Wavelet Functions 
- Biorthogonal: Biorthogonal family of wavelets illustrates the property of linear phase and includes two wavelets instead of one compare to other families; one for decomposition and the other one for reconstruction. Figure 2.7 illustrates the decomposition wavelet function which is significant in this research.

Decomposition Scaling Function (bior3.9)

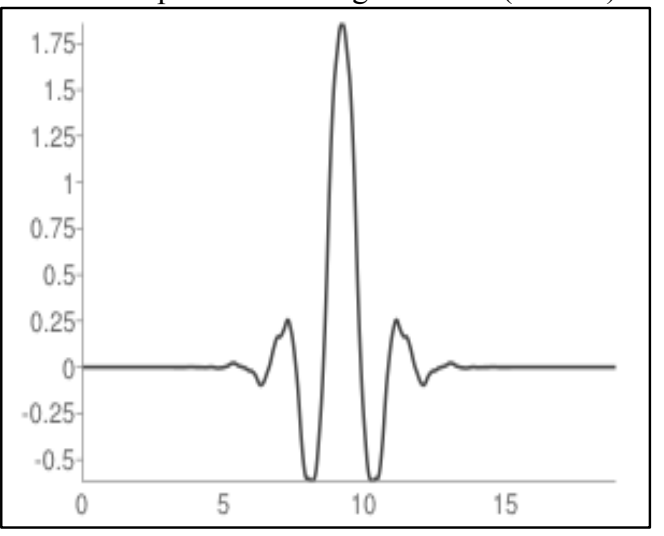

Decomposition Wavelet Function (bior3.9)

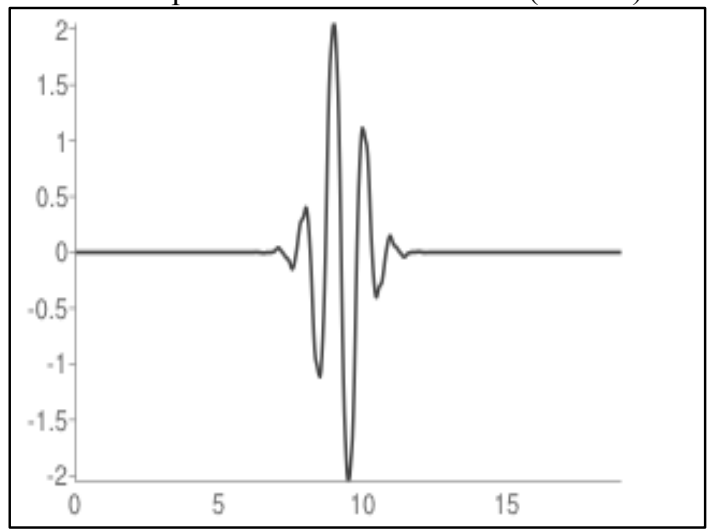

Figure 2.7: Biorthogonal Decomposition Scaling and Wavelet Functions

- Morlet: Morlet wavelet that is illustrated in Figure 2.8 has no scaling function. 


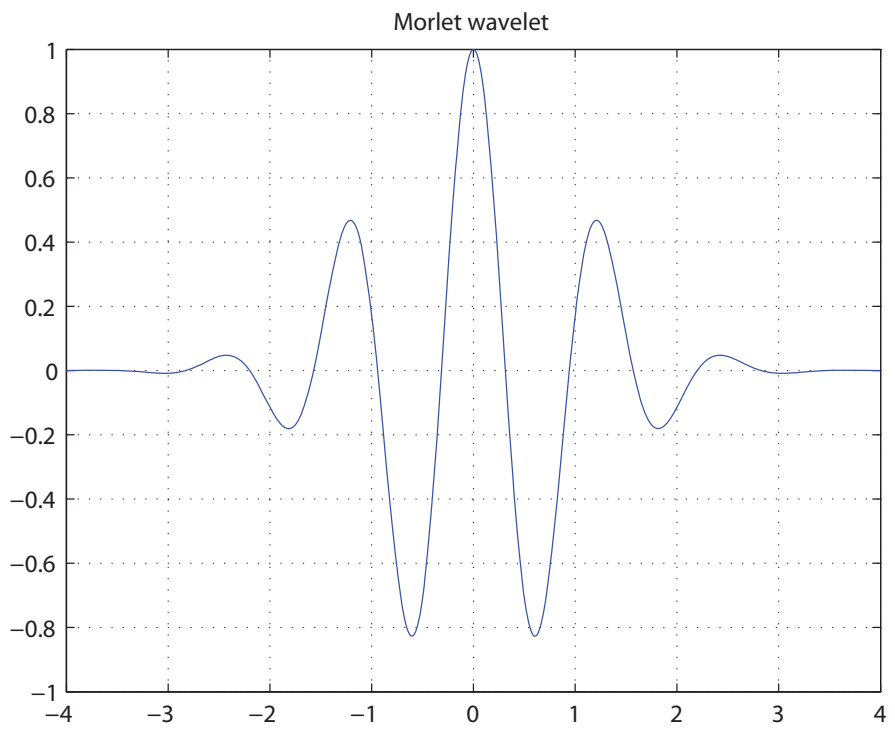

Figure 2.8: Morlet Wavelet

- Mexican Hat: This wavelet has no scaling function similar to Morlet wavelet. Mexican Hat wavelet function is represented in Figure 2.9.

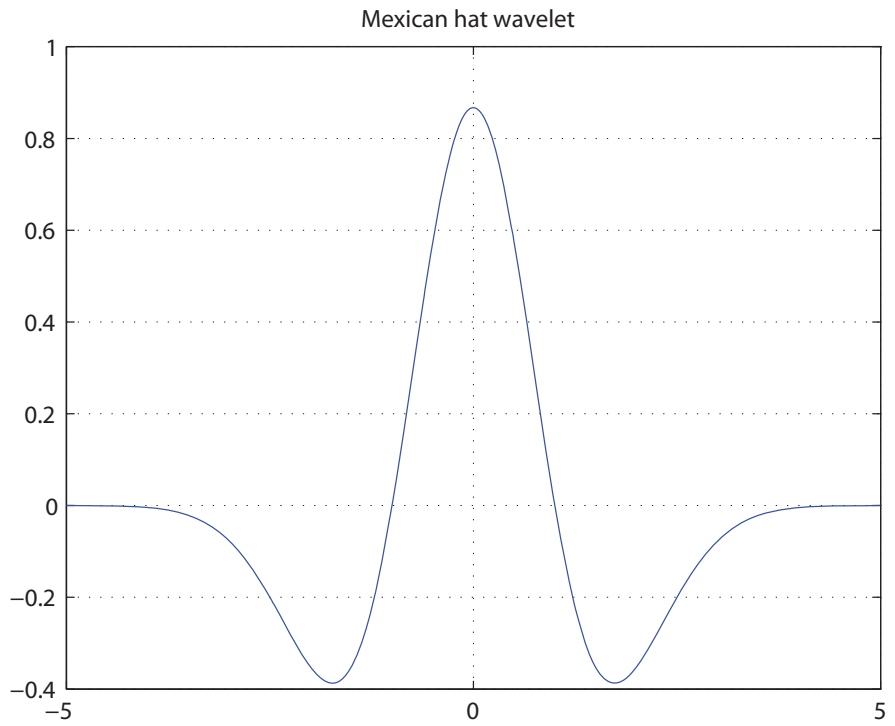

Figure 2.9: Mexican Hat Wavelet 
- Meyer: Meyer scaling and wavelet function is defined in the frequency domain. This wavelet is represented in Figure 2.10.
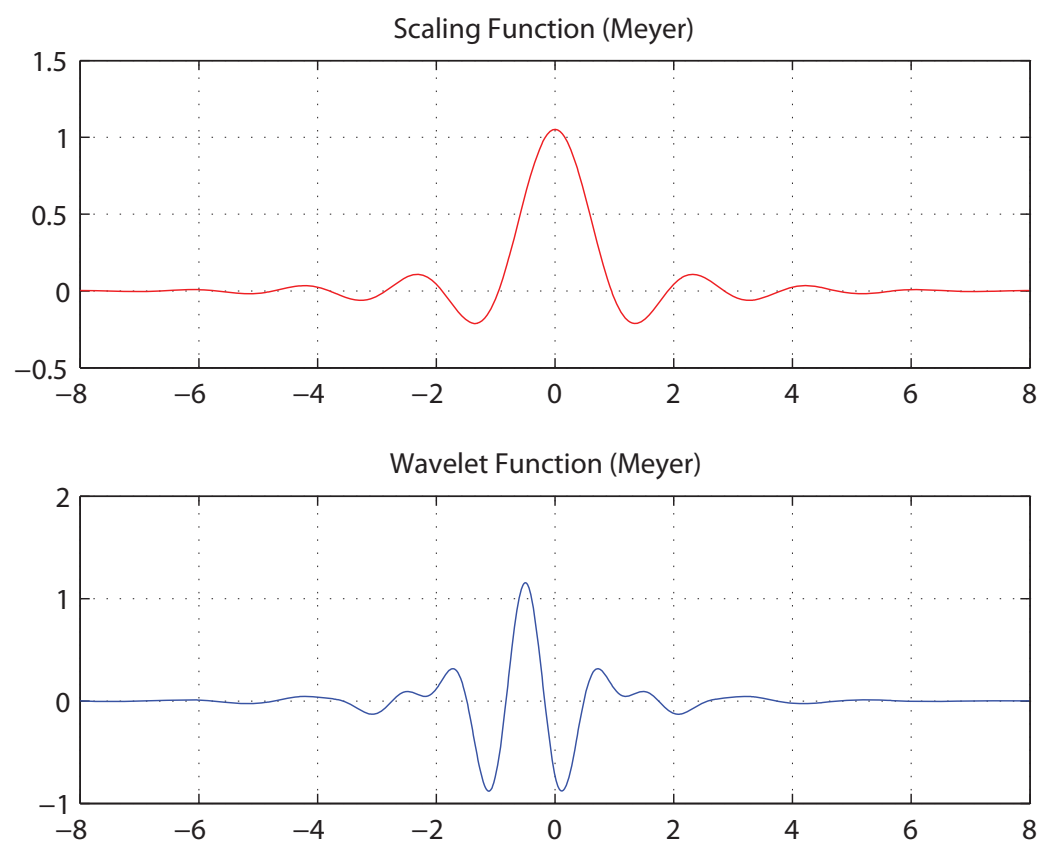

Figure 2.10: Meyer Scaling and Wavelet Function

Frere et al. [36] considered the influence of the scaling function, polynomial and exponential functions on the WT. The mean frequency was calculated for each WT and the value was compared with the mean frequency for FFT. Also, the total intensity for both WT was compared with the root mean square. Frere et al. demonstrated that the choice of the scale function had a significant difference in time and frequency domains. The authors confirmed that a second-degree polynomial scale function, as was used in von Tscharner [31] research, was suitable to be used as a reference in wavelet processing of the EMG signal. 


\subsubsection{Choosing a Wavelet for EMG Signals}

The ability of DWT to extract features from the signal is dependent on choosing an appropriate mother wavelet function. The common standard families of wavelet basis functions are Haar, Daubechies, Coiflet, Symmlet, Morlet, and Mexican Hat. Although there is not a specific rule for selecting a wavelet basis function, some features of wavelets cause a specific mother wavelet to be more appropriate for a particular application and signal type. According to Santoso et al. [37] state, for slow transients disturbances db8 and db10 wavelets were the best choice, whereas for short and fast transient disturbances db4 and db6 were more proper. Also, Walker [38] presented general guidelines for selecting a wavelet such that db4 was more appropriate for feature extraction and coiflet6 provided better data compression results. In order to select a more accurate wavelet function, it is significant that the characteristic of signal should be matched with the properties of the wavelet function.

For applying WT into the EMG signal various mother wavelets and scale functions can be used although no agreement has been reaches regarding the selection of the mother wavelet and the scale function. For example, Karlsson et al. [32] used Morlet mother wavelet with linear scale function [23,32,39] and Neto et al. [40] applied Morlet with exponential function. Von Tscharnar [31], used Cauchy mother wavelets with polynomial function. Hostens et al. [41] and Beck et al. [42] applied Daubechies mother wavelets in the EMG signal with different scale functions. Hostens et al., used linear scale function while Beck et al. used dyadic function.

The WT of the EMG signal has been used in several studies related to muscle fatigue, EMG signal processing, and muscle strength. Moreover, Neto et al. [40,43], 
and von Tscharner et al. [31,44-47], used the WT for the EMG signals that were recorded during the sports.

Flanders [48] used discrete wavelet transformation to identify time of occurrence of EMG bursts. He chose wavelet db2 and focused on the coefficient at the scale D3 because by plotting the peak to peak range of the db2 weighting coefficient values of several muscle, D3 component had the largest peak weighting coefficients based on his research. Thus, Flanders demonstrated that the simplest wavelet such as db2 was suitable for recognizing multiunit EMG bursts. Moreover, Kilby and Gholam Hosseini [49] also used mother wavelet that had different set of families to extract detailed features of the sEMG signals. They used mother wavelets that were available in LabVIEW ${ }^{\circledR}$ to decompose the sEMG signals and reconstruct the signal back. The reconstructed signals were subtracted from the original signals in order to calculate the errors. Based on different errors of the various families of the mother wavelets, they concluded the Daubechies (db5) was the most appropriate mother wavelet for analyzing sEMG signals. However, they did not consider several mother wavelets such as Morlet, Meyer, and Mexican Hat because these mother wavelets were not available by the software. Ahmed et al. [10] illustrated a comparative study of decomposing sEMG signals by using different types of wavelets. Their goal was to choose a wavelet based on the best possible energy localization in the time-scale plane. In their algorithm, they decomposed a signal using DWT for various wavelets and the energy localization in time-scale plane was calculated. From their results, db4, db5, and db6 indicated to have the highest energy compare to other wavelets for normal and healthy muscle EMG signal. 


\subsubsection{Different Studies using the Wavelet Transform}

\section{MUAP}

Wavelet analysis is able to provide a new way in describing MUAP in the timefrequency plane. Guglielminotti and Merletti [50] theorized that in wavelet analysis, it was required to choose a wavelet which was the best match for the shape of the MUAP. The results of WT provided the best possible energy localization in the time-scale plane. Pattichis and Pattichis [51] utilized the WT to analyze signals at different resolution levels and this process was known as multi-resolution analysis. They considered the connection between wavelet coefficients and the time-frequency plane and found out how coefficients from one stage of the WT could be used to construct functional approximation to the original signal. In addition, Laterza and Olmo [52] proposed WT as an effective tool for the MUAP detection. They used the Mexican Hat wavelet because it matched with the shape of the MUAP, and the result of WT provided the best possible energy localization in time-scale plane. Also, Zennaro et al. [53] provided a decomposition method based on multichannel longterm recording of signal instead of single-channel recording. They believed that the wavelet coefficients of lower frequency bands were more important than higher bands for distinction of action potential characterization. They developed a software and believed that it is appropriate for the study of MU patterns and recruitment order in long-term recording of signal. However, the results from Yamada et al.'s [54] experiment indicated that the high frequency information was also significant in classifying MUAP. They applied principal component analysis (PCA) to wavelet coefficients in order to consider all frequency informations. 


\section{Fatigue}

The EMG signal can be used to detect muscle fatigue. According to Luca [55], the fatigue was related to the various physiological and psychological phenomena. Fatigue is defined as a condition when the muscle's ability to contract and produce force is decreased. The frequency domain provides significant features of the EMG signal [56]. Therefore, in the frequency domain several variables of EMG power spectrum requires to be analyzed.

- Mean frequency: The mean frequency (MNF) is an average frequency which is defined as a sum of product of the power spectrum and the frequency over a total sum of the power spectrum $[57,58]$. Eq 2.5 shows the definition of MNF:

$$
M N F=\frac{\sum_{j=1}^{M} f_{j} P_{j}}{\sum_{j=1}^{M} P_{j}}
$$

- Median frequency: The median frequency (MDF) is a frequency value at which the EMG power spectrum is divided into two regions with an equal power. Eq 2.6 shows the corresponding formula:

$$
\sum_{j=1}^{M D F} P_{j}=\sum_{j=M D F}^{M} P_{j}=\frac{1}{2} \sum_{j=1}^{M} P_{j}
$$

The MNF and MDF are used for stationary signals. When a muscle contracts during dynamic conditions, the EMG signal generated by the muscle should be considered as a non-stationary signal. Meanwhile, the analysis of the non-stationary signal requires the time-frequency spectrum. The instantaneous mean and median 
frequency are derived by replacing the power spectrum with the time-frequency spectrum. Thus, the IMNF and IMDF are computed through following equations [59]:

$$
\begin{gathered}
\int_{0}^{I M D F(t)} S(t, f) d f=\int_{I M D F(t)}^{\infty} S(t, f) d f \\
I M N F(t)=\frac{\int_{0}^{\infty} f S(t, f) d f}{\int_{0}^{\infty} S(t, f) d f}
\end{gathered}
$$

In general, the mean frequency can be extracted by applying a FFT or a WT. Various papers have been measuring or comparing the frequency from FFT and WT $[31,46,60-66]$ to find the best method for analyzing the sEMG signals.

Constable et al. [67] analyzed sEMG signals from four muscles (soleus, vasti, gluteus maximus, and hamstrings) during dynamic contraction using continuous DWT based on 4-coefficient Daubechies wavelet. The results illustrated more high frequency activation at low levels and more low frequency activation at higher levels due to fatigue of fast twitch fibers.

Karlsson et al. [32] stated that the mean frequency was usually measured from power spectrum which was attained by a Fourier Transform. Moreover, the mean frequency could also be gained from a wavelet based power spectrum. Barandum et al. [33] affirmed the difference between using Fourier Mean Frequency (FMF) and a Wavelet Mean Frequency (WMF) when analyzing sEMG during early fatigue in isometric contraction. They demonstrated that the wavelet transform was able to measure the mean frequency in a shorter time period. In addition, Beck et al. [42] aimed to determine the mechanomyographic (MMG) and EMG center frequency (mean power frequency $(\mathrm{MPF})$, median frequency $(\mathrm{MDF})$, and wavelet center frequency $(\mathrm{CF}))$ pat- 
terns for biceps brachii muscle during fatiguing isokinetic actions by comparing FFT and DWT methods. For DWT, Beck used the Daubechies 4 (db4) wavelet algorithm. The DWT decomposed MMG and EMG signal into several scales (frequency bands) and the center frequency was defined after plotting the amplitude-frequency distribution. As a result, they found out that the mpf, mdf, and cf were the same for MMG and EMG. Also, the Fourier methods were suitable for providing the patterns for normalized MMG and EMG center frequency during fatiguing dynamic muscle actions. Karlsson et al. [23,32] reported that the WT was more accurate for determining the EMG power spectrum and produced mean frequency evaluated which has less noise compare to the estimates from the FT. Moreover, Kumar et al. [65] used WT for diagnosing fatigue. They proposed that the WT decomposed the signal into several multi-resolution components based on wavelet function when the sampling rate is 5 $\mathrm{kHz}$. They used WT with various WF to decompose the sEMG signal and the output power was calculated. According to the output power parameter, Kumar et al. chose the WF that provided the best contrast between non-fatigue and fatigue sEMG signals. They believed that the difference between non-fatigue and fatigue muscles was emphasized when sym4 and sym5 wavelet functions were used. The muscle fatigue using sym4 and sym5 at level 8 and 9 (out of 10) of wavelet decomposition could be determined by applying WT to sEMG signals.

\section{Classification of EMG signal}

Gheab et al. [68] presented a method to analyze different EMG signals by studying the coefficients of fifteen wavelet families after passing the training and validated in the neural network (NN). They considered the EMG signal that was recorded from right pectoralis, right biceps, and right triceps muscles for three volunteers when 
performing the butterfly exercises with $5 \mathrm{lb}$ and $10 \mathrm{lb}$. Their work comprised of using WT technique and applying Artificial Neural Network (ANN). WT was used to generate denoised and compressed EMG signal and ANN was applied for feature extraction and classification. They concluded that Haar and db in level 4 and 5 were more comfortable to be used with the recorded EMG signal. In addition, Ozsert et al. [69] proposed methods to classify biceps, frontallis, and abductor pollicis brevis muscles after recording EMG signals. They reduced the size of EMG signals by using the WT (pre-process EMG signals) and an auto-regressive (AR) method. The three ANN types were used for classifying EMG signals such as multiplayer perceptron (MLP), radial base function (RBF), and general regression neural networks (GRNN). The AR coefficients were used for training and testing MLP, RBF, and GRNN. Ozsert et al. showed that the classification accuracy of the pre-processed data set was higher compared to unprocessed data set for MLP and GRNN systems.

Von Tscharner analyzed EMG signals based on the wavelet transform [31] and produced intensity patterns of the EMG signal. Von Tscharner and Goepfert [45] combined the intensity patterns into a multi-muscle pattern (MMP) and represented it in a pattern space that is a vector space. Then, Von Tscharner used the spherical classification to classify the EMG intensity patterns [70]. As a result, an optimal dimensionality for the pattern space was required at which the spherical classification worked best. If the dimensionality was fixed too low, a pattern space was unable to reproduce the patterns with details. Furthermore, limiting the dimensionality decreased the noise in the reproduced patterns and improved classification. 


\section{Chapter 3}

\section{Methodology}

This chapter presents the proposed methodology for analyzing EMG signals to identify healthy controls and FAI patients. It also refers to the procedures done to the signals for pre-processing, processing and analyzing the EMG signals.

Figure 3.1 provides a schematic description of the steps discussed in this research. In the first step, the raw EMG was processed by generating the EMG linear envelope which was provided based on normalization, full-rectified, and low-pass filtering. This process gave useful results for analysis. Then the integrated EMG was calculated for comparison. In the second step, the wavelet transform method was applied to the normalized EMG signals. Different wavelet functions were applied and their wavelet coefficients were calculated. One of the wavelet functions that provided the highest energy was selected. The third step of this producer was to calculate the wavelet power spectrum from the wavelet coefficients of the selected wavelet function. 


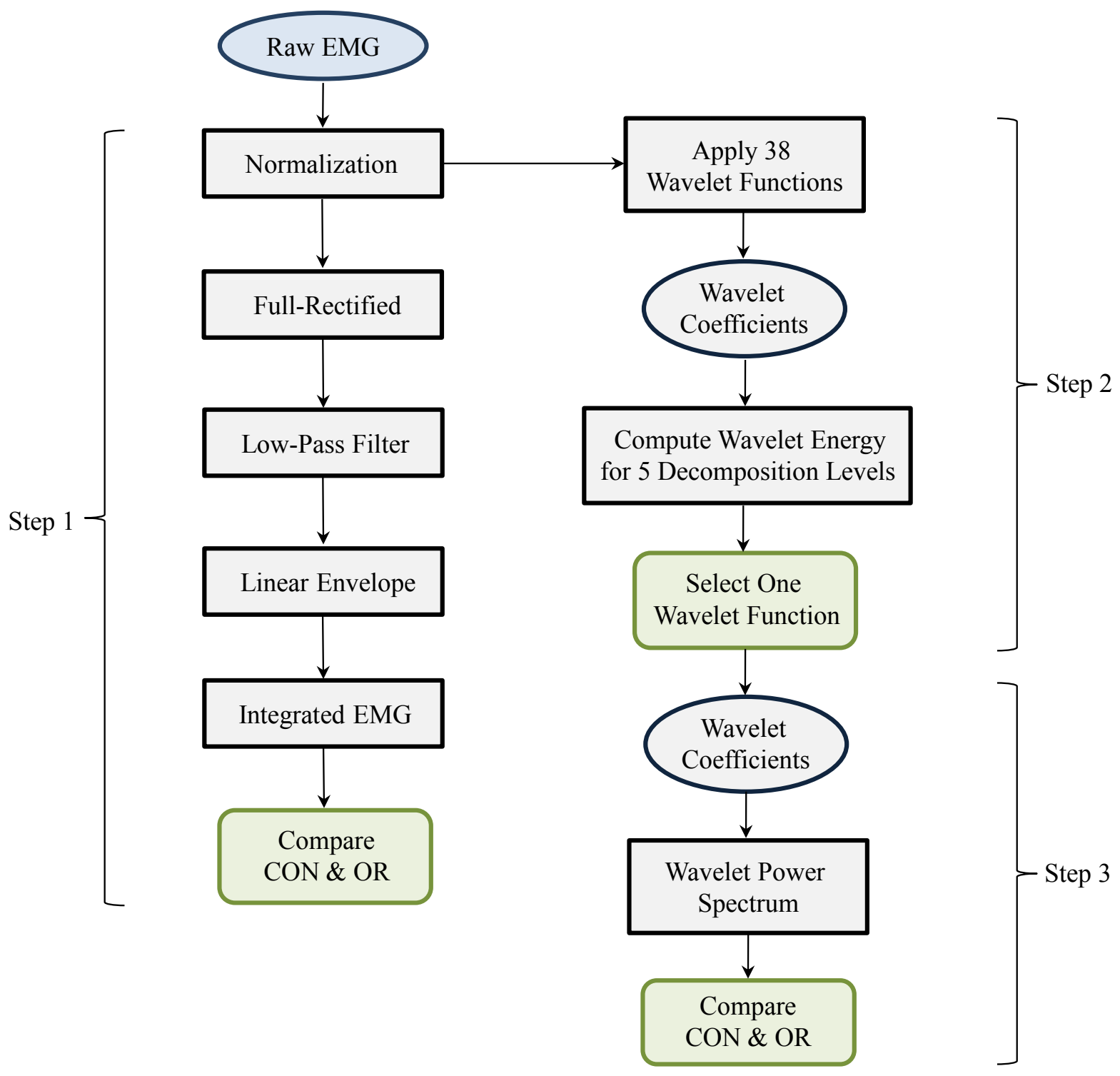

Figure 3.1: Schematic Description of the Technique

\subsection{Recording Process for EMG Signals}

The EMG data of Gluteus Maximus (GMax), Tensor Fasciae Latae (TFL), and Rectus Femoris (RF) muscles were recorded from 30 subjects during dynamic contraction 
of squat task (Figure 3.2). During squat, subjects were required to stand with feet shoulder-width apart, parallel to one another. Both arms were anteriorly extended, and heels were in contact with the floor during the entire squat. Five repetitions of the same movement were executed [6]. The recorded sEMG signals formed two groups: control and FAI. The control group consisted of 15 males and 1 female whereas FAI group contained 11 males and 3 females. The file which was a structure array contained the data for all the subjects who belonged to control (CON) and FAI presurgery patients (OR). The FAI group was named OR in this file as the patients were waiting for surgery.
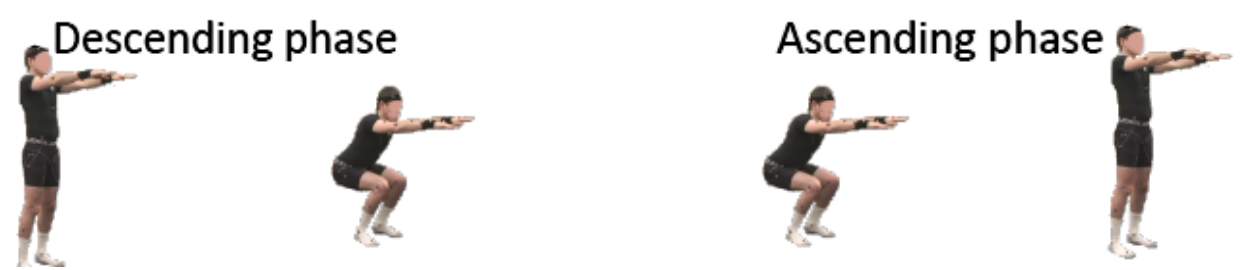

Figure 3.2: Descending, Stationary, and Ascending Phase of Squat [6]

Participant characteristics are indicated in Table 3.1. 
Table 3.1: Participant Characteristics by Group

\begin{tabular}{|c|c|c|c|}
\hline Group & Gender & Weight $(\mathrm{Kg})$ & Height $(\mathrm{cm})$ \\
\hline \multicolumn{4}{|c|}{ Control (CON) Subjects } \\
\hline 1 & M & 104.591 & 191.2 \\
\hline 2 & M & 132.009 & 179 \\
\hline 5 & M & 113.527 & 176 \\
\hline 6 & $\mathrm{~F}$ & 97.245 & 152 \\
\hline 7 & M & 120.189 & 180.5 \\
\hline 10 & M & 93.078 & 175.5 \\
\hline 11 & M & 83.666 & 180.5 \\
\hline 12 & M & 87.466 & 168 \\
\hline 15 & M & 117.896 & 177.5 \\
\hline 17 & M & 83.551 & 178 \\
\hline 19 & M & 83.311 & 181 \\
\hline 20 & M & 106.529 & 183 \\
\hline 22 & M & 82.646 & 177 \\
\hline 23 & M & 147.725 & 176 \\
\hline 24 & M & 61.129 & 160.5 \\
\hline 25 & $\mathrm{M}$ & 79.526 & 175.5 \\
\hline \multicolumn{4}{|c|}{ FAI (OR) Subjects } \\
\hline 3 & M & 129.012 & 183.75 \\
\hline 4 & M & 83.035 & 168 \\
\hline 8 & M & 139.491 & 186 \\
\hline 9 & M & 133.394 & 176.5 \\
\hline 13 & M & 126.997 & 174.2 \\
\hline 14 & M & 101.154 & 175 \\
\hline 16 & M & 108.719 & 175 \\
\hline 18 & M & 149.428 & 176 \\
\hline 21 & $\mathrm{~F}$ & 97.508 & 163 \\
\hline 26 & M & 64.312 & 167 \\
\hline 27 & M & 136.712 & 167 \\
\hline 28 & $\mathrm{M}$ & 120.632 & 175 \\
\hline 29 & $\mathrm{~F}$ & 132.507 & 168.5 \\
\hline 30 & $\mathrm{~F}$ & 59.766 & 167 \\
\hline
\end{tabular}




\subsection{EMG Data Processing}

Raw EMG signals were recorded from GMax, TFL, and RF muscles had a zero hertz (DC) offset, therefore the DC offset was removed from each raw EMG signal by using detrend function which removes linear trend from the matrix. In order to be able to compare the EMG activity in the same muscle on different subjects, the EMG signals have to be normalized. Normalization of EMG signals were performed by dividing EMG signals during a squat task to a reference EMG value achieved from the same muscle of the same subject. There are various normalization methods available to do so and choosing between them is critical as it can influence the amplitude and pattern of EMG signals [71]. However, there is not a completely optimized method for normalization of EMG signals therefore, a variety of methods have been used to gain the normalization reference values. In this research we utilized MVIC method for normalization. In order to measure the MVIC the participants were asked to lie down on a testing bench, which was provided with support beams and adjustable straps to limit the movement of the limbs and hold it in place during isometric contraction. The MVIC were collected for duration of 5 seconds for each muscle. MVICs for GMax and RF muscles were measured when the participants leg was straight, and they were asked to push upwards against the Hand-Held Dynamometer (HHD). Moreover, MVIC for TFL muscle was collected when the participants were asked to push diagonally against the HHD. The MVIC values of participants for three muscles are indicated in Table 3.2. 
Table 3.2: Participants' MVIC Values for GMax, TFL, and RF Muscles

\begin{tabular}{|c|c|c|c|}
\hline \multicolumn{4}{|c|}{ MVC } \\
\hline Subjects & GMax (muscle 1) & TFL (muscle 2) & RF (muscle 3) \\
\hline 1 & $7.91 E^{-2}$ & $4.26 E^{-1}$ & $2.37 E^{-1}$ \\
\hline 2 & $6.20 E^{-2}$ & $3.74 E^{-2}$ & $1.16 E^{-1}$ \\
\hline 3 & $7.28 E^{-2}$ & $3.78 E^{-1}$ & $1.18 E^{-1}$ \\
\hline 4 & $3.95 E^{-4}$ & 0 & $2.52 E^{-4}$ \\
\hline 5 & $5.51 E^{-2}$ & $7.02 E^{-2}$ & $1.14 E^{-1}$ \\
\hline 6 & $5.40 E^{-2}$ & $1.74 E^{-1}$ & $9.17 E^{-2}$ \\
\hline 7 & $5.10 E^{-2}$ & $1.03 E^{-1}$ & $5.31 E^{-2}$ \\
\hline 8 & $1.31 E^{-4}$ & $1.35 E^{-4}$ & $3.03 E^{-4}$ \\
\hline 9 & $2.88 E^{-4}$ & $1.04 E^{-4}$ & $1.94 E^{-4}$ \\
\hline 10 & $7.34 E^{-5}$ & $3.55 E^{-4}$ & $1.96 E^{-4}$ \\
\hline 11 & $1.18 E^{-4}$ & $3.70 E^{-4}$ & $3.93 E^{-4}$ \\
\hline 12 & $1.23 E^{-4}$ & $9.30 E^{-4}$ & $2.06 E^{-4}$ \\
\hline 13 & $4.62 E^{-5}$ & $2.94 E^{-4}$ & $1.53 E^{-4}$ \\
\hline 14 & $1.74 E^{-4}$ & $8.11 E^{-4}$ & $2.77 E^{-4}$ \\
\hline 15 & $2.01 E^{-4}$ & $7.54 E^{-4}$ & $3.09 E^{-4}$ \\
\hline 16 & $1.69 E^{-4}$ & $2.98 E^{-4}$ & $2.44 E^{-4}$ \\
\hline 17 & $1.90 E^{-4}$ & $5.25 E^{-4}$ & $2.55 E^{-4}$ \\
\hline 18 & $9.20 E^{-5}$ & $2.40 E^{-4}$ & $1.16 E^{-4}$ \\
\hline 19 & $2.74 E^{-2}$ & $1.10 E^{-1}$ & $9.24 E^{-2}$ \\
\hline 20 & $7.26 E^{-5}$ & $1.35 E^{-4}$ & $1.16 E^{-4}$ \\
\hline 21 & $7.97 E^{-5}$ & $2.79 E^{-4}$ & $1.99 E^{-4}$ \\
\hline 22 & $1.93 E^{-4}$ & $2.35 E^{-4}$ & $1.87 E^{-4}$ \\
\hline 23 & $1.30 E^{-4}$ & $4.25 E^{-4}$ & $2.43 E^{-4}$ \\
\hline 24 & $1.05 E^{-4}$ & $8.90 E^{-5}$ & $1.93 E^{-4}$ \\
\hline 25 & $1.29 E^{-1}$ & $2.44 E^{-1}$ & $9.82 E^{-2}$ \\
\hline 26 & $1.65 E^{-4}$ & $5.02 E^{-4}$ & $1.97 E^{-4}$ \\
\hline 27 & $5.78 E^{-2}$ & $3.11 E^{-1}$ & $2.00 E^{-1}$ \\
\hline 28 & $7.17 E^{-2}$ & $2.93 E^{-1}$ & $2.41 E^{-1}$ \\
\hline 29 & $7.57 E^{-5}$ & $1.12 E^{-4}$ & $1.55 E^{-4}$ \\
\hline 30 & $1.60 E^{-2}$ & $8.09 E^{-2}$ & $8.50 E^{-2}$ \\
\hline
\end{tabular}

After normalization, EMG signals were full-rectified which means were taking the absolute value of the signal. The rectified signals were passed through a butterworth $5^{\text {th }}$ order low pass filter with cut off frequency of $10 \mathrm{~Hz}$. The sampling frequency of the 
signal is $1000 \mathrm{~Hz}$. This process provided the linear envelope of signals. Furthermore, the filtered signals were amplitude-normalized to the peak MVIC EMG (nEMG) and then integrated in order to produce the integrated EMG (IEMG) values. The IEMG define as

$$
I E M G=\int_{t_{i}}^{t_{f}} n E M G(t) d t
$$

where $n$ is the number of points in EMG grap. Also, $t_{i}$ and $t_{f}$ are initial and final time of recording respectively.

After normalization, the outliers were eliminated from the data set. This was estimated by plotting the normalization graph for each subject. If the subjects normalization graph were above $100 \%$ (the normalized graph should be between 0 to 1 or $0-100 \%$ ), the subject was eliminated from the data set. Figure 3.3, 3.4, 3.5 show the normalized graphs. The blue and red colors are related to $\mathrm{CON}$ and OR group respectively. By referring to Figure 3.3, there were two outliers in muscle 1 (subject 28, and 30) of OR group. In Figure 3.4 which is related to EMG signals for muscle 2, all EMG normalized graphs were in the range. However, in Figure 3.5, we had an outlier (subject 20) for CON group. Moreover, there were some subjects who had problems during the acquisitions. In other words, the EMG system had problems. Therefore, some channels were null (series of zeroes) and these subjects were removed from the data set (subject 4 and 26). 


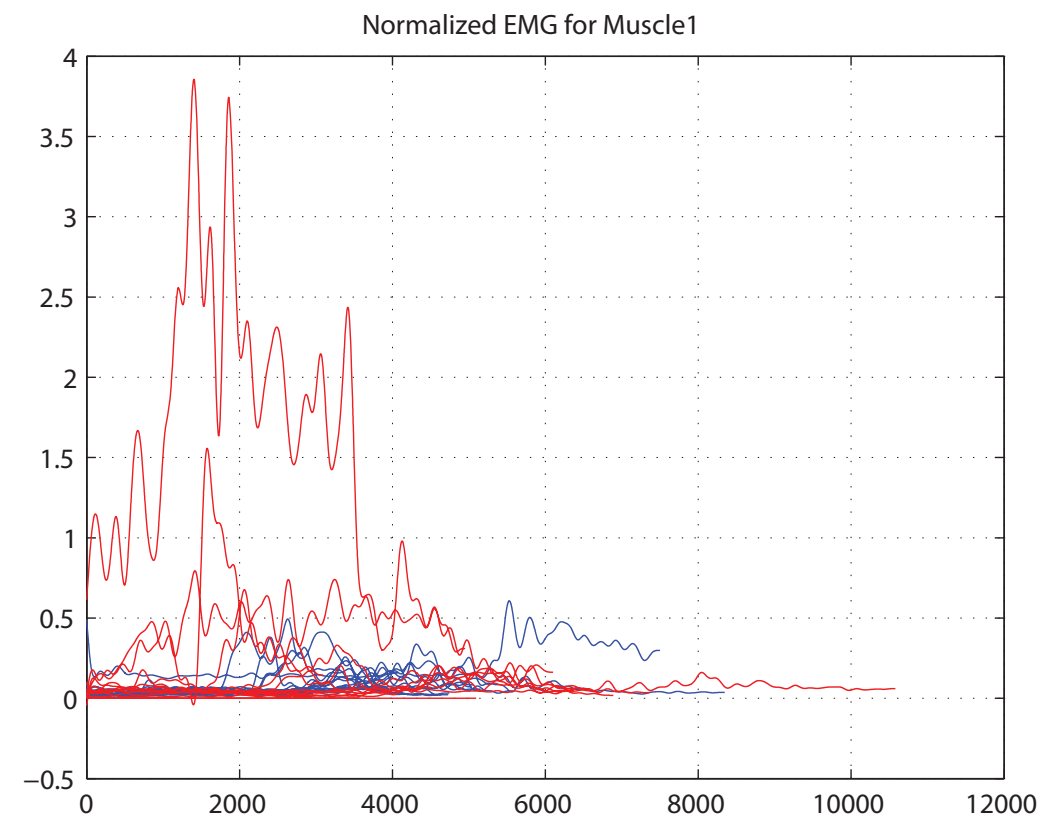

Figure 3.3: Normalized EMG Signals for CON (blue) and OR (red), Muscle 1

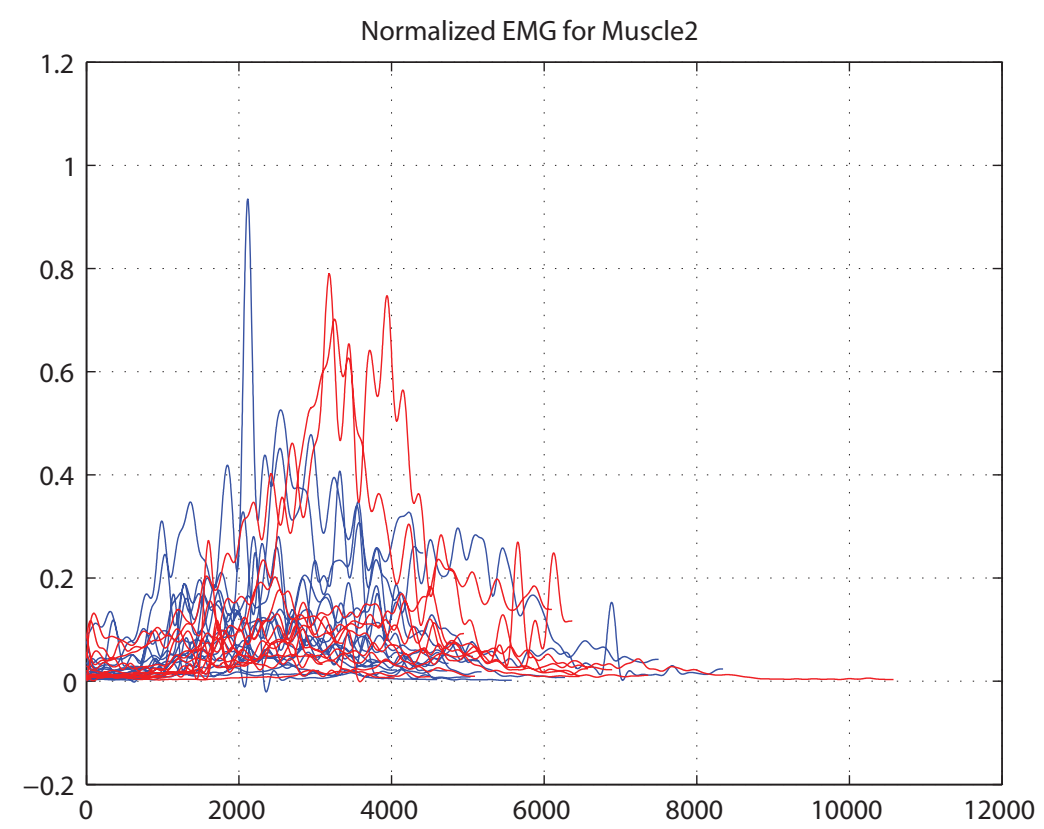

Figure 3.4: Normalized EMG Signals for CON (blue) and OR (red), Muscle 2 


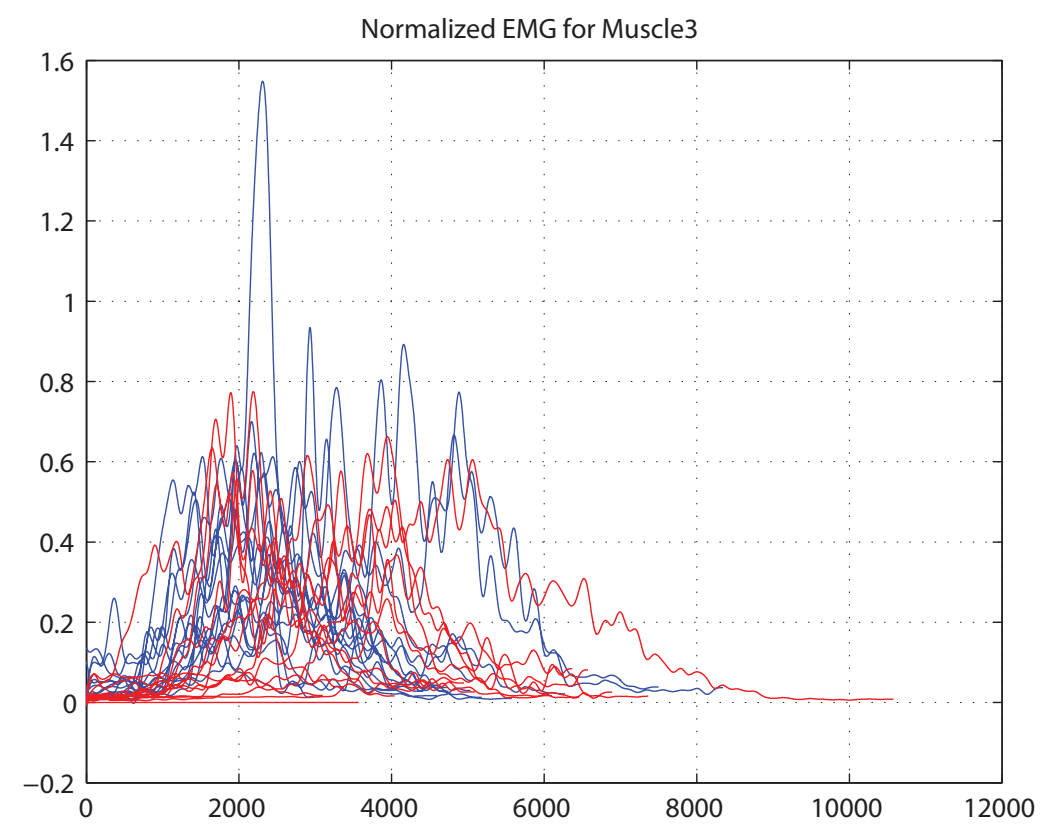

Figure 3.5: Normalized EMG Signals for CON (blue) and OR (red), Muscle 3

\subsection{EMG Analysis Using Wavelet Transforms}

Data analysis was performed using MATLAB programming language with the signal processing and Wavelet toolboxes (The Math Works, INC.). The EMG values were recorded from GMax, TFL, and RF muscles of the affected sides of the 30 subjects. There were 5 repetitions for every subject with sampling frequency of $1000 \mathrm{~Hz}$. The size of the samples in EMG data set was large, thus EMG signals were pre-processed by using the wavelet transform. As the size of EMG signals were reduced, unnecessary details were eliminated. EMG signals were recorded during squat cycles, thus each recorded signal was divided into three regions based on squat phases.

The analysis of the data file was started with converting it to .mat file and then removing any DC offset in order to be ready for the wavelet families' analysis. The 
wavelet analysis was performed by a function called "analytical wavelet" or "mother wavelet". There are different families or set of mother wavelets in Wavelet toolbox which differ in their mathematical principles named as Haar, Daubechies (db1 to db10), Symlets (sym2 to 8), Coiflets (coif1 to 5), Biorthogonal (bior1.1 to 6.8), Reverse biorthogonal (rbio1.1 to 6.8), Meyer (meyr), Discrete approximation of Meyer (dmey), Gaussian (gaus1 to 8), Mexican hat (mexh), and Morlet (morl). EMG signals were decomposed using DWT with various WFs. From the wavelet toolbox menu, we used discrete wavelet 1-D option which allowed us to decompose our EMG signals based on Haar, Daubechies (db1 to db10), Symlets (sym2 to 8), Coiflets (coif1 to 5), Biorthogonal (bior1.1 to 6.8), and discrete approximation of Meyer (dmey) mother wavelets. Moreover, MATLAB code was written for applying DWT to EMG signals. The WT decomposes a signal into several multi-resolution (levels) components based on basis functions or WFs. These WFs are achieved by dilation, contraction, and shifts of a unique function. Decomposition of the signal into basis of wavelet functions implies the computation of the inner products between the signal and the basis function, leading to a set of coefficients called wavelet coefficients.

\subsubsection{Level of Decomposition}

From Chapter 2 of this thesis, we have known that the wavelet transform is organized by different levels. The maximum level to apply the wavelet transform depends on how many data points contained in our data set, while there is a down-sampling by 2 operation from one level to the next level. In our analysis, we used 5 levels of decomposition. 


\subsubsection{Multi-Resolution Analysis}

To compute multilevel wavelet decomposition, 38 different wavelet functions exerted at decomposition level 1 to 5 . The wavelet coefficients from each wavelet function were used to calculate the energy of the sEMG signals for each subject in each phase.

\subsubsection{Wavelet Selection Based on Energy Calculation}

The wavelet energy was computed for approximation and detail coefficients. $E_{a}$ is the percentage of energy corresponding to the approximation and $E_{d}$ is the vector containing the percentage of energy corresponding to the details. $E_{d}$ for each sEMG signal was collected after using 38 wavelet functions and the highest five energies were highlighted for each subject in three phases. Then, the highest repetition wavelet function was chosen as our wavelet function. This procedure is indicated in Figure 3.6.

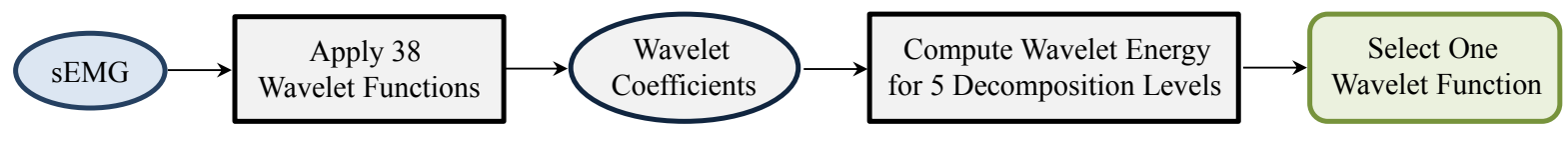

Figure 3.6: Procedure of Selecting a WF 


\subsubsection{Wavelet Power Spectrum}

Wavelet power spectrum is a way to determine the distribution of energy along the sEMG signal. In this procedure wavelet coefficients of the selected wavelet function was computed and the power spectrum was measured during 5 levels of decomposition. The distribution of power for each muscle during descending, stationary, and ascending phase for both $\mathrm{CON}$ and $\mathrm{OR}$ groups was determined and plotted. Figure 3.7 shows the block diagram for computing power and discriminating two groups based on their power spectrum.

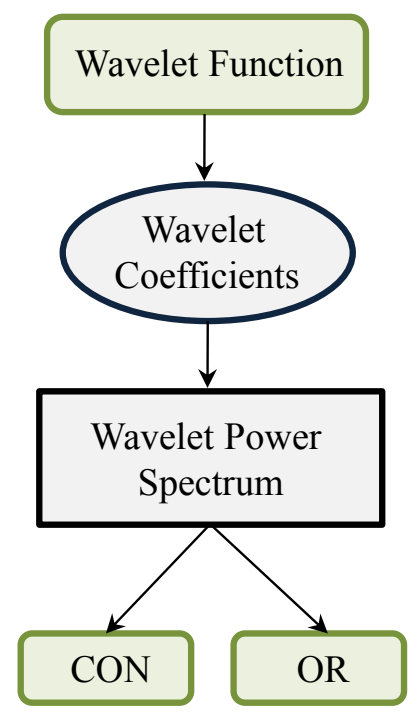

Figure 3.7: Block Diagram for Computing Power Spectrum 


\section{Chapter 4}

\section{Analysis of Results}

In this chapter, we will first describe how the EMG signals were analyzed. We introduce analysis methods and data used to achieve the methodology presented in chapter 3. Later, we explain the various procedures and present the results and analysis.

The algorithm developed in this study evaluated for its performance by discriminating the $\mathrm{CON}$ and $\mathrm{OR}$ group from one another. The comparisons were made by three different procedures including:

1. Computing IEMG values

2. Selecting a wavelet function

3. Computing the wavelet power spectrum 


\subsection{Data Selection}

In this study, the acquisition of the EMG signal achieved from a total of 30 subjects, which consist of $16 \mathrm{CONs}$ and $14 \mathrm{ORs}$, to perform a squat activity. The "Dataset.mat" file contained the data for all subjects. Within a subject there were various fields such as; MVIC, Trial, Group, Gender, Height, Weight.

- Maximum Voluntary Isometric Contraction (MVIC): The maximum value of tension a muscle can generate and hold was collected and referred to GMax, TFL, and RF of the affected side of the subject.

- Trial: The trial field contained 5 columns (structure array of $1 \times 5$ ) as there were 5 repetitions for every subject. Each column of this trial contained the AnChEMG (analog channel EMG) and Events fields. The AnChEMG was a matrix nx3, where $\mathrm{n}$ was the number of frames (duration of the acquisition with sampling frequency of $1000 \mathrm{~Hz}$ ) and the 3 was the number of muscles (GMax, TFL, RF which are Muscle1, Muscle2, and Muscle3 respectively). Moreover, the events field included a 4 number array corresponding to 1-start of descending phase of squat, 2-end of descending phase, 3-start of ascending phase, 4-end of ascending phase respectively. Figure 3.2 shows these events.

- Group, gender, height, and weight: In chapter 3, Table 3.1 illustrates participant characteristics.

EMG data were recorded from GMax, TFL, and RF muscles during dynamic contraction for descending, stationary, and ascending phases. After removing the DC offset from sEMG signals and normalizing them some of the sEMG graphs were out 
of range of normalization (0-1). Thus, these subjects were considered as outliers.

EMG signals during squat cycles were normalized by dividing the EMG signal of each muscle by the MVIC related to that specific muscle for each subject. Then, EMG signals were full-rectified and passed through a butterworth low pass filter $\left(5^{\text {th }}\right.$ order) with the $10 \mathrm{~Hz}$ cutoff frequency. The result of this process was the linear envelopes of signals. The filtered signals were integrated and IEMG values were computed for both CON and OR groups in order to distinguish between them. The IEMG graphs for Gmax, TFL, and RF muscles (muscle 1, 2, and 3) are plotted in Figure 4.1, 4.2, 4.3, respectively. Moreover, IEMG values for each muscle are provided in appendix A.

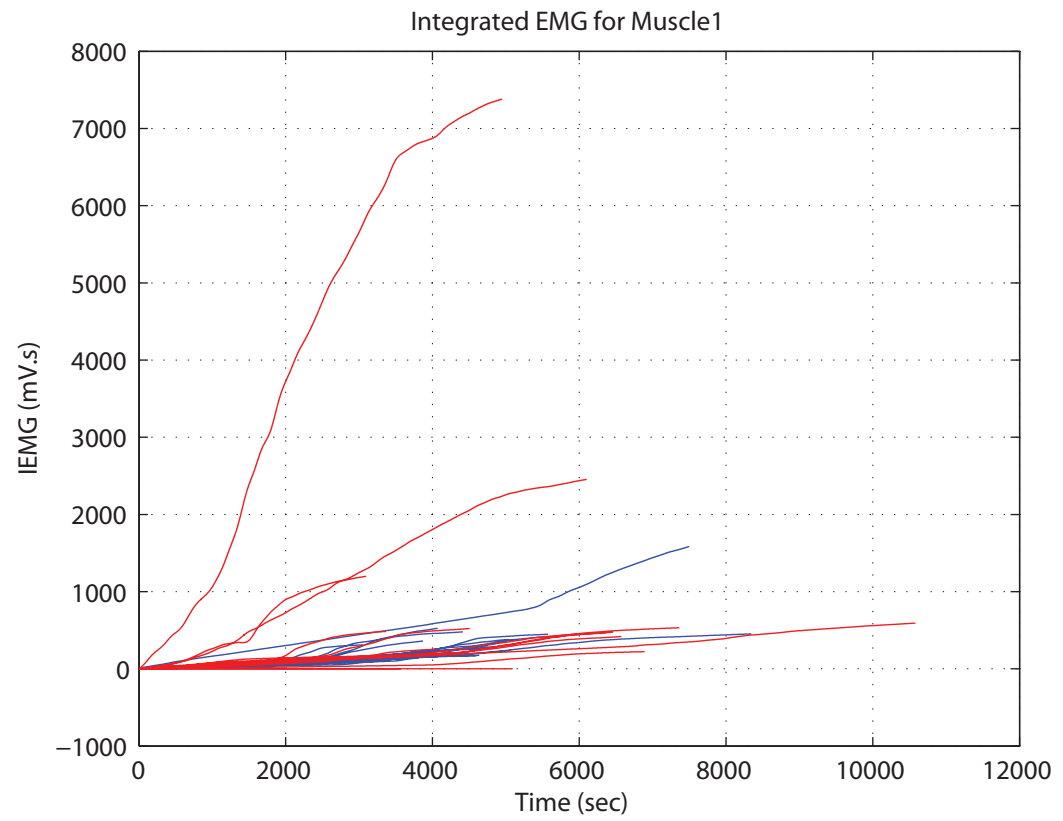

Figure 4.1: Integrated EMG Graphs for CON (blue) and OR (red), Muscle 1 


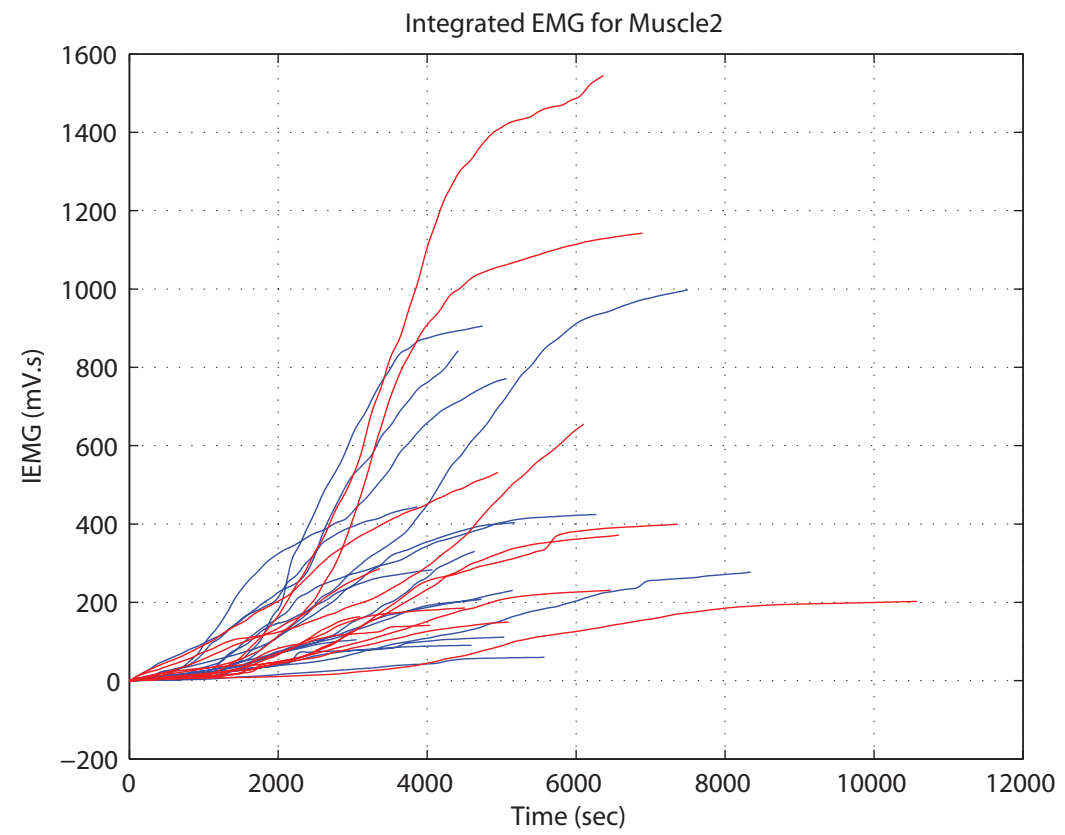

Figure 4.2: Integrated EMG Graphs for CON (blue) and OR (red), Muscle 2

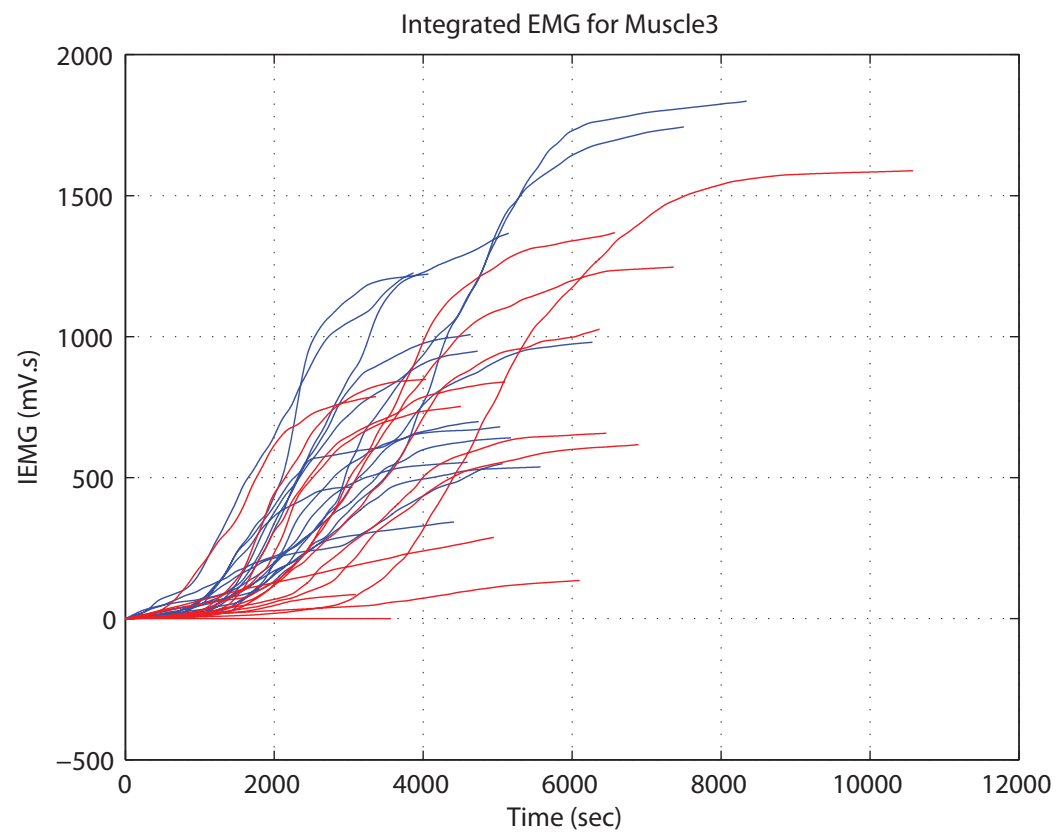

Figure 4.3: Integrated EMG Graphs for CON (blue) and OR (red), Muscle 3 
Then, IEMGs were time normalized by dividing them to the length of signals. The IEMG and time normalized values for CON and OR groups are provided in tables

Time Normalize IEMG for Muscle 1

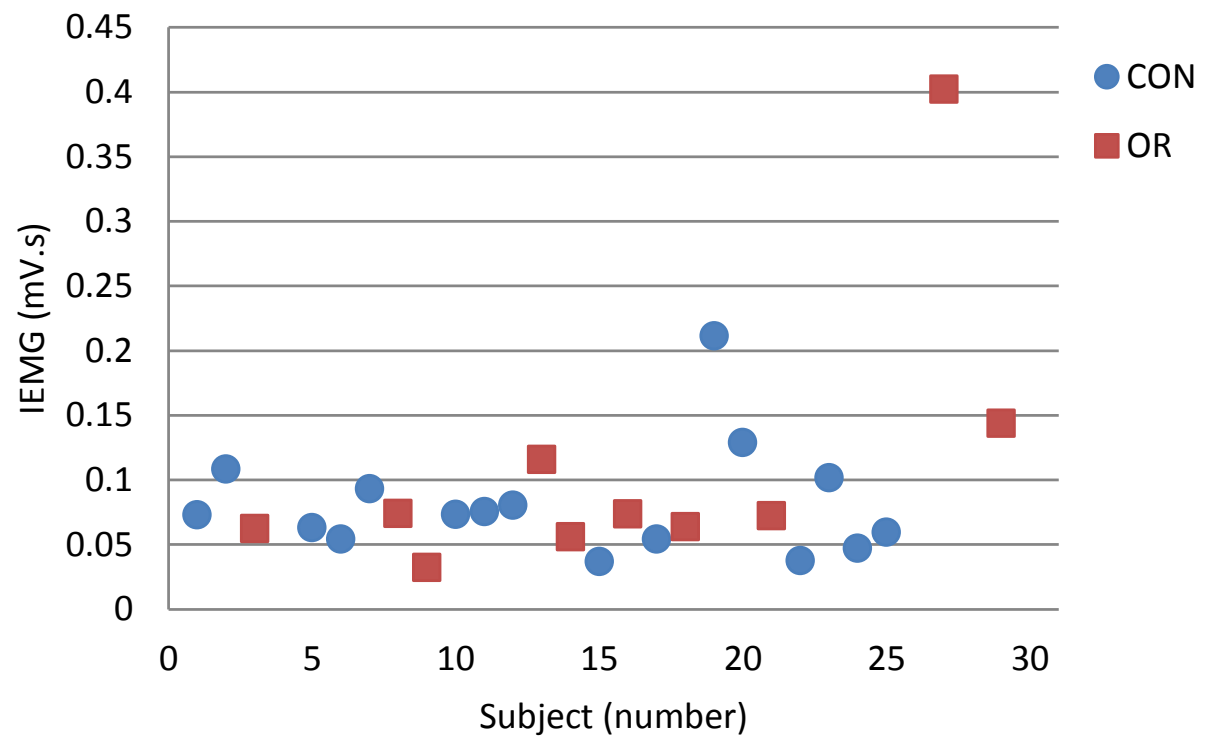

Figure 4.4: Time Normalized IEMG for Muscle 1 
Time Normalize IEMG for Muscle 2

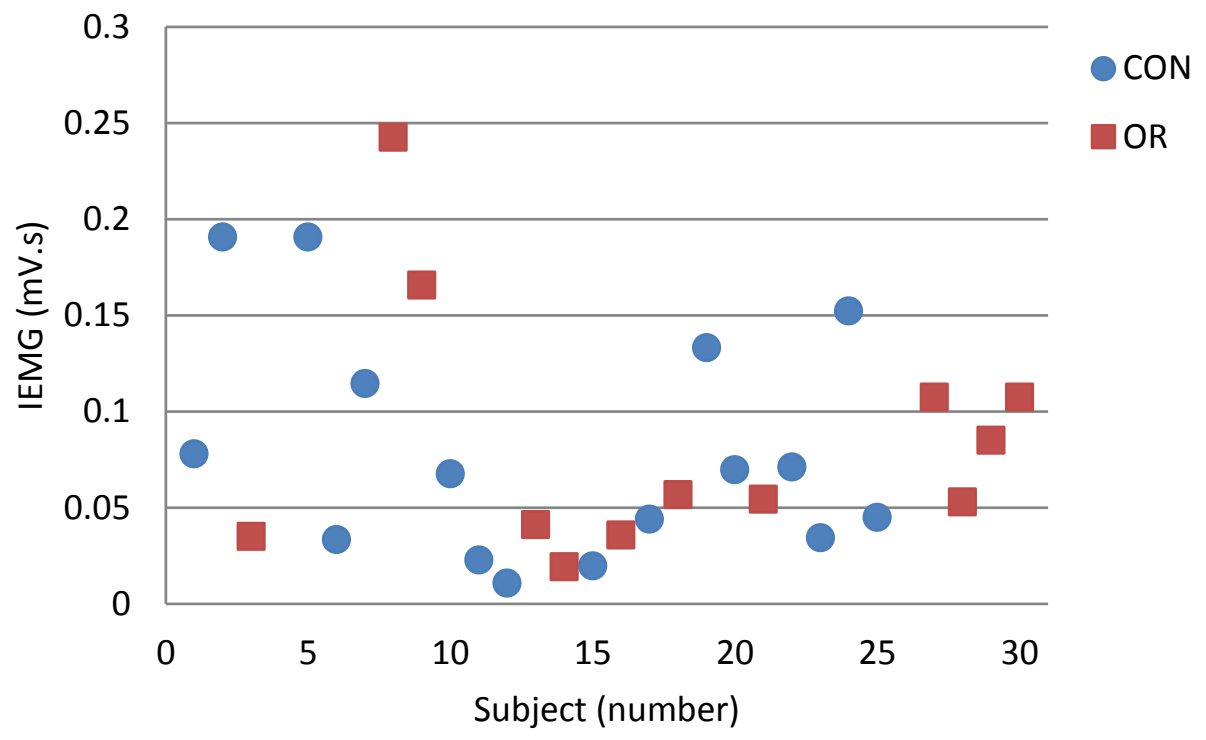

Figure 4.5: Time Normalized IEMG for Muscle 2 
Time Normalized IEMG for Muscle 3

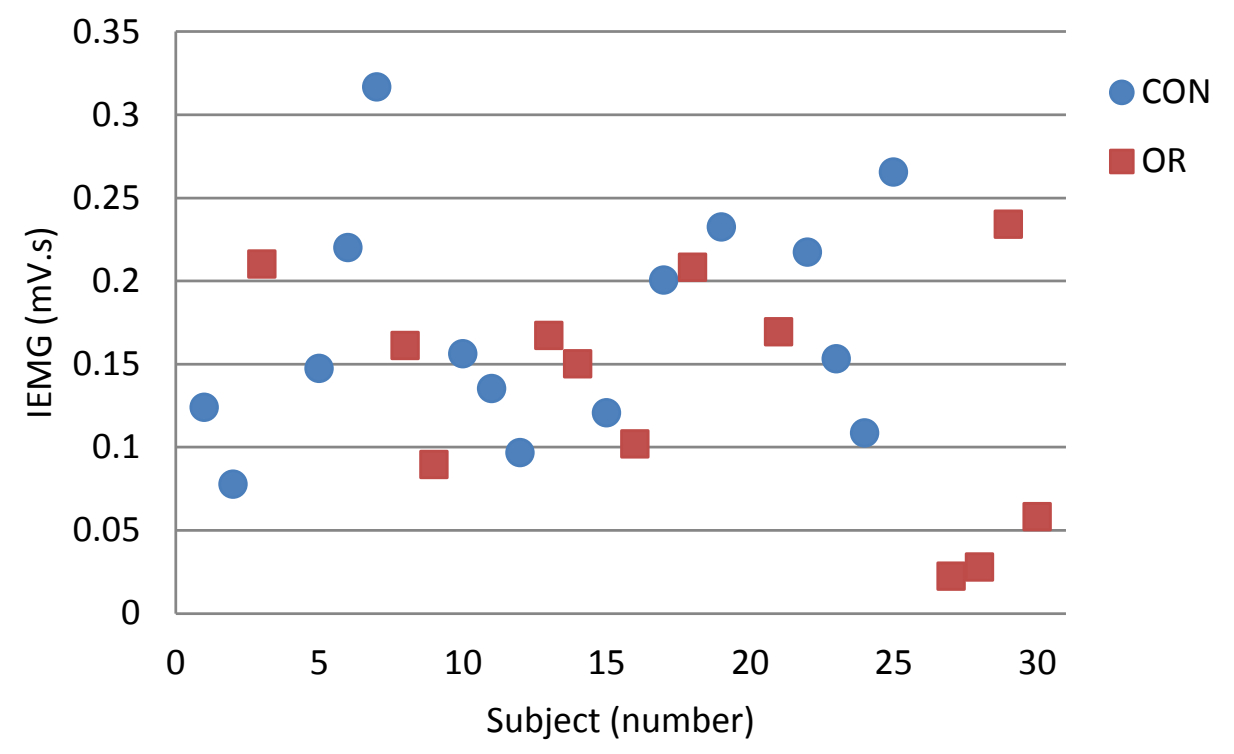

Figure 4.6: Time Normalized IEMG for Muscle 3

The results obtained show that the two groups are not separable based on their IEMG value and even time normalized IEMG graphs. Thus, time-frequency methods will be used in order to convert the signal from time domain to frequency domain.

\subsection{Wavelet Transforms}

The acquired EMG data were analyzed in MATLAB. Various wavelets were utilized to analyze raw sEMG signals. The DWT was applied to EMG signals which collected from GMax, TFL, and RF muscles (muscle 1, 2, and 3 respectively). The mother wavelets in this study include Haar, Daubechies 1 to 10, Symlets 2 to 8, Coiflets 1 to 5, Biorthogonal 1.1 to 6.8, and discrete approximation of Meyer. As the sEMG signals are composed of the superposition of the activity of individual MUs, each mother 
wavelet available within Wavelet Toolbox was used to decompose sEMG signals into their constituent components.

\subsubsection{Selecting a Wavelet Function}

EMG signals which were collected from three muscles were analyzed by 38 wavelet functions with 5 levels of decomposition for three phases of dynamic contraction during squat task. The wavelets energies were computed using wenergy function in MATLAB. The 5 wavelet functions that represented the highest amount of energy for each subject were highlighted. A sample of energy after EMG decomposition with 38 various wavelet functions is illustrated in Table $4.1(\mathrm{CON})$ and $4.2(\mathrm{OR})$ for muscle 3 during descending phase. 
Table 4.1: Energy of Muscle3 During Descending Phase for One CON Subject

\begin{tabular}{cccccc}
\hline Wavelet Function & Level 1 & Level 2 & Level 3 & Level 4 & Level 5 \\
\hline Haar & 15.120 & 27.529 & 34.690 & 16.591 & 4.856 \\
db2 & 10.220 & 27.896 & 36.518 & 20.949 & 3.197 \\
db3 & 9.785 & 23.660 & 40.100 & 17.346 & 5.518 \\
db4 & 10.502 & 21.962 & 41.368 & 21.702 & 3.459 \\
db5 & 10.049 & 22.521 & 40.100 & 19.809 & 4.189 \\
db6 & 9.176 & 21.826 & 41.574 & 18.809 & 5.300 \\
db7 & 8.046 & 20.627 & 42.055 & 20.300 & 2.800 \\
db8 & 7.761 & 22.592 & 41.346 & 19.390 & 3.732 \\
db9 & 7.774 & 22.194 & 39.522 & 18.620 & 4.799 \\
db10 & 8.444 & 20.238 & 43.060 & 19.488 & 2.459 \\
sym2 & 10.220 & 27.896 & 36.518 & 20.949 & 3.197 \\
sym3 & 9.785 & 23.660 & 40.100 & 17.346 & 5.518 \\
sym4 & 8.891 & 21.050 & 38.883 & 16.105 & 4.991 \\
sym5 & 7.830 & 21.696 & 38.846 & 19.735 & 2.744 \\
sym6 & 8.463 & 22.090 & 38.997 & 16.688 & 5.269 \\
sym7 & 8.564 & 21.338 & 38.200 & 20.650 & 2.364 \\
sym8 & 8.587 & 20.763 & 42.896 & 19.311 & 3.631 \\
coif1 & 11.569 & 23.149 & 36.864 & 16.557 & 5.047 \\
coif2 & 9.166 & 20.632 & 38.418 & 17.805 & 5.534 \\
coif3 & 8.739 & 19.966 & 40.307 & 19.535 & 3.033 \\
coif4 & 8.612 & 19.975 & 43.066 & 20.828 & 2.865 \\
coif5 & 8.436 & 19.785 & 42.044 & 24.403 & 4.077 \\
bior1.1 & 15.120 & 27.529 & 34.690 & 16.591 & 4.856 \\
bior1.3 & 12.044 & 26.592 & 34.413 & 16.518 & 2.682 \\
bior1.5 & 11.064 & 24.338 & 35.020 & 18.426 & 2.460 \\
bior2.2 & 4.824 & 14.425 & 38.522 & 23.018 & 9.213 \\
bior2.4 & 4.767 & 15.283 & 39.407 & 21.715 & 8.427 \\
bior2.6 & 4.633 & 14.8250 & 43.120 & 22.726 & 7.580 \\
bior2.8 & 4.567 & 15.491 & 40.996 & 29.308 & 5.7048 \\
bior3.1 & 0.865 & 4.250 & 20.502 & 19.406 & 18.944 \\
bior3.3 & 1.733 & 8.826 & 36.100 & 30.636 & 7.914 \\
bior3.5 & 1.818 & 8.897 & 43.999 & 32.089 & 6.204 \\
bior3.7 & 1.937 & 9.931 & 43.355 & 32.706 & 7.727 \\
bior3.9 & 1.847 & 9.214 & 46.372 & 31.282 & 9.722 \\
bior4.4 & 8.722 & 20.148 & 38.495 & 17.991 & 5.734 \\
bior5.5 & 14.124 & 24.915 & 34.242 & 13.928 & 4.516 \\
bior6.8 & 7.673 & 19.437 & 39.891 & 24.474 & 4.434 \\
dmey & 7.530 & 18.164 & 43.968 & 24.822 & 3.789 \\
\hline & & & & &
\end{tabular}


Table 4.2: Energy of Muscle3 During Descending Phase for One OR Subject

\begin{tabular}{cccccc}
\hline Wavelet Function & Level 1 & Level 2 & Level 3 & Level 4 & Level 5 \\
\hline Haar & 15.441 & 22.152 & 28.738 & 31.408 & 1.585 \\
db2 & 12.192 & 19.143 & 29.079 & 35.957 & 1.931 \\
db3 & 11.325 & 17.618 & 28.551 & 37.383 & 2.065 \\
db4 & 11.032 & 16.790 & 26.697 & 38.895 & 1.652 \\
db5 & 11.040 & 16.697 & 27.399 & 37.952 & 1.914 \\
db6 & 10.902 & 16.651 & 25.619 & 40.017 & 2.118 \\
db7 & 10.740 & 17.213 & 26.873 & 40.481 & 1.727 \\
db8 & 10.400 & 17.066 & 24.564 & 42.755 & 2.060 \\
db9 & 10.331 & 16.843 & 25.708 & 40.969 & 2.656 \\
db10 & 10.358 & 16.712 & 23.525 & 42.447 & 2.561 \\
sym2 & 12.192 & 19.143 & 29.079 & 35.957 & 1.931 \\
sym3 & 11.325 & 17.618 & 28.551 & 37.383 & 2.065 \\
sym4 & 11.343 & 16.982 & 28.263 & 37.667 & 1.878 \\
sym5 & 10.568 & 17.073 & 26.595 & 37.263 & 1.627 \\
sym6 & 10.480 & 16.350 & 24.787 & 38.049 & 1.668 \\
sym7 & 10.515 & 16.832 & 26.468 & 38.674 & 1.354 \\
sym8 & 10.620 & 17.153 & 24.540 & 40.232 & 1.534 \\
coif1 & 12.308 & 18.091 & 29.336 & 34.931 & 2.393 \\
coif2 & 11.021 & 16.771 & 26.075 & 37.916 & 1.721 \\
coif3 & 10.708 & 16.753 & 26.577 & 39.663 & 1.404 \\
coif4 & 10.492 & 16.805 & 23.920 & 42.391 & 2.870 \\
coif5 & 10.575 & 16.901 & 25.096 & 43.061 & 2.884 \\
bior1.1 & 15.441 & 22.152 & 28.738 & 31.408 & 1.585 \\
bior1.3 & 12.607 & 20.070 & 27.734 & 35.216 & 1.347 \\
bior1.5 & 11.483 & 19.312 & 26.912 & 35.102 & 1.051 \\
bior2.2 & 4.751 & 13.865 & 14.825 & 59.455 & 3.717 \\
bior2.4 & 4.311 & 12.774 & 13.690 & 59.857 & 2.780 \\
bior2.6 & 4.174 & 12.822 & 12.633 & 61.436 & 2.501 \\
bior2.8 & 4.184 & 13.144 & 13.744 & 63.158 & 1.925 \\
bior3.1 & 0.924 & 6.423 & 5.133 & 59.544 & 3.052 \\
bior3.3 & 1.432 & 9.331 & 5.853 & 75.889 & 3.281 \\
bior3.5 & 1.441 & 8.949 & 5.844 & 75.392 & 2.656 \\
bior3.7 & 1.447 & 8.855 & 6.117 & 76.127 & 3.356 \\
bior3.9 & 1.389 & 8.387 & 5.274 & 81.126 & 2.814 \\
bior4.4 & 10.175 & 19.480 & 18.685 & 42.464 & 2.277 \\
bior5.5 & 19.749 & 24.367 & 21.770 & 25.625 & 1.529 \\
bior6.8 & 9.084 & 17.317 & 19.540 & 47.831 & 1.650 \\
dmey & 8.963 & 14.621 & 20.305 & 50.309 & 4.627 \\
\hline & & & & & \\
\hline
\end{tabular}


The highest five energies were indicated in red in Table 4.2. The same scenario was repeated for all subjects with analyzing EMG signals from all three muscles for each descending, stationary, and ascending phase separately. The highest five energies occurred in various levels for different subjects and muscles. The five WFs that indicated the highest percentage of energy were selected for each subject. Table 4.3 and 4.4 show the five selected WFs for one CON and OR subject.

Table 4.3: Five Wavelet Functions With Highest Energy for Muscle 3 During Descending Phase of One CON Subject

\begin{tabular}{cccccc}
\hline Wavelet Function & Level 1 & Level 2 & Level 3 & Level 4 & Level 5 \\
\hline bior2.6 & 4.633 & 14.825 & 43.120 & 22.726 & 7.580 \\
bior3.5 & 1.818 & 8.897 & 43.999 & 32.089 & 6.204 \\
bior3.7 & 1.937 & 9.931 & 43.355 & 32.706 & 7.727 \\
bior3.9 & 1.847 & 9.214 & 46.372 & 31.282 & 9.722 \\
dmey & 7.530 & 18.164 & 43.968 & 24.822 & 3.789 \\
\hline
\end{tabular}

Table 4.4: Five Wavelet Functions With Highest Energy for Muscle 3 During Descending Phase of One OR Subject

\begin{tabular}{cccccc}
\hline Wavelet Function & Level 1 & Level 2 & Level 3 & Level 4 & Level 5 \\
\hline bior2.8 & 4.184 & 13.144 & 13.743 & 63.158 & 1.925 \\
bior3.3 & 1.432 & 9.331 & 5.853 & 75.889 & 3.281 \\
bior3.5 & 1.441 & 8.949 & 5.844 & 75.392 & 2.656 \\
bior3.7 & 1.447 & 8.855 & 6.117 & 76.127 & 3.356 \\
bior3.9 & 1.389 & 8.387 & 5.274 & 81.126 & 2.814 \\
\hline
\end{tabular}

The total number of repetition of wavelet functions between CON and OR group during descending, stationary, and ascending phases were computed for each muscle separately. Based on our observation (Tables 4.5 - 4.10) there is not a single wavelet function that gives the best contrast between all subjects. 
Table 4.5: Selection of a WF With Highest Repetition of Energy for Muscle 1-CON

\begin{tabular}{ccccc}
\hline Wavelet Function & Descending & Stationary & Ascending & Total \\
\hline Haar & 1 & 3 & 0 & 4 \\
db2 & 0 & 0 & 0 & 0 \\
db3 & 1 & 1 & 0 & 2 \\
db4 & 1 & 2 & 3 & 6 \\
db5 & 1 & 1 & 4 & 6 \\
db6 & 2 & 4 & 1 & 7 \\
db7 & 0 & 2 & 3 & 5 \\
db8 & 3 & 2 & 5 & 10 \\
db9 & 1 & 2 & 3 & 6 \\
db10 & 2 & 2 & 6 & 10 \\
sym2 & 1 & 1 & 0 & 2 \\
sym3 & 1 & 1 & 0 & 2 \\
sym4 & 1 & 1 & 0 & 2 \\
sym5 & 3 & 0 & 2 & 5 \\
sym6 & 0 & 1 & 2 & 3 \\
sym7 & 0 & 1 & 3 & 4 \\
sym8 & 1 & 0 & 3 & 4 \\
coif1 & 0 & 0 & 0 & 0 \\
coif2 & 0 & 0 & 1 & 1 \\
coif3 & 0 & 3 & 1 & 4 \\
coif4 & 1 & 0 & 2 & 3 \\
coif5 & 3 & 3 & 3 & 9 \\
bior1.1 & 1 & 3 & 0 & 4 \\
bior1.3 & 1 & 0 & 0 & 1 \\
bior1.5 & 1 & 0 & 0 & 1 \\
bior2.2 & 1 & 0 & 1 & 2 \\
bior2.4 & 2 & 2 & 2 & 6 \\
bior2.6 & 2 & 2 & 4 & 8 \\
bior2.8 & 5 & 5 & 6 & 16 \\
bior3.1 & 0 & 1 & 0 & 1 \\
bior3.3 & 7 & 6 & 0 & 13 \\
bior3.5 & 6 & 5 & 3 & 14 \\
bior3.7 & 7 & 6 & 4 & 17 \\
bior3.9 & 12 & 6 & 7 & 25 \\
bior4.4 & 1 & 1 & 1 & 3 \\
bior5.5 & 1 & 0 & 5 \\
bior6.8 & 9 & 3 & 8 \\
dmey & & 7 & 20 \\
\hline & & & \\
\hline
\end{tabular}


Table 4.6: Selection of a WF With Highest Repetition of Energy for Muscle 1-OR

\begin{tabular}{ccccc}
\hline Wavelet Function & Descending & Stationary & Ascending & Total \\
\hline Haar & 1 & 4 & 0 & 5 \\
db2 & 0 & 0 & 0 & 0 \\
db3 & 1 & 1 & 1 & 3 \\
db4 & 1 & 3 & 3 & 7 \\
db5 & 3 & 1 & 1 & 5 \\
db6 & 1 & 0 & 4 & 5 \\
db7 & 0 & 2 & 4 & 6 \\
db8 & 3 & 3 & 4 & 10 \\
db9 & 2 & 2 & 3 & 7 \\
db10 & 3 & 2 & 3 & 8 \\
sym2 & 0 & 1 & 0 & 1 \\
sym3 & 1 & 1 & 1 & 3 \\
sym4 & 2 & 1 & 1 & 4 \\
sym5 & 2 & 0 & 2 & 4 \\
sym6 & 1 & 0 & 1 & 2 \\
sym7 & 0 & 1 & 3 & 4 \\
sym8 & 1 & 0 & 2 & 3 \\
coif1 & 1 & 1 & 0 & 2 \\
coif2 & 0 & 0 & 1 & 1 \\
coif3 & 1 & 0 & 2 & 3 \\
coif4 & 2 & 1 & 3 & 6 \\
coif5 & 2 & 2 & 2 & 6 \\
bior1.1 & 1 & 4 & 0 & 5 \\
bior1.3 & 0 & 0 & 0 & 0 \\
bior1.5 & 0 & 0 & 0 & 0 \\
bior2.2 & 2 & 1 & 1 & 4 \\
bior2.4 & 1 & 0 & 1 & 2 \\
bior2.6 & 0 & 0 & 1 & 1 \\
bior2.8 & 0 & 1 & 2 & 3 \\
bior3.1 & 1 & 0 & 0 & 1 \\
bior3.3 & 4 & 2 & 0 & 6 \\
bior3.5 & 6 & 3 & 1 & 10 \\
bior3.7 & 6 & 6 & 4 & 16 \\
bior3.9 & 4 & 6 & 4 & 14 \\
bior4.4 & 0 & 1 & 0 & 1 \\
bior5.5 & 3 & 0 & 0 & 3 \\
bior6.8 & 0 & 1 & 1 & 2 \\
dmey & 3 & 5 & 15 \\
\hline & & & & \\
\hline
\end{tabular}


Table 4.7: Selection of a WF With Highest Repetition of Energy for Muscle 2-CON

\begin{tabular}{ccccc}
\hline Wavelet Function & Descending & Stationary & Ascending & Total \\
\hline Haar & 0 & 3 & 0 & 3 \\
db2 & 0 & 0 & 1 & 1 \\
db3 & 1 & 2 & 3 & 6 \\
db4 & 3 & 0 & 2 & 5 \\
db5 & 1 & 3 & 3 & 7 \\
db6 & 1 & 1 & 1 & 3 \\
db7 & 5 & 1 & 4 & 10 \\
db8 & 2 & 1 & 5 & 8 \\
db9 & 6 & 1 & 4 & 11 \\
db10 & 2 & 2 & 0 & 4 \\
sym2 & 2 & 3 & 2 & 7 \\
sym3 & 1 & 2 & 3 & 6 \\
sym4 & 2 & 1 & 3 & 6 \\
sym5 & 0 & 1 & 3 & 4 \\
sym6 & 1 & 1 & 0 & 2 \\
sym7 & 2 & 1 & 2 & 5 \\
sym8 & 1 & 1 & 2 & 4 \\
coif1 & 0 & 1 & 1 & 2 \\
coif2 & 0 & 1 & 0 & 1 \\
coif3 & 3 & 1 & 3 & 7 \\
coif4 & 0 & 0 & 3 & 3 \\
coif5 & 6 & 6 & 4 & 16 \\
bior1.1 & 0 & 3 & 0 & 3 \\
bior1.3 & 2 & 0 & 0 & 2 \\
bior1.5 & 0 & 0 & 0 & 0 \\
bior2.2 & 1 & 2 & 2 & 5 \\
bior2.4 & 2 & 1 & 1 & 4 \\
bior2.6 & 2 & 1 & 0 & 3 \\
bior2.8 & 3 & 4 & 5 & 12 \\
bior3.1 & 0 & 0 & 0 & 0 \\
bior3.3 & 3 & 5 & 3 & 11 \\
bior3.5 & 6 & 6 & 4 & 16 \\
bior3.7 & 7 & 3 & 4 & 14 \\
bior3.9 & 8 & 8 & 4 & 20 \\
bior4.4 & 0 & 0 & 0 & 0 \\
bior5.5 & 0 & 1 & 4 & 6 \\
bior6.8 & 1 & & 18 \\
dmey & & & \\
\hline & & & \\
\hline
\end{tabular}


Table 4.8: Selection of a WF With Highest Repetition of Energy for Muscle 2-OR

\begin{tabular}{ccccc}
\hline Wavelet Function & Descending & Stationary & Ascending & Total \\
\hline Haar & 0 & 2 & 0 & 2 \\
db2 & 0 & 0 & 0 & 0 \\
db3 & 0 & 2 & 1 & 3 \\
db4 & 1 & 3 & 1 & 5 \\
db5 & 3 & 1 & 2 & 6 \\
db6 & 3 & 1 & 2 & 6 \\
db7 & 1 & 2 & 0 & 3 \\
db8 & 3 & 2 & 3 & 8 \\
db9 & 2 & 2 & 2 & 6 \\
db10 & 1 & 1 & 1 & 3 \\
sym2 & 1 & 1 & 0 & 2 \\
sym3 & 0 & 2 & 1 & 3 \\
sym4 & 1 & 1 & 2 & 4 \\
sym5 & 1 & 1 & 2 & 4 \\
sym6 & 0 & 0 & 0 & 0 \\
sym7 & 1 & 1 & 2 & 4 \\
sym8 & 2 & 0 & 1 & 3 \\
coif1 & 0 & 0 & 1 & 1 \\
coif2 & 0 & 0 & 0 & 0 \\
coif3 & 0 & 2 & 1 & 3 \\
coif4 & 1 & 0 & 1 & 2 \\
coif5 & 0 & 5 & 3 & 8 \\
bior1.1 & 0 & 2 & 0 & 2 \\
bior1.3 & 0 & 0 & 0 & 0 \\
bior1.5 & 0 & 1 & 0 & 1 \\
bior2.2 & 2 & 0 & 3 & 5 \\
bior2.4 & 3 & 1 & 2 & 6 \\
bior2.6 & 1 & 0 & 2 & 3 \\
bior2.8 & 5 & 6 & 5 & 16 \\
bior3.1 & 0 & 0 & 0 & 0 \\
bior3.3 & 1 & 2 & 1 & 4 \\
bior3.5 & 7 & 2 & 6 & 15 \\
bior3.7 & 8 & 3 & 6 & 17 \\
bior3.9 & 9 & 7 & 9 & 25 \\
bior4.4 & 0 & 0 & 0 & 0 \\
bior5.5 & 0 & 0 & 1 & 1 \\
bior6.8 & 3 & 3 & 3 & 9 \\
dmey & & & 14 \\
\hline & & & \\
\hline
\end{tabular}


Table 4.9: Selection of a WF With Highest Repetition of Energy for Muscle 3-CON

\begin{tabular}{|c|c|c|c|c|}
\hline Wavelet Function & Descending & Stationary & Ascending & Total \\
\hline Haar & 0 & 4 & 0 & 4 \\
\hline $\mathrm{db} 2$ & 0 & 2 & 0 & 2 \\
\hline db3 & 1 & 1 & 0 & 2 \\
\hline $\mathrm{db} 4$ & 2 & 2 & 2 & 6 \\
\hline db5 & 1 & 0 & 1 & 2 \\
\hline db6 & 1 & 1 & 1 & 3 \\
\hline $\mathrm{db} 7$ & 5 & 1 & 2 & 8 \\
\hline $\mathrm{db} 8$ & 4 & 0 & 4 & 8 \\
\hline db9 & 3 & 2 & 2 & 7 \\
\hline db10 & 7 & 2 & 3 & 12 \\
\hline sym2 & 0 & 6 & 1 & 7 \\
\hline sym3 & 1 & 1 & 0 & 2 \\
\hline sym4 & 1 & 0 & 0 & 1 \\
\hline sym5 & 0 & 1 & 1 & 2 \\
\hline sym6 & 2 & 1 & 0 & 3 \\
\hline sym7 & 2 & 1 & 3 & 6 \\
\hline sym8 & 2 & 3 & 6 & 11 \\
\hline coif1 & 1 & 0 & 0 & 1 \\
\hline coif2 & 3 & 0 & 0 & 3 \\
\hline coif3 & 0 & 1 & 3 & 4 \\
\hline coif4 & 4 & 3 & 5 & 12 \\
\hline coif5 & 2 & 5 & 2 & 9 \\
\hline bior1.1 & 0 & 4 & 0 & 4 \\
\hline bior1.3 & 0 & 1 & 2 & 3 \\
\hline bior1.5 & 0 & 1 & 0 & 1 \\
\hline bior2.2 & 1 & 0 & 1 & 2 \\
\hline bior2.4 & 3 & 0 & 2 & 5 \\
\hline bior2.6 & 4 & 1 & 0 & 5 \\
\hline bior2.8 & 2 & 2 & 6 & 10 \\
\hline bior3.1 & 0 & 0 & 0 & 0 \\
\hline bior3.3 & 0 & 2 & 0 & 2 \\
\hline bior3.5 & 8 & 5 & 2 & 15 \\
\hline bior3.7 & 8 & 3 & 11 & 22 \\
\hline bior3.9 & 7 & 8 & 13 & 28 \\
\hline bior4.4 & 2 & 0 & 0 & 2 \\
\hline bior5.5 & 0 & 1 & 0 & 1 \\
\hline bior6.8 & 1 & 0 & 2 & 3 \\
\hline dmey & 2 & 11 & 4 & 17 \\
\hline
\end{tabular}


Table 4.10: Selection of a WF With Highest Repetition of Energy for Muscle 3-OR

\begin{tabular}{ccccc}
\hline Wavelet Function & Descending & Stationary & Ascending & Total \\
\hline Haar & 0 & 4 & 0 & 4 \\
db2 & 0 & 0 & 0 & 0 \\
db3 & 0 & 2 & 0 & 2 \\
db4 & 0 & 1 & 0 & 1 \\
db5 & 0 & 2 & 0 & 2 \\
db6 & 3 & 2 & 1 & 6 \\
db7 & 2 & 2 & 1 & 5 \\
db8 & 1 & 1 & 2 & 4 \\
db9 & 3 & 2 & 3 & 8 \\
db10 & 2 & 1 & 1 & 4 \\
sym2 & 0 & 0 & 0 & 0 \\
sym3 & 0 & 2 & 0 & 2 \\
sym4 & 0 & 0 & 1 & 1 \\
sym5 & 3 & 0 & 1 & 4 \\
sym6 & 1 & 0 & 2 & 3 \\
sym7 & 2 & 1 & 2 & 5 \\
sym8 & 2 & 2 & 3 & 7 \\
coif1 & 0 & 0 & 1 & 1 \\
coif2 & 0 & 0 & 1 & 1 \\
coif3 & 0 & 2 & 1 & 3 \\
coif4 & 3 & 1 & 2 & 6 \\
coif5 & 0 & 5 & 2 & 7 \\
bior1.1 & 0 & 4 & 0 & 4 \\
bior1.3 & 0 & 0 & 0 & 0 \\
bior1.5 & 0 & 0 & 0 & 0 \\
bior2.2 & 0 & 1 & 3 & 4 \\
bior2.4 & 0 & 1 & 2 & 3 \\
bior2.6 & 3 & 1 & 3 & 7 \\
bior2.8 & 7 & 2 & 5 & 14 \\
bior3.1 & 0 & 1 & 0 & 1 \\
bior3.3 & 2 & 0 & 3 & 5 \\
bior3.5 & 7 & 2 & 8 & 17 \\
bior3.7 & 6 & 6 & 6 & 18 \\
bior3.9 & 7 & 10 & 6 & 23 \\
bior4.4 & 2 & 1 & 0 & 3 \\
bior5.5 & 2 & 0 & 3 \\
bior6.8 & 5 & 2 & 7 \\
dmey & & 3 & 11 \\
\hline & & & \\
\hline
\end{tabular}


Bior3.9 wavelet function indicated the highest number of repetitions between subjects for each muscle during three phases except for muscle 1 of OR group which Bior3.7 worked best (Table 4.6). As the difference between Bior3.7 and Bior3.9 was not significant in muscle 1 of OR group, Bior3.9 wavelet function was used for further analyses.

\subsubsection{Principal Component Analysis}

An energy matrix was created from Bior3.9 and the Principal Component Analysis (PCA) method was applied on it. PCA is a data analysis method which involves a mathematical procedure that converts a number of correlated variables into a number of uncorrelated variables called principal components (PCs). The goal of using PCA is to reduce the dimensionality of the data set to identify new meaningful underlying variables. The PCA is a $n \times p$ matrix that has $n$ observations and $p$ variables. The PCA function was used for a 2D matrix and applied to the Bior3.9 energy matrix in order to discriminate two groups of subjects. This function returns a $p \times p$ matrix of coefficients that each column was related to one of our PCs (column one for $P C_{1}$ and column two for $\mathrm{PC}_{2}$ ). Furthermore, the PCA function prepared a SCORE matrix which represents the energy matrix in the PC space. Each column of SCORE corresponds to one PC. Plotting $P C_{1}$ versus $P C_{2}$ did not assess us to discriminate two groups. 


\subsubsection{Bior3.9 Energy and Weight}

Bior3.9 energy for descending, stationary, and ascending phases was plotted for each muscle versus the weight of each subjects. Figures 4.7, 4.8, 4.9 represent the energy versus weight for muscle 1 during squat cycles. Figures 4.10, 4.11, 4.12 show for muscle 2 and Figures 4.13, 4.14, 4.15 indicate the graphs for muscle 3. By referring to these graphs the two groups were not distinguishable based on their energy (both groups indicated almost the same amount of energies). Furthermore, the weight of subjects was not a good parameter to separate them. 


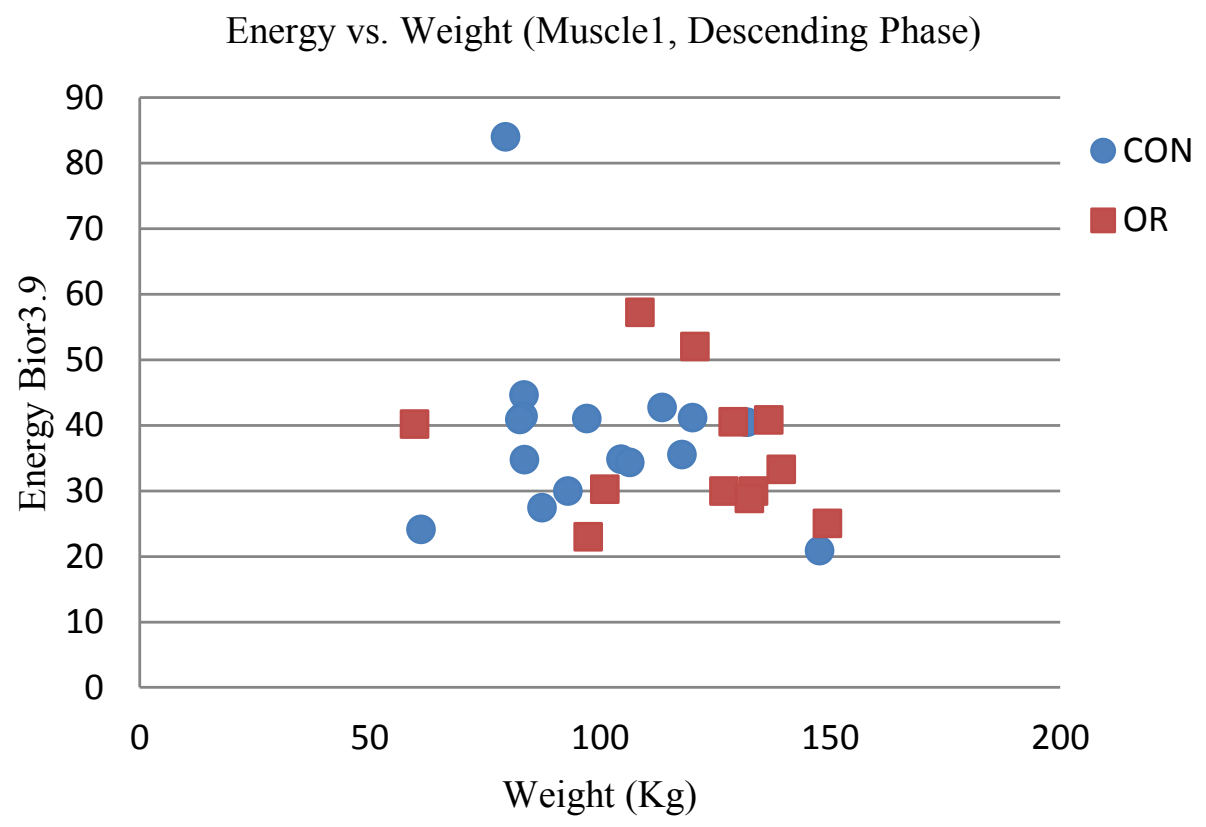

Figure 4.7: Bior3.9 Energy versus Weight for Muscle 1 in Descending Phase 


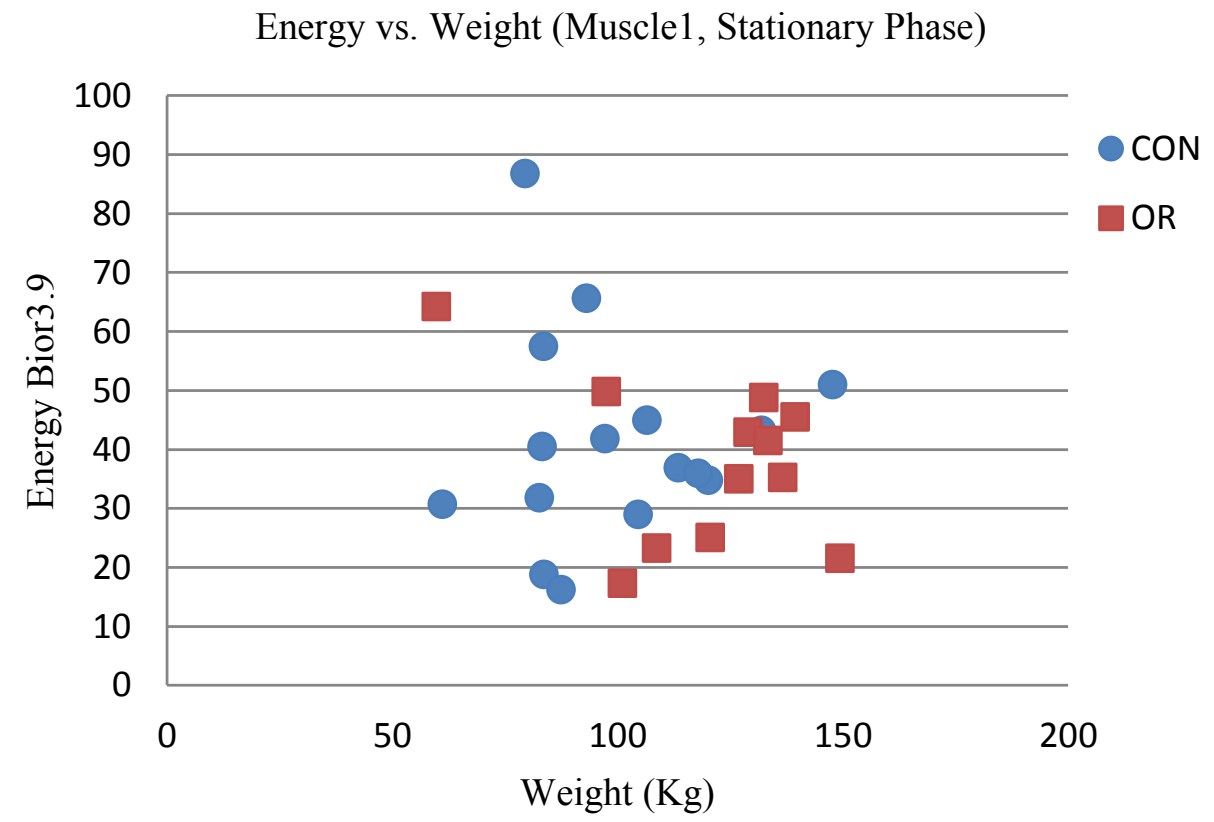

Figure 4.8: Bior3.9 Energy versus Weight for Muscle 1 in Stationary Phase 


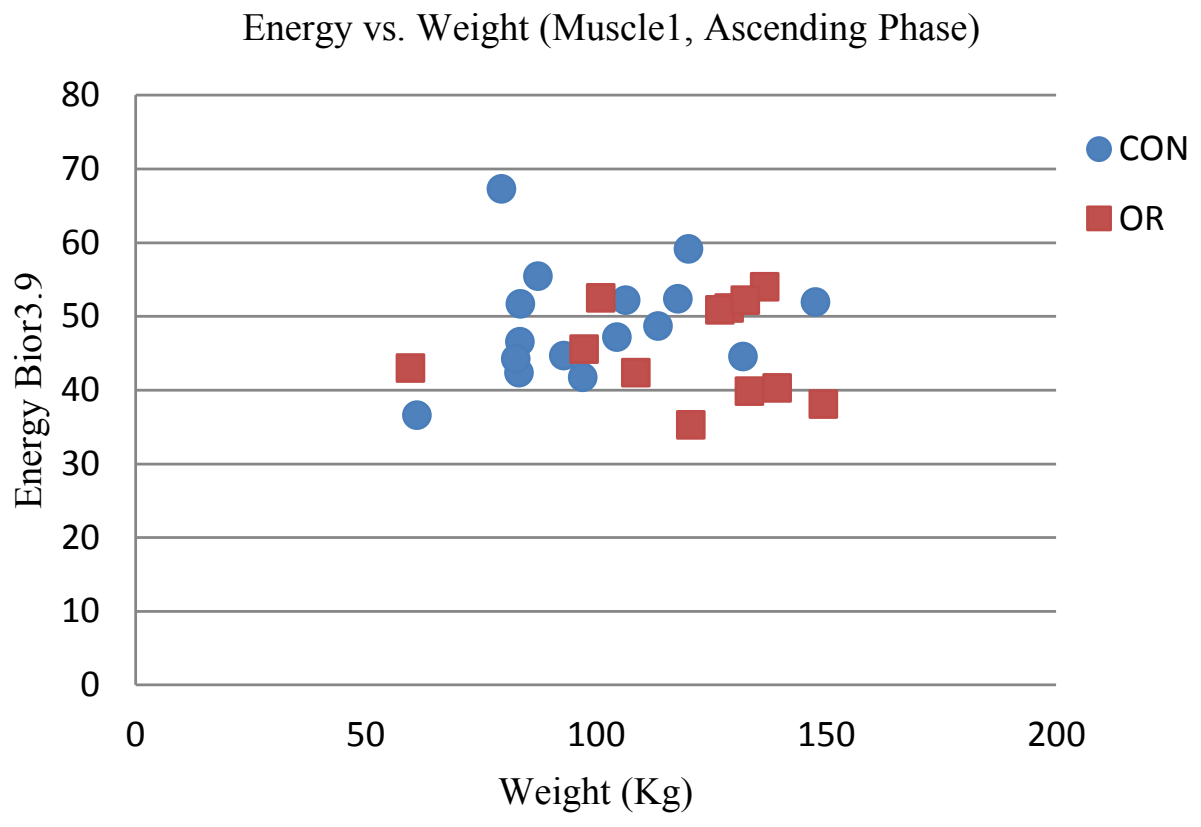

Figure 4.9: Bior3.9 Energy versus Weight for Muscle 1 in Ascending Phase 


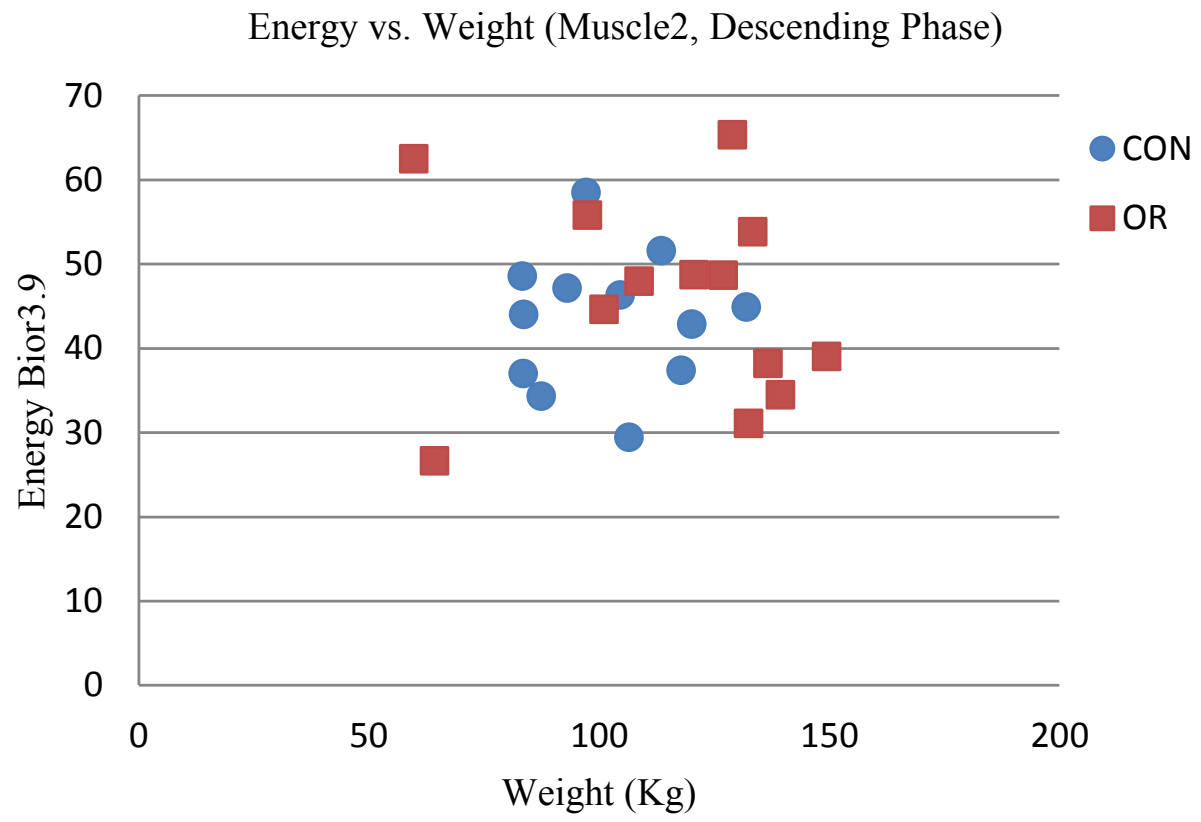

Figure 4.10: Bior3.9 Energy versus Weight for Muscle 2 in Descending Phase 


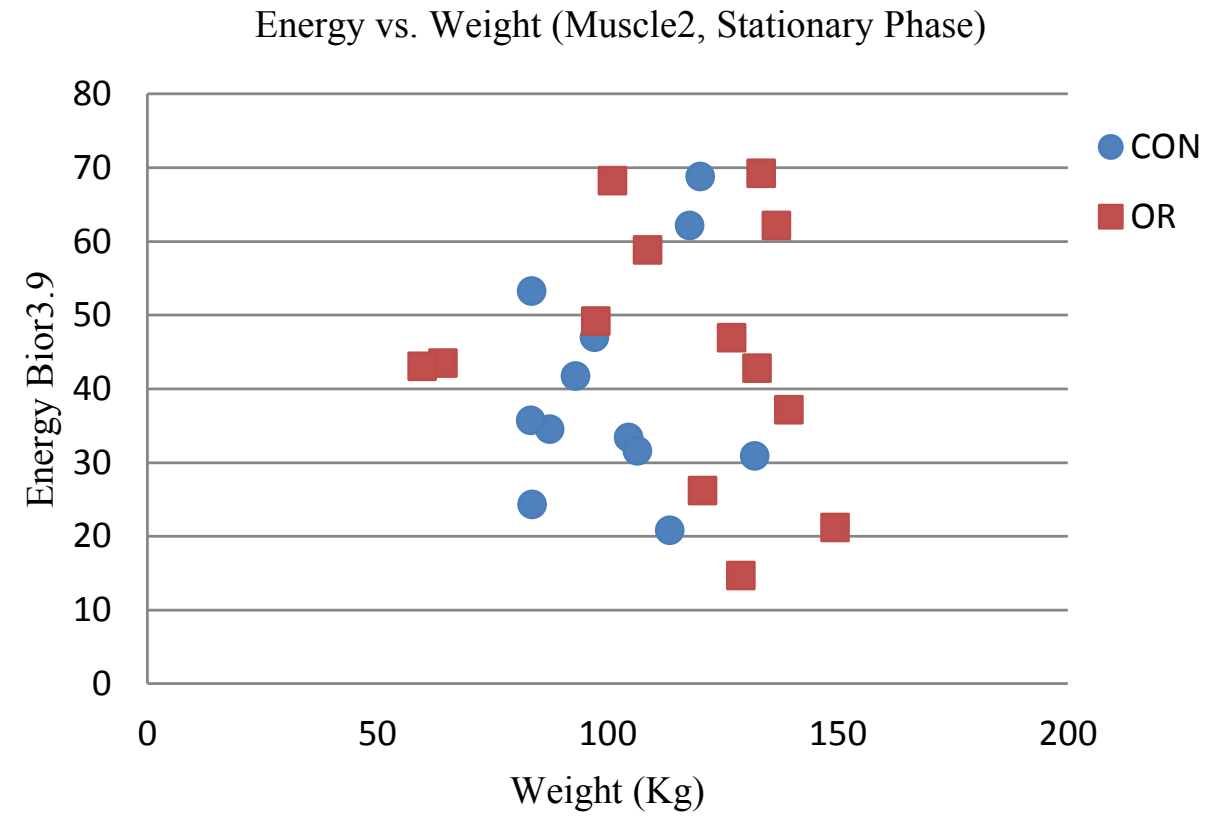

Figure 4.11: Bior3.9 Energy versus Weight for Muscle 2 in Stationary Phase 
Energy vs. weight (Muscle2, Ascending Phase)

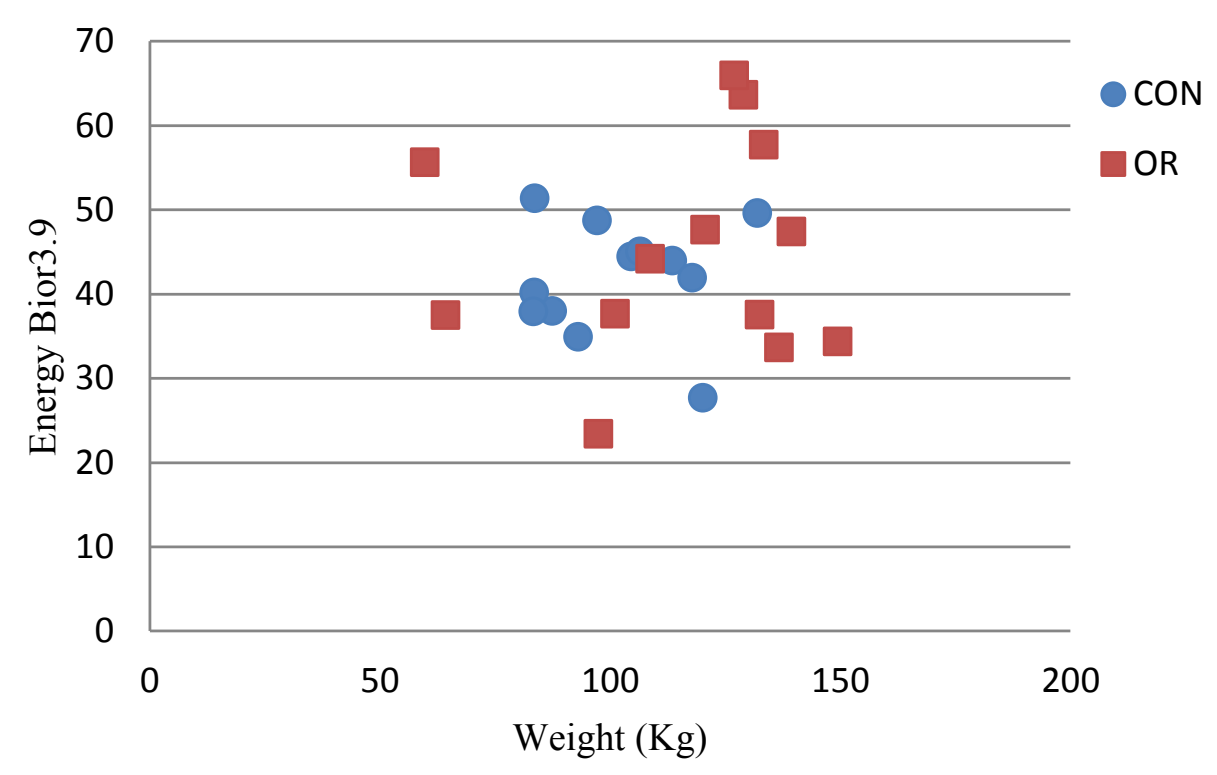

Figure 4.12: Bior3.9 Energy versus Weight for Muscle 2 in Ascending Phase 


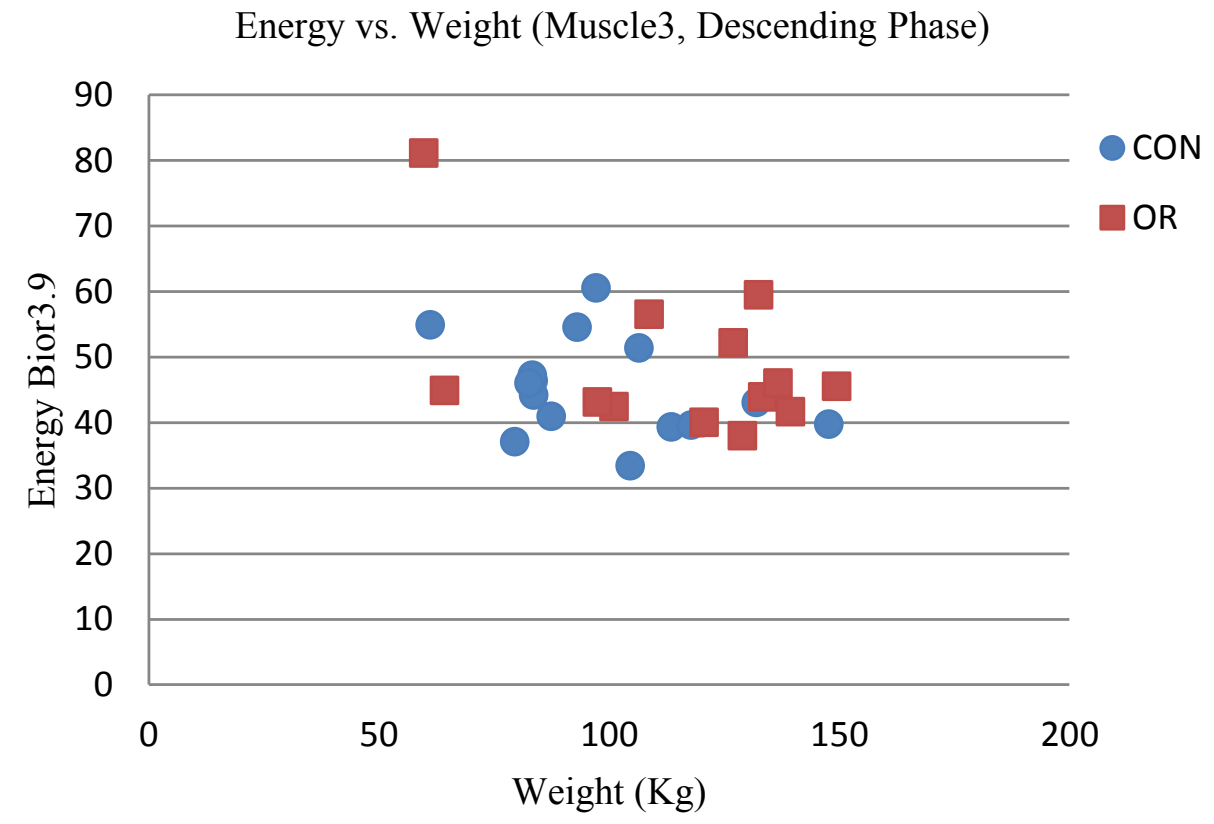

Figure 4.13: Bior3.9 Energy versus Weight for Muscle 3 in Descending Phase 


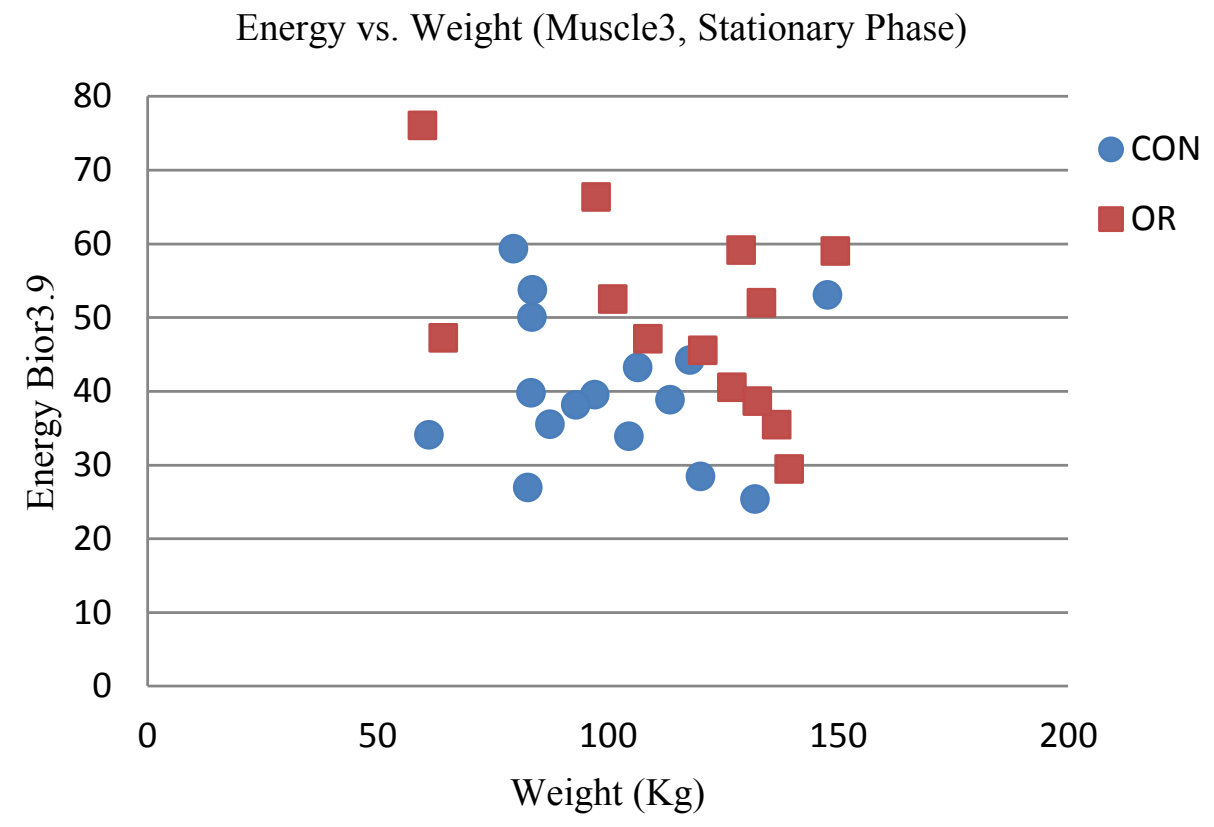

Figure 4.14: Bior3.9 Energy versus Weight for Muscle 3 in Stationary Phase 


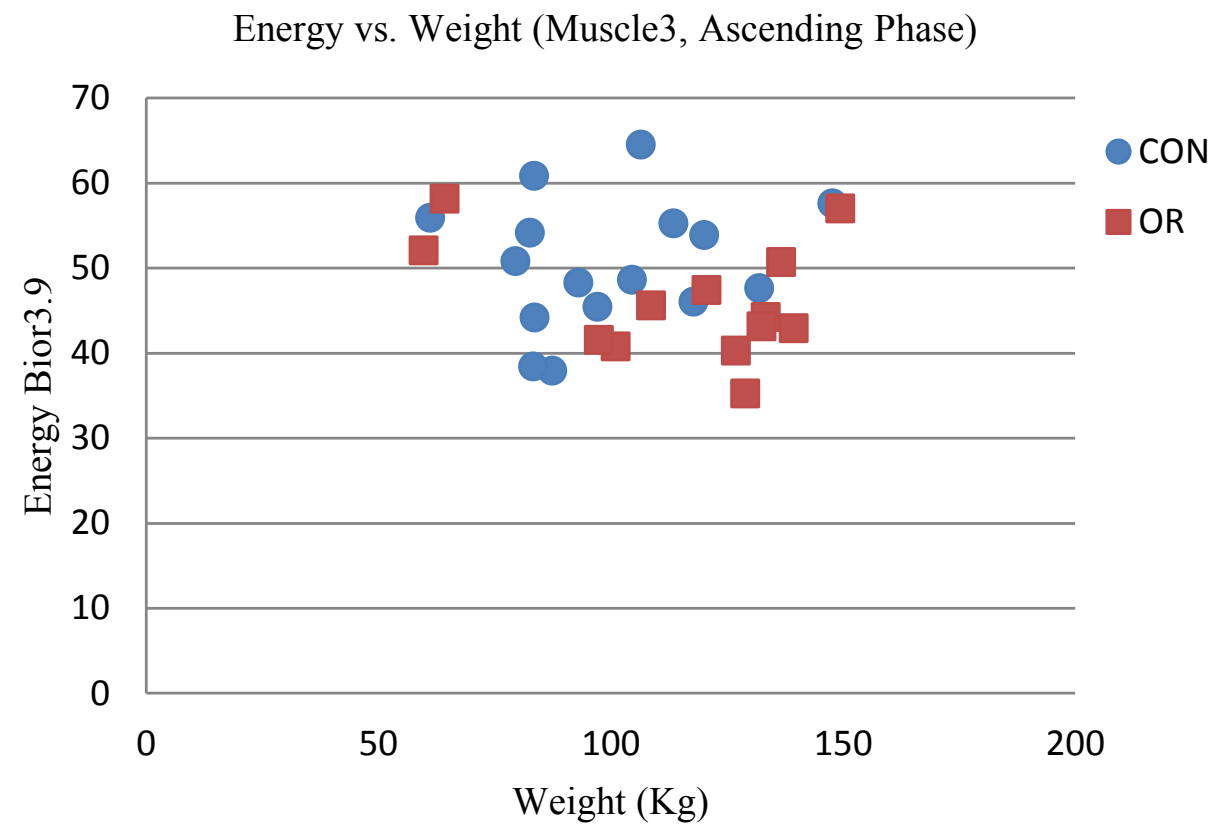

Figure 4.15: Bior3.9 Energy versus Weight for Muscle 3 in Ascending Phase

\subsubsection{Average Energy}

The highest amount of energy for each subject during descending, stationary, and ascending phases was computed (appendix A). Then, the average energy which is the summation of energy from three phases divided by 3 (squat phases) was calculated and plotted in Figure 4.16. 

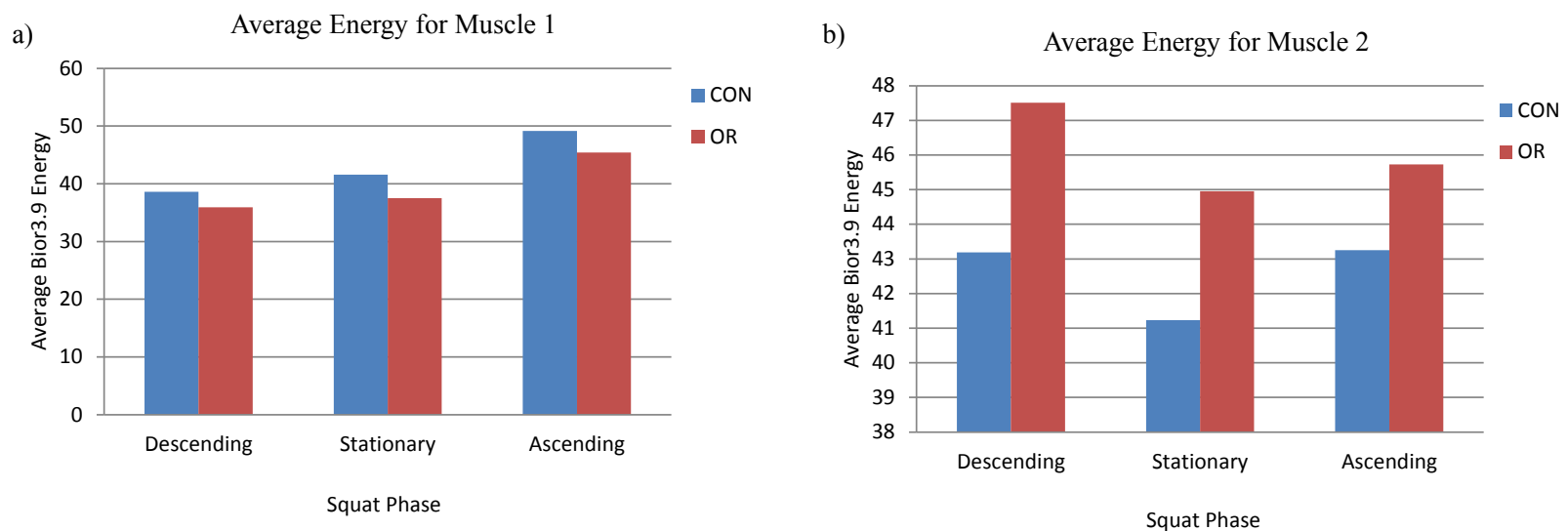

c)

Average Energy for Muscle 3

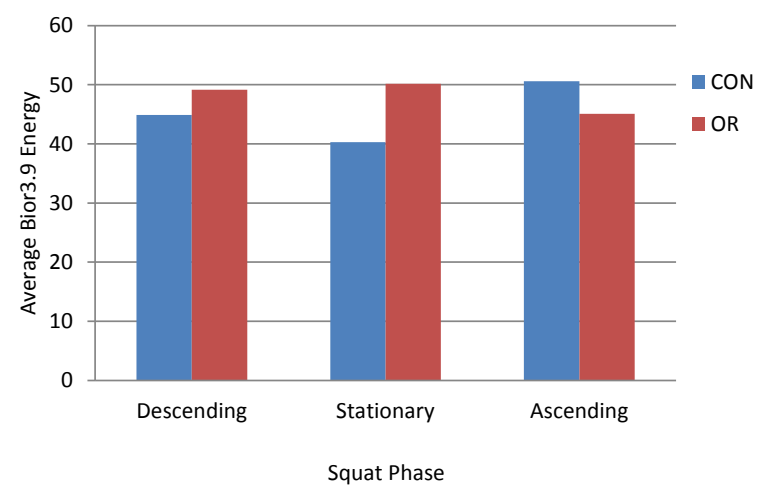

Figure 4.16: Average Energy for Muscle 1, 2, and 3 


\subsubsection{Wavelet Power Spectrum}

The WT converts the data array, which is stored from the EMG recorded signal, into a series of wavelet coefficients. Each of these coefficients represents the amplitude of the wavelet function at a specific location in the array. The best way to specify the distribution of energy within the data is to compute the wavelet power. The power, which is the squared absolute value of the wavelet coefficients, calculated as

$$
W P=\left|W C_{i}\right|^{2}
$$

where $W C$ is wavelet coefficients and $i$ is the index for it.

Therefore, we compute the wavelet power spectrum for each muscle during descending, stationary, and ascending phases by using Bior3.9 WF. Tables 4.11, 4.12, and 4.13 illustrate a sample of calculated wavelet Bior3.9 power after 5 decomposition levels for one CON subject.

Table 4.11: Example of Wavelet Power Spectrum Calculations for Muscle 1 of One CON Subject

\begin{tabular}{cccccc}
\hline & Level 1 & Level 2 & Level 3 & Level 4 & Level 5 \\
\hline Descending (D) & 5.887 & 7.406 & 9.834 & 12.364 & 12.442 \\
Stationary (S) & 0.669 & 1.131 & 1.449 & 1.899 & 2.529 \\
Ascending (A) & 42.010 & 59.763 & 104.155 & 111.192 & 112.685
\end{tabular}


Table 4.12: Example of Wavelet Power Spectrum Calculations for Muscle 2 of One CON Subject

\begin{tabular}{cccccc}
\hline & Level 1 & Level 2 & Level 3 & Level 4 & Level 5 \\
\hline Descending (D) & 20.990 & 26.399 & 35.836 & 46.450 & 39.715 \\
Stationary (S) & 0.025 & 0.043 & 0.058 & 0.078 & 0.104 \\
Ascending (A) & 2.244 & 2.593 & 3.320 & 4.911 & 3.860 \\
\hline
\end{tabular}

Table 4.13: Example of Wavelet Power Spectrum Calculations for Muscle 3 of One CON Subject

\begin{tabular}{cccccc}
\hline & Level 1 & Level 2 & Level 3 & Level 4 & Level 5 \\
\hline Descending (D) & 326.453 & 534.807 & 644.757 & 637.505 & 632.421 \\
Stationary (S) & 48.383 & 95.583 & 112.166 & 117.314 & 129.691 \\
Ascending (A) & 91.764 & 155.638 & 167.959 & 166.553 & 168.692 \\
\hline
\end{tabular}

Furthermore, Tables $4.14,4.15$, and 4.16 show a sample of power calculation for one OR subject (refer to the wavelet power spectrum section in appendix A for power calculation for other subjects).

Table 4.14: Example of Wavelet Power Spectrum Calculations for Muscle 1 of One OR Subject

\begin{tabular}{cccccc}
\hline & Level 1 & Level 2 & Level 3 & Level 4 & Level 5 \\
\hline Descending (D) & 4.897 & 6.234 & 7.728 & 8.162 & 8.667 \\
Stationary (S) & 1.083 & 1.525 & 3.229 & 4.137 & 4.935 \\
Ascending (A) & 220.683 & 282.050 & 495.946 & 604.871 & 578.224 \\
\hline
\end{tabular}


Table 4.15: Example of Wavelet Power Spectrum Calculations for Muscle 2 of one OR Subject

\begin{tabular}{cccccc}
\hline & Level 1 & Level 2 & Level 3 & Level 4 & Level 5 \\
\hline Descending (D) & 10.424 & 15.486 & 19.268 & 20.263 & 22.215 \\
Stationary (S) & 3.239 & 5.794 & 6.606 & 8.119 & 8.875 \\
Ascending (A) & 43.230 & 60.027 & 73.495 & 73.003 & 72.547 \\
\hline
\end{tabular}

Table 4.16: Example of Wavelet Power Spectrum Calculations for Muscle 3 of One OR Subject

\begin{tabular}{cccccc}
\hline & Level 1 & Level 2 & Level 3 & Level 4 & Level 5 \\
\hline Descending (D) & 209.650 & 356.795 & 366.922 & 351.304 & 350.055 \\
Stationary (S) & 65.453 & 135.278 & 210.988 & 261.701 & 292.567 \\
Ascending (A) & 400.546 & 605.973 & 723.068 & 818.077 & 823.966
\end{tabular}

Then the power after 5 decomposition levels was plotted separately for OR and CON subjects during squat phases. Figures 4.17- 4.19 represent a sample of the wavelet power values for muscle 1,2 , and 3 of a CON subject. 


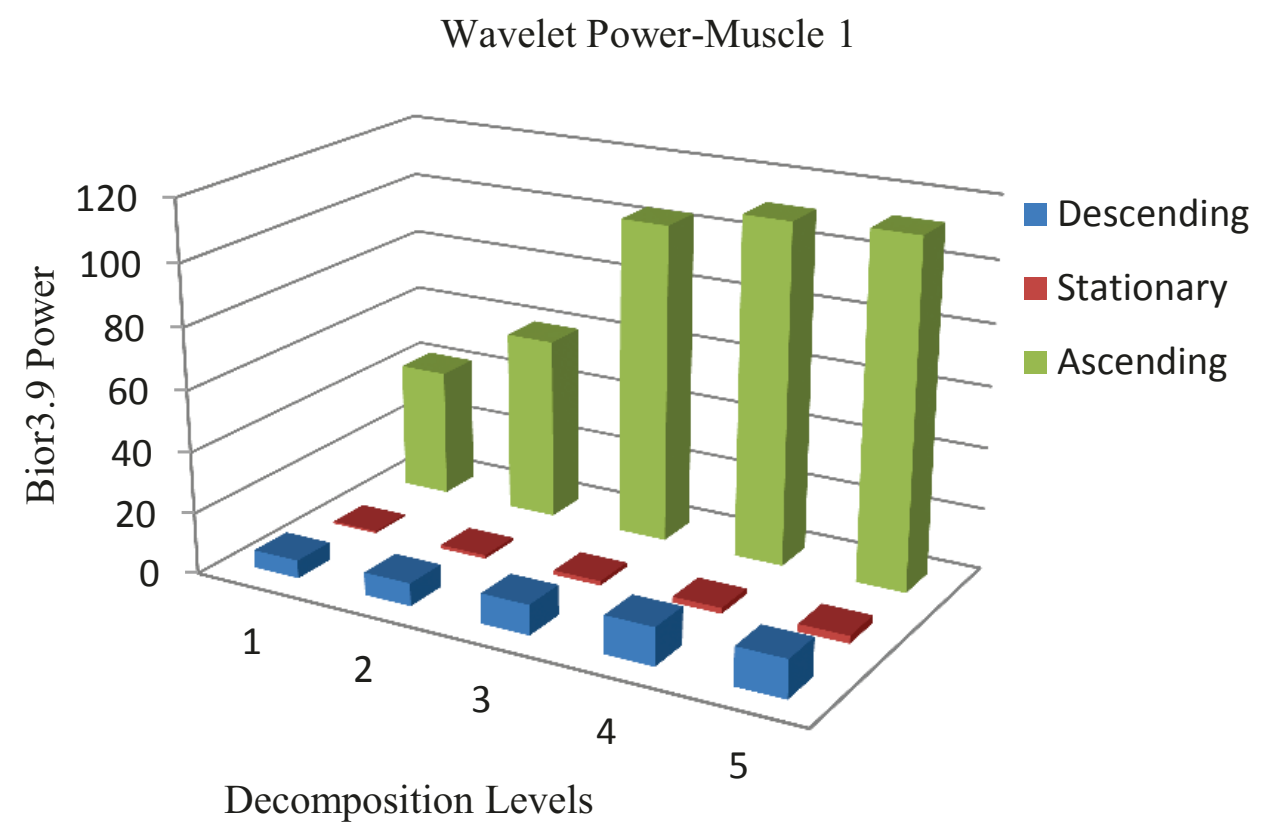

Figure 4.17: Example of Wavelet Power for Muscle 1 of One CON Subject 


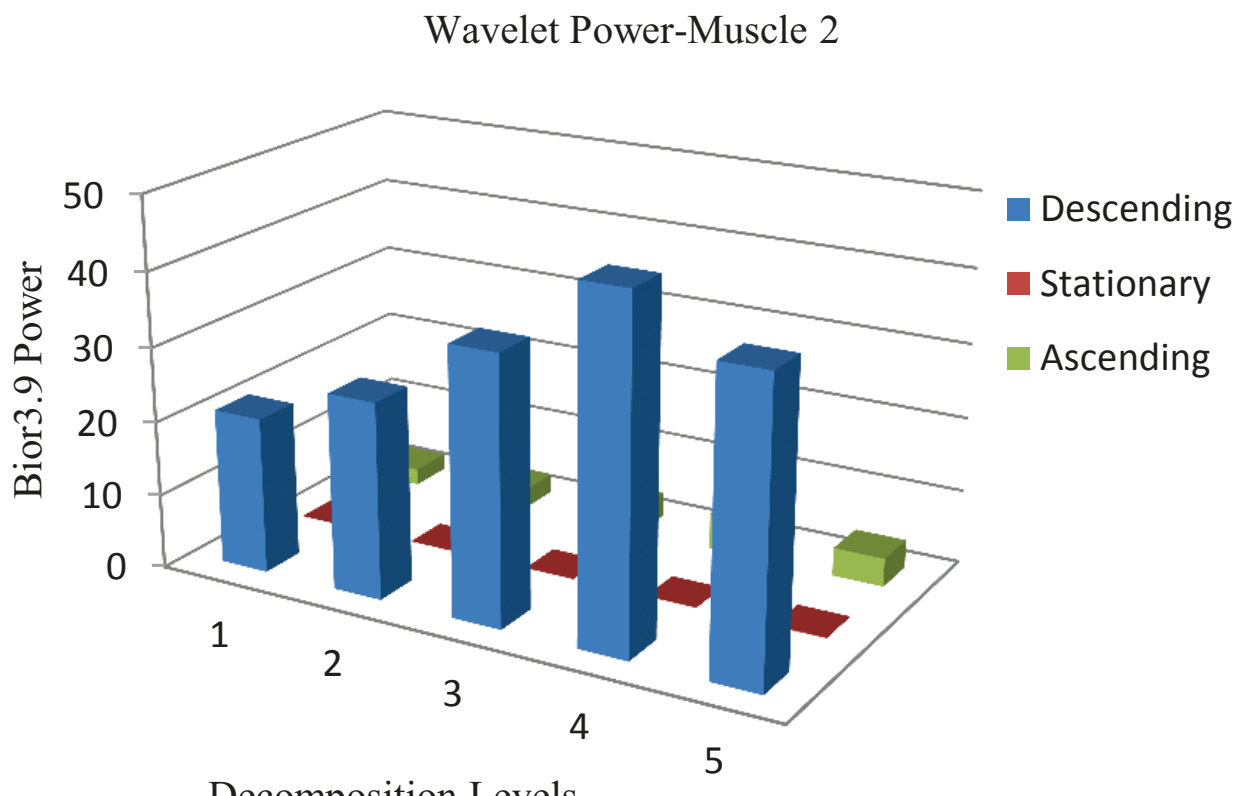

Decomposition Levels

Figure 4.18: Example of Wavelet Power for Muscle 2 of One CON Subject 


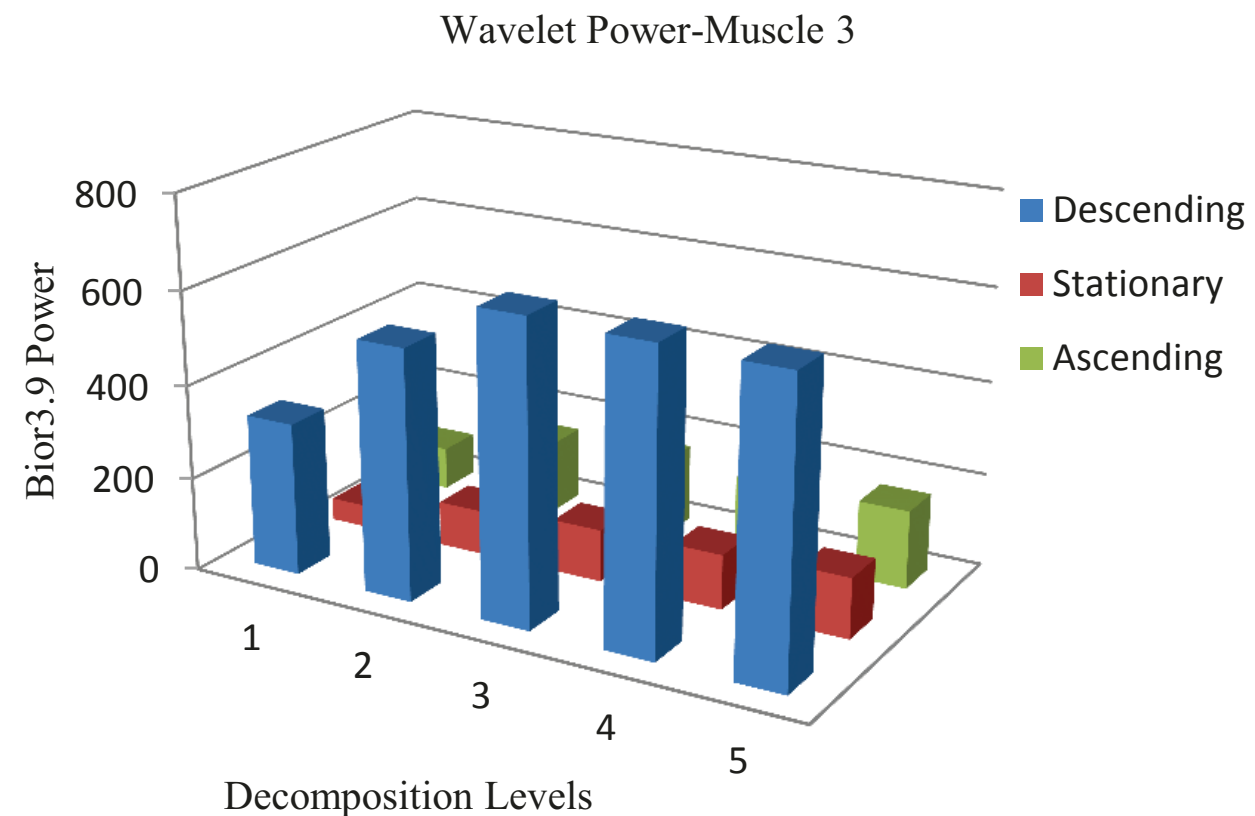

Figure 4.19: Example of Wavelet Power for Muscle 3 of One CON Subject 


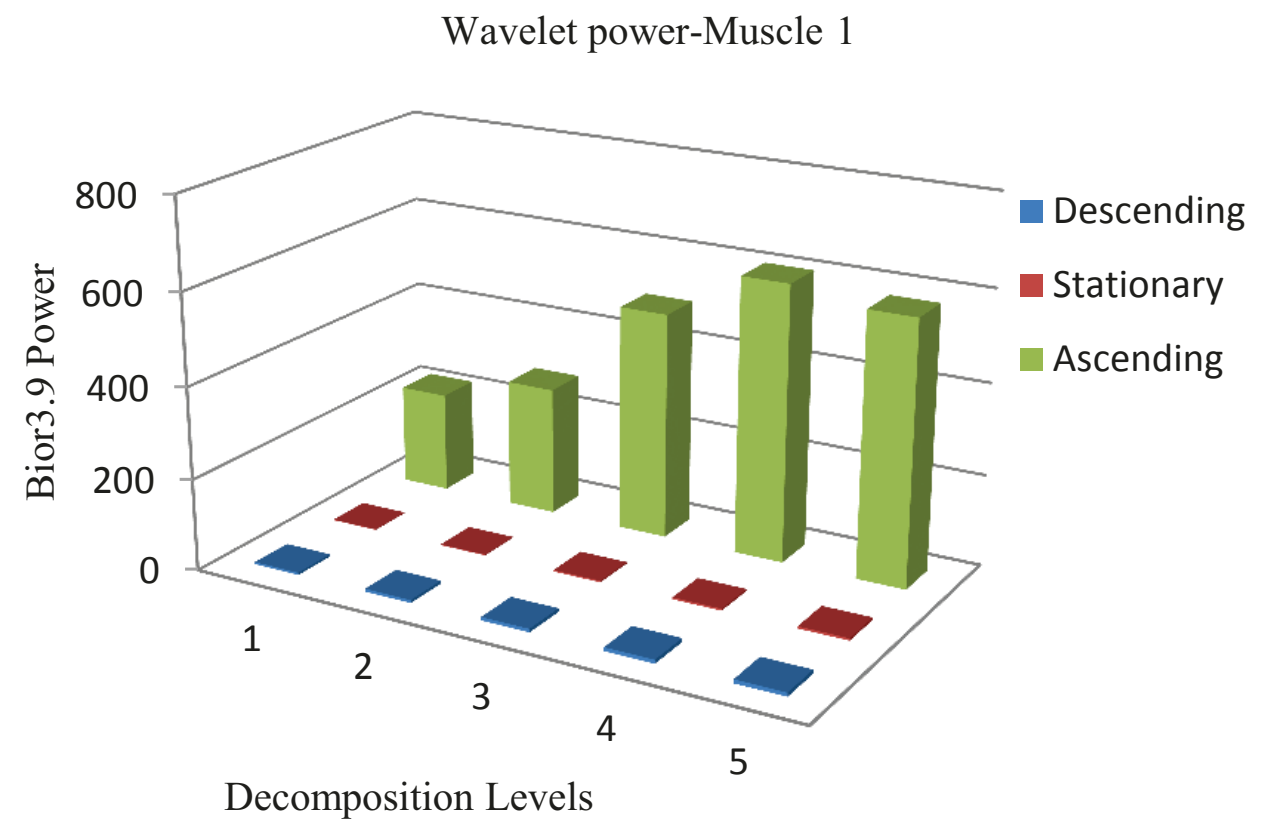

Figure 4.20: Example of Wavelet Power for Muscle 1 of One OR Subject 


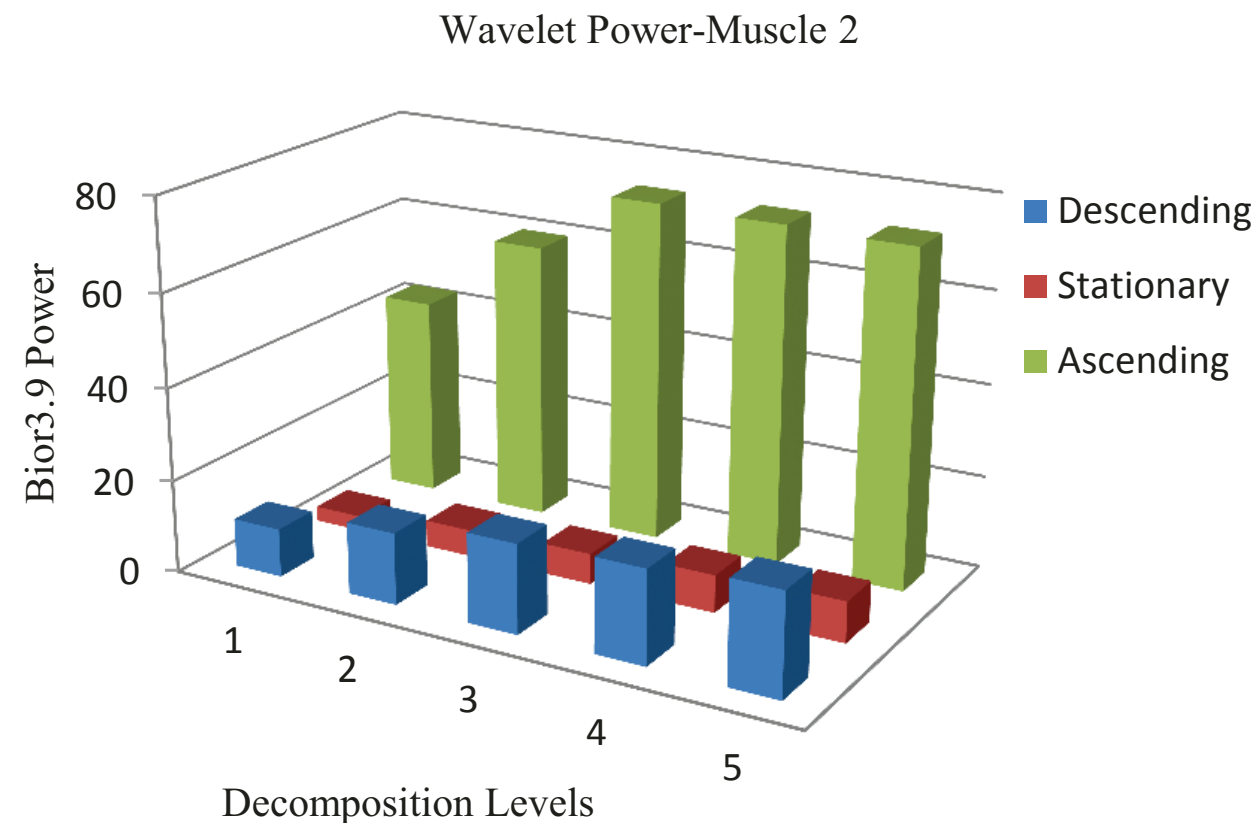

Figure 4.21: Example of Wavelet Power for Muscle 2 of One OR Subject 


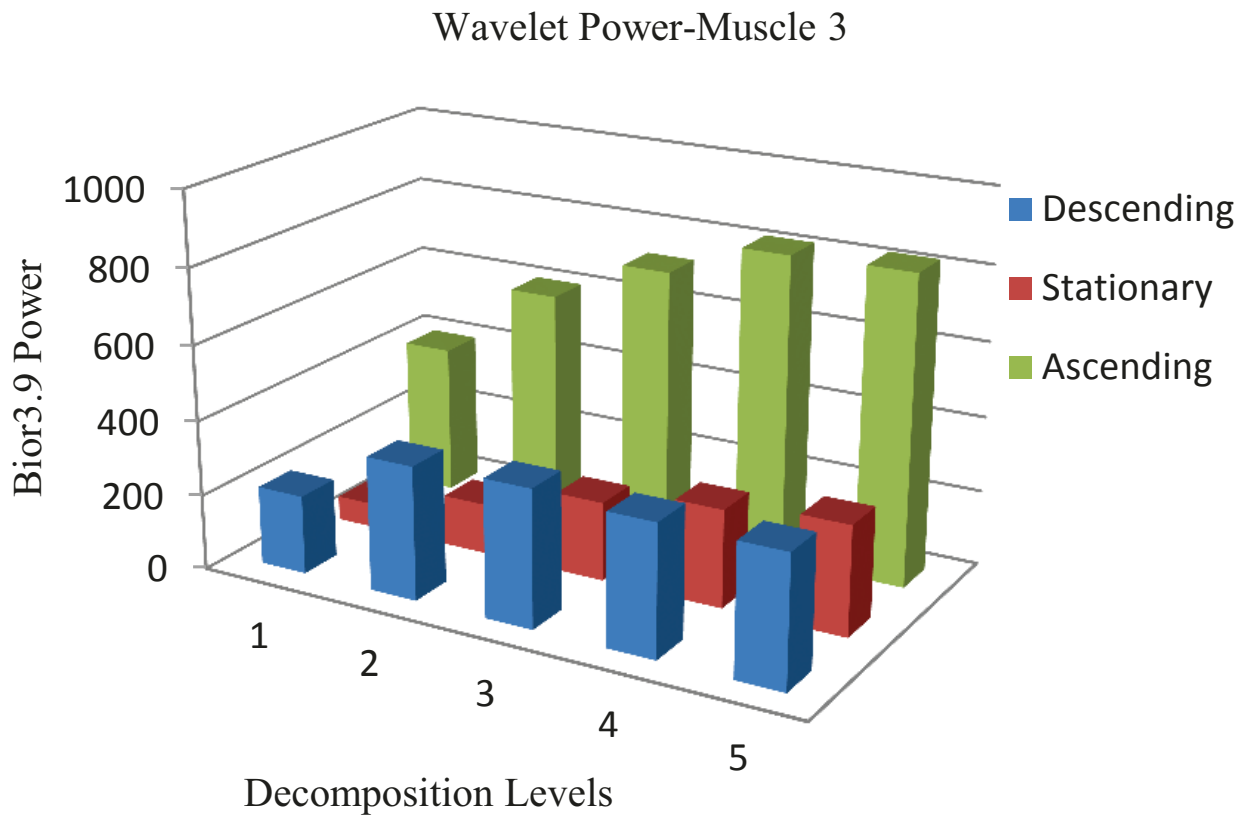

Figure 4.22: Example of Wavelet Power for Muscle 3 of One OR Subject

Additionally, the above scenario was repeated for all subjects from both groups. The subjects were categorized based on calculated power of each phase in Table 4.17. OR and CON groups were divided into three categories based on the squat phases (Table 4.17). All 16 subjects in the CON group used their muscle 1 in the ascending phase whereas, most of them used muscle 2 and muscle 3 in their descending phase. In OR group, muscle 1 was used in ascending the same as CON group. However, muscle 2 was used in ascending and muscle 3 in both descending and ascending phases. 
Table 4.17: Muscles used by CON and OR During Squat Cycle

\begin{tabular}{ccccc}
\hline & Descending & Stationary & Ascending & Total \\
\hline CON & & & & \\
\hline$M_{1}$ & 0 & 0 & 16 & 16 \\
$M_{2}$ & 10 & 2 & 4 & 16 \\
$M_{3}$ & 14 & 2 & 0 & 16 \\
\hline OR & & & & \\
\hline$M_{1}$ & 2 & 0 & 10 & 12 \\
$M_{2}$ & 4 & 0 & 8 & 12 \\
$M_{3}$ & 5 & 2 & 5 & 12 \\
\hline
\end{tabular}

Moreover, after calculating the power of the DWT coefficients at various levels, the power was then compared for the sEMG for OR and CON group of three muscles (GMax, TFL, RF). Figures 4.23, 4.24, 4.25 show the results of each muscle for both groups and Figures 4.26, 4.27 represent the same result but in a different orientation, this time we consider the power of each group separately. 
Muscle 1

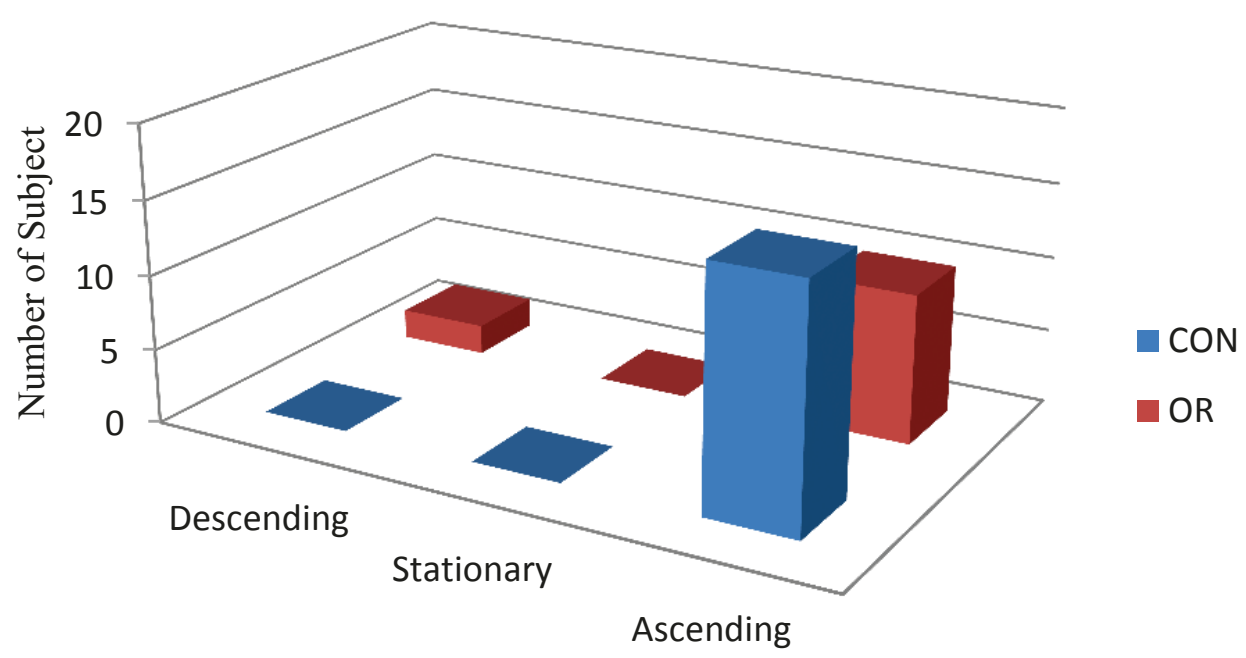

Squat Phases

Figure 4.23: Power of Muscle 1 for $\mathrm{CON}$ and OR during squat cycles 
Muscle 2

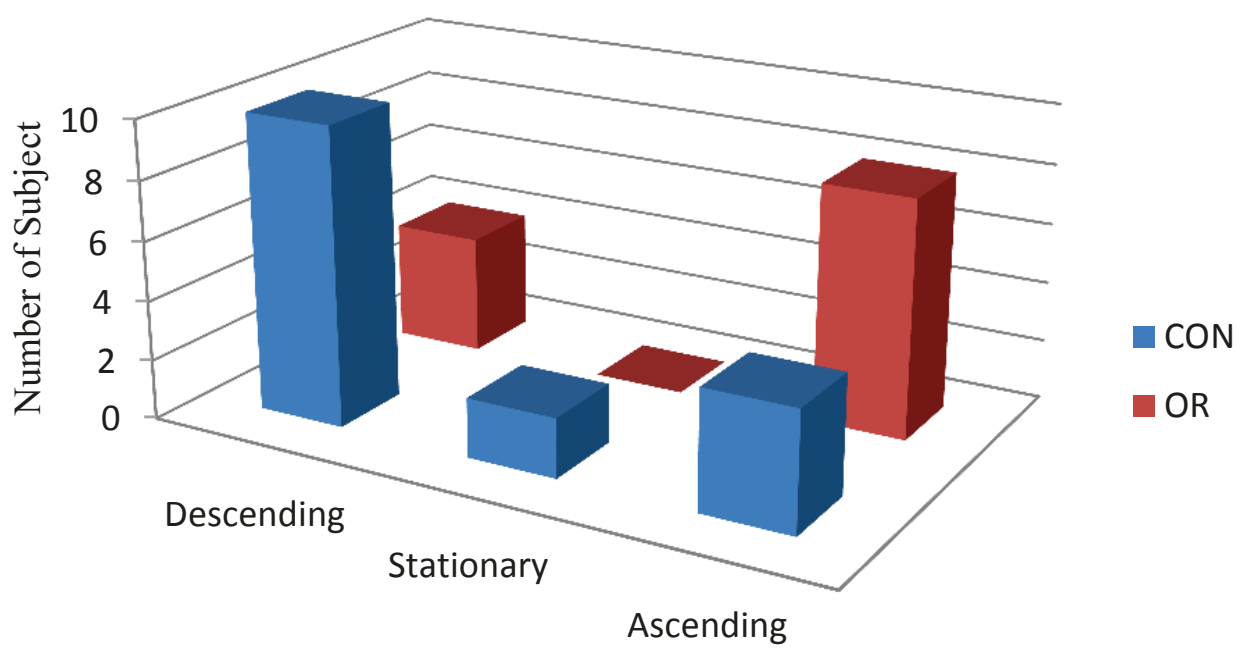

Squat Phases

Figure 4.24: Power of Muscle 2 for CON and OR during squat cycles 


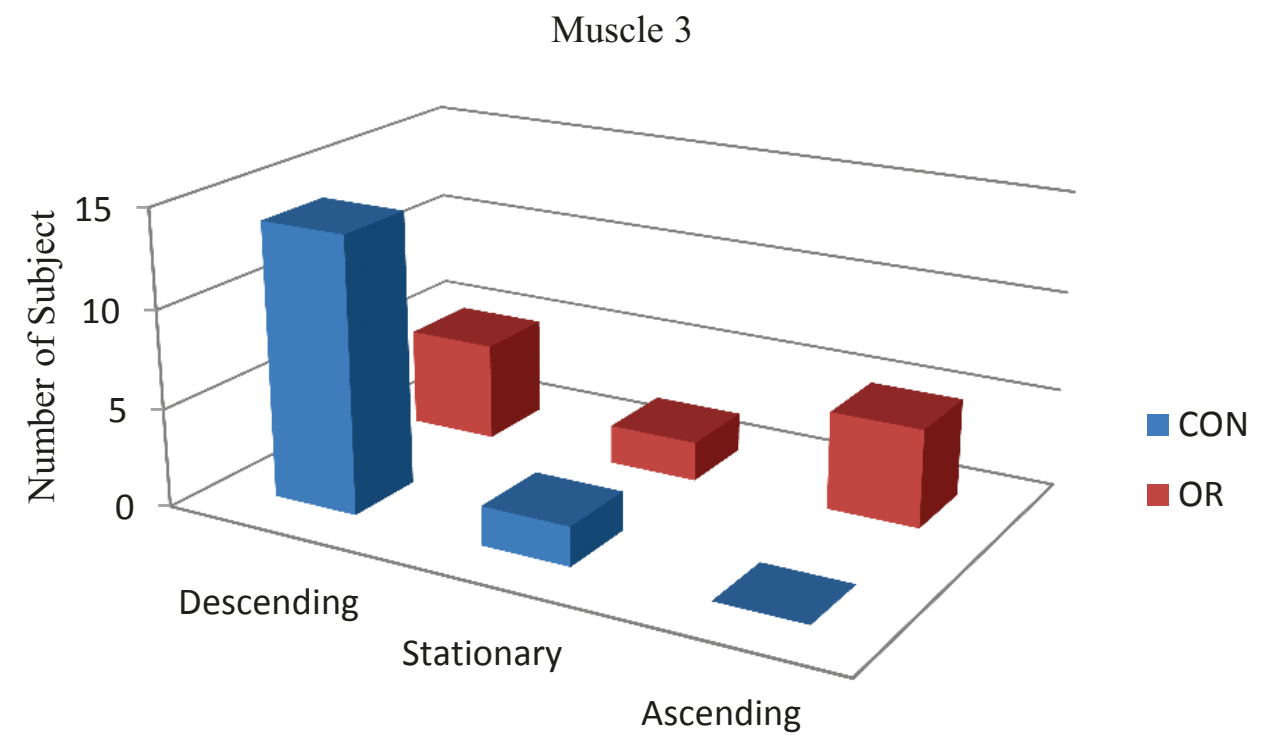

Squat Phases

Figure 4.25: Power of Muscle 3 for CON and OR during squat cycles 


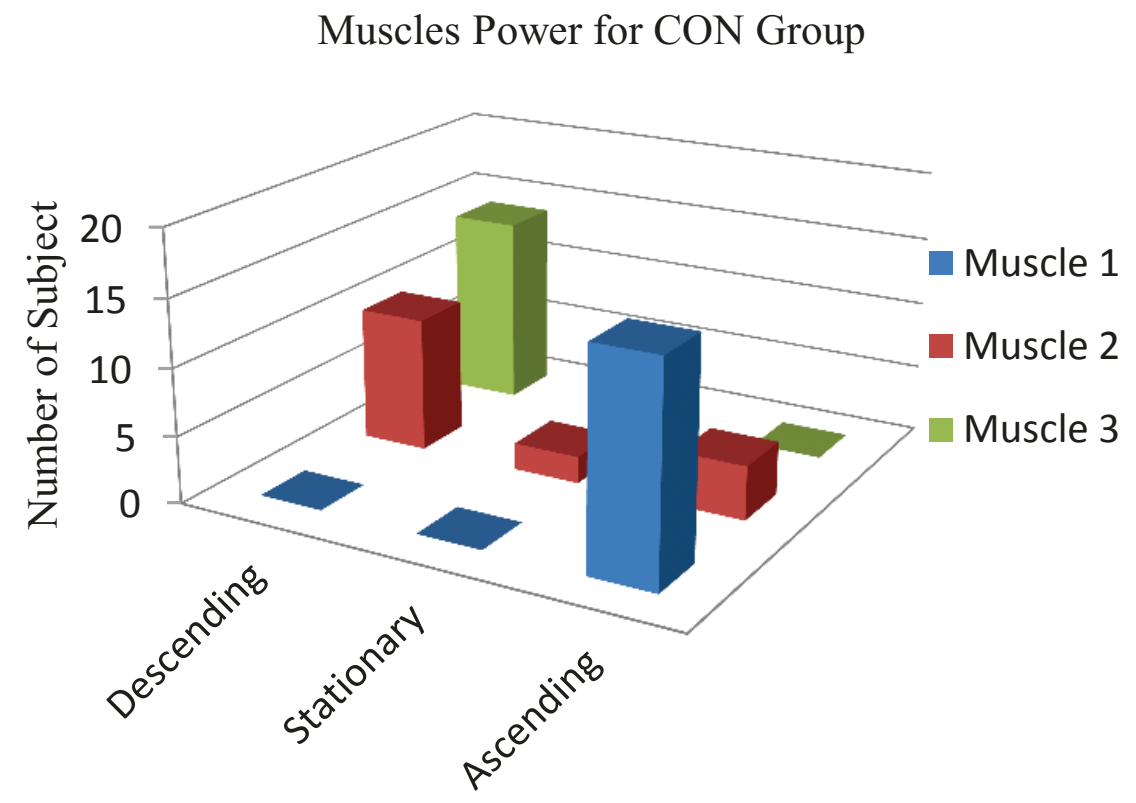

Squat Phases

Figure 4.26: Muscles Power for CON Group 


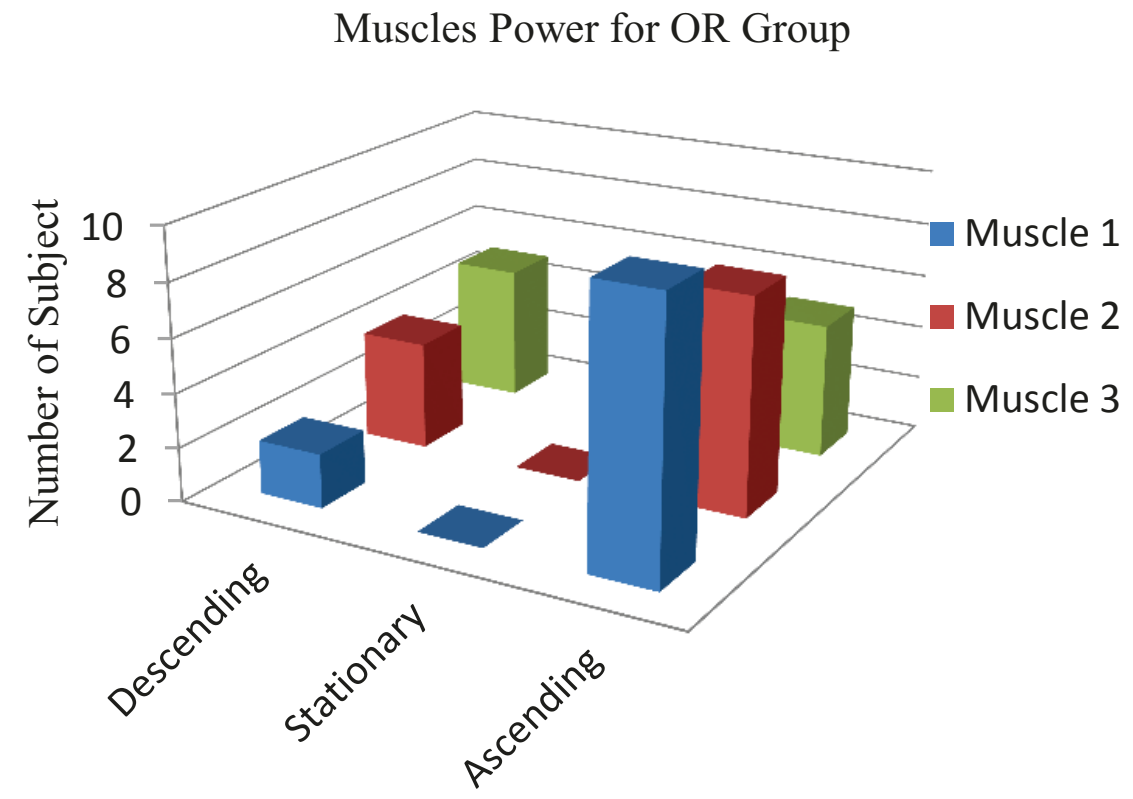

Squat Phases

Figure 4.27: Muscles Power for OR Group 
As indicated in above graphs, subjects from both groups used higher power in descending and ascending phases. In order to indicate whether there is a significant difference between these two groups in using their muscles during ascending (A) and descending (D) phases, the actual ratio A/D for CON and OR were computed. Therefore, we had a continuous number instead of a discrete number (the ratio) that gave more information on how much each muscle was more active during one phase compare to another phase. Tables 4.18 and 4.19 illustrate the ratio of ascending over descending phases for one CON and OR subject respectively (refer to appendix B for all other subjects).

\begin{tabular}{cccccc} 
Table 4.18: Ratio A/D Phase for One CON Subject \\
\cline { 4 - 5 } & Level 1 & Level 2 & Level 3 & Level 4 & Level 5 \\
\hline$M_{1}$ & 7.136 & 8.070 & 10.591 & 8.993 & 9.057 \\
$M_{2}$ & 0.107 & 0.098 & 0.093 & 0.106 & 0.097 \\
$M_{3}$ & 0.281 & 0.291 & 0.260 & 0.261 & 0.267 \\
\hline
\end{tabular}

Table 4.19: Ratio A/D Phase for One OR Subject

\begin{tabular}{cccccc}
\hline & Level 1 & Level 2 & Level 3 & Level 4 & Level 5 \\
\hline & & & & & \\
\hline$M_{1}$ & 45.068 & 45.246 & 64.179 & 74.109 & 66.716 \\
$M_{2}$ & 4.147 & 3.876 & 3.814 & 3.603 & 3.266 \\
$M_{3}$ & 1.911 & 1.698 & 1.971 & 2.329 & 2.354 \\
\end{tabular}


By calculating the ratio we are still capable of seeing which phase was the most active; however we are also able to see the extend of activity. Therefore, we calculated the ratio of $\mathrm{A} / \mathrm{D}$ for every subject in $\mathrm{CON}$ and $\mathrm{OR}$ groups and then the average was calculated. The average for each muscle is provided in Table 4.20 with the standard deviation for each group.

Table 4.20: Average Ratio With Standard Deviation Values for Each Muscle in CON and OR Groups

\begin{tabular}{lcc}
\hline & CON & OR \\
\hline Muscle 1 (Gmax) & $13.380 \pm 1.902$ & $11.319 \pm 1.921$ \\
Muscle 2 (TFL) & $1.330 \pm 0.150$ & $1.829 \pm 0.205$ \\
Muscle 3 (RF) & $0.613 \pm 0.090$ & $1.975 \pm 0.260$ \\
\hline
\end{tabular}

The muscles power is plotted in Figure 4.28 for CON and OR group. In CON group muscle 1 and 2 were active in ascending phase whereas muscle 3 was active in descending phase. Moreover, in OR group all three muscles were active in the ascending phase. 
Muscles Power for CON and OR

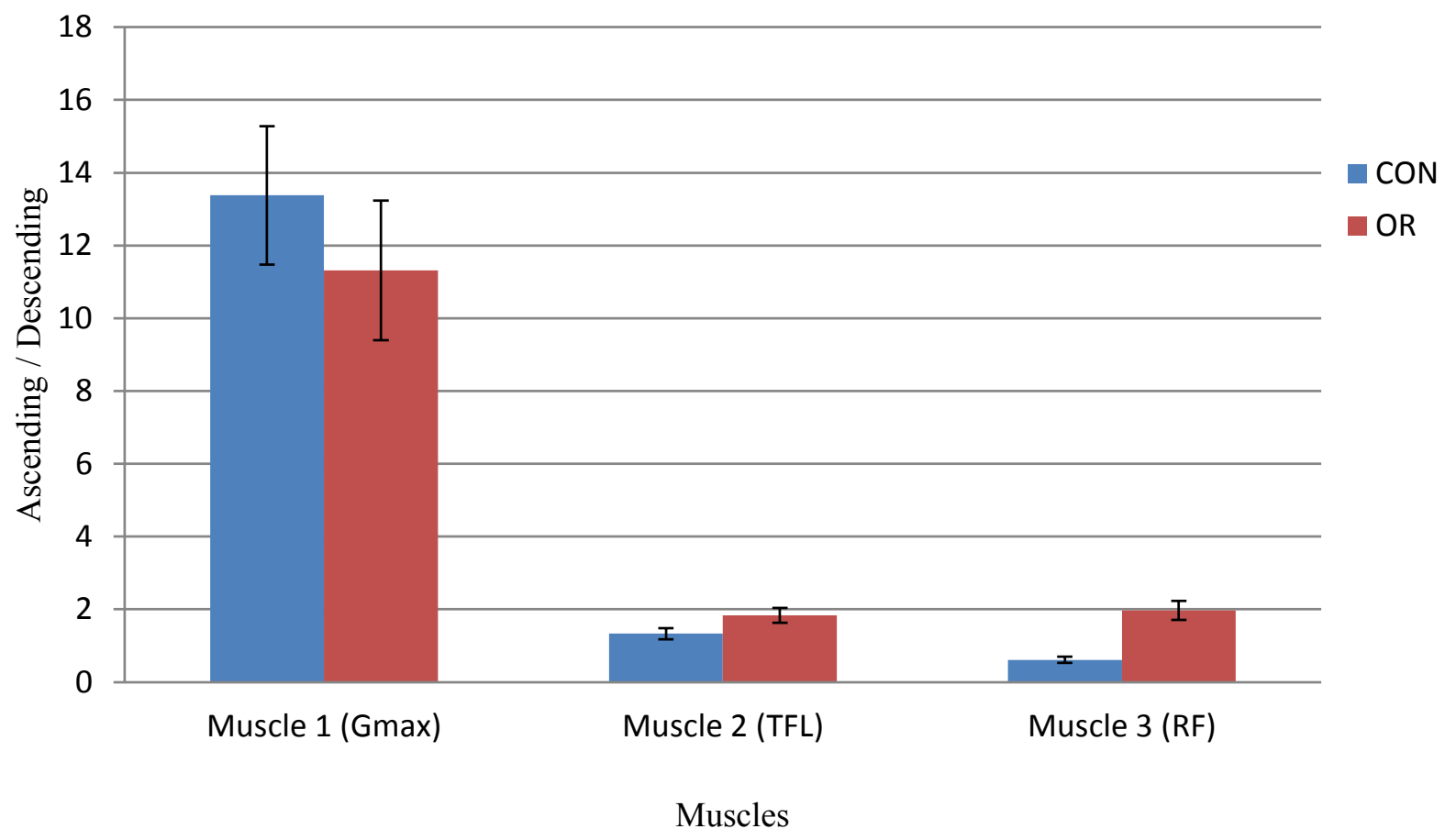

Figure 4.28: Average Ratio Muscle Power for CON and OR

\subsubsection{Algorithm}

In general, the described algorithm can be summarized as follow:

The raw EMG signals are collected from a participant for GMax, TFL, RF muscles during descending, stationary, and ascending phases of squat task. Then, the collected EMG signals are normalized. Bior3.9 wavelet function is applied to normalized EMG signals and the wavelet coefficients will computed. Based on wavelet coefficients the wavelet power spectrum is calculated. In addition, the ratio power which is the ratio of ascending power over descending power is computed in order to identify whether the subject has FAI or not. If the ratio value for RF muscle is less than zero, it means the participant used his/her RF muscle in descending phase and he/she belongs to 
CON group. Whereas, if the ratio value is greater than zero, it means he/she used his/her muscle in ascending phase and the subject belongs to FAI group.

\subsection{Statistical Analysis}

In this study an independent samples t-test was used to check if the two means (averages) from CON and OR groups are reliably different from one another. Each tvalue has a p-value that is the probability that the pattern of data in the sample could be produced by random data. The 2-tail t-test was applied to check the activities of muscles for CON and OR groups. Table 4.21 illustrates no significant difference was found for muscle 1 (GMax) and muscle 2 (TFL). Whereas muscle 3 (RF) almost shows a significant difference between CON and OR groups $(\mathrm{p}=0.0670)$ although the threshold $(\mathrm{p}=0.05)$ was not reached. This can be due to the fact that the sample size in this study was small and we require more samples for more reliable results.

Table 4.21: P-value for GMax, TFL, and RF Muscle

\begin{tabular}{lc}
\hline & P-value \\
\hline Muscle 1 (Gmax) & 0.7477 \\
Muscle 2 (TFL) & 0.4997 \\
Muscle 3 (RF) & 0.0670 \\
\hline
\end{tabular}

\subsection{Discussion}

Spectral properties of EMG signals have been defined by their power spectra. The shape of the power spectrum can be changed when the EMG signals are generated from different types of MU [72-74]. 
This study has indicated the wavelet transform method can be used to quantify features of the muscle activity for dynamic contraction. The fundamental properties of EMG spectra are conserved across dynamic contraction, therefore the WT will be a useful tool for studying EMG signals. The wavelet power spectrum of OR and CON groups was analyzed for GMax, TFL, and RF muscles in hip area by using Bior3.9 wavelet function in order to discriminate the two groups. The power was calculated for each muscle and an independent sample t-test was used to check the activity of three muscles in hip area to discriminate an OR patient from a healthy person. The p-value for GMax, TFL, and RF muscles is $0.7477,0.4997$, and 0.0670 respectively for 2-tail test. Therefore, RF muscle is almost statistically significant although the threshold ( $\mathrm{p}=0.05)$ was not reached due to small sample size. As a result, RF muscle is more active in ascending than descending phase for OR people, whereas it is more active in descending phase for CON people.

Muscle contraction is produced by a sequence of electrical and chemical events which start with an action potential which is created at the neuromuscular junction. Individual muscle fibers are classified into three primary muscle fiber types named type I, type IIA, and type IIB based on their contractile and metabolic properties. Type I is referred to slow twitch oxidative, type IIA is fast twitch oxidative and type IIB is fast twitch glycolytic [75]. These three types of muscle fibers have very different functional characteristics. Type I fiber is characterized by low force, power, speed production and high endurance. Type IIB has high force, power, speed production and low endurance, while type IIA indicates feature between the two other types. The MU consists of a single motoneuron and the group of muscle fiber it innervates. All muscle fibers in a single MU contain the same muscle fiber type. Three types of 
MUs (slow, fast fatigue-resistant, and fast fatigable) are categorized on the basis of their twitch speed and fatigability. The slow twitch MU is small and can produce less force compare to fast twitch MU. Type I muscle MUs contract slower, and they reach to peak power slower and highly resistant to fatigue compare to type II fast twitch MUs. Type IIA and IIB are capable of the same amount of peak force, however type IIA fibers take longer to reach their peak power compare to type IIB. Therefore, the total peak power by type IIB is higher than type IIA. In other words, type I has low intensity, lower frequency, and low power compare to type II.

The average ratio (ascending over descending phase) power for GMax, TFL, and RF muscles, as indicated in Table 4.20, presents the power of RF muscle is higher in OR group than CON group. The faster motor units generate higher frequencies in their power spectra [76]. Thus, the larger numbers of MUs are recruitment in RF muscle for OR than CON. Therefore, type II (fast twitch) MUs are active in OR group, while in CON group type I (slow twitch) MUs are used. 


\section{Chapter 5}

\section{Conclusions and Future Work}

The algorithm developed in this study aimed to automatically discriminate CON and OR groups based on sEMG recorded from three hip muscles (GMax, TFL, and RF) during dynamic contraction. The program was capable of identifying OR from healthy people by analyzing the activity of hip muscles.

The novel method developed in this study was used to analyze EMG signals recorded from GMax, TFL, and RF muscles for $16 \mathrm{CON}$ and 14 OR subjects during squat cycles. EMG signals are non-stationary signals, therefore time-frequency analysis based on wavelet transform methods were used. In this study the DWT was selected and various wavelet functions from this WT method were applied to EMG signals from three muscles in order to select the best possible energy localization in the time-frequency plane. This analysis showed the Bior3.9 wavelet function provided higher amount of energy for most of our subjects. By selecting Bior3.9 the wavelet power spectrum was computed for CON and OR during 5 levels of decomposition. The result indicated the RF muscle (muscle 3) is more active in ascending phase than 
descending phase for OR, while it is more active in descending phase for CON.

During dynamic contraction there is a progressive recruitment of faster MUs in OR group during ascending phase. EMG activity at higher frequencies correlated with higher contractile force, and with the progressively faster types of MU which can be assumed to be recruited. Thus, during dynamic contraction the higher wavelet power in RF muscle of OR group demonstrates that faster MUs were active, while lower power in CON group related to the fact that slower MUs were active.

This research work showed that the proposed algorithm is able to find a good solution for pre-screening problem. Nevertheless, some more improvements could be achieved. In this algorithm, only the three muscles of hip area were considered, this might be different if we consider all the muscles in the hip area. Another limitation is that the physical activity and age parameters of subjects were not available. Therefore, research should be conducted in a wider range, parameters like physical activity, age, and gender could be considered.

Some future work from this thesis may consist of considering a larger sample size for more accurate and reliable values. Ultimate goal can be developing a software application which can assist doctors and physicians to diagnose FAI faster and easier. 


\section{Appendix A}

In this appendix, we present numerical results for this study including all tables for different steps.

\section{IEMG and Time Normalized EMG}

The IEMG and time normalized values for CON and OR groups are presented in Table A.1, A.2, and A.3 for muscle 1, 2, and 3 respectively. 
Table A.1: IEMG and Time Normalized Values for Muscle 1 Without Outliers

\begin{tabular}{|c|c|c|c|}
\hline Subject & Integrated EMG & Length of Signal & Time Normalization \\
\hline \multicolumn{4}{|l|}{$\mathrm{CON}$} \\
\hline 1 & 377.219 & 5180 & 0.073 \\
\hline 2 & 478.673 & 4415 & 0.108 \\
\hline 5 & 298.995 & 4745 & 0.063 \\
\hline 6 & 452.587 & 8345 & 0.054 \\
\hline 7 & 360.346 & 3870 & 0.093 \\
\hline 10 & 459.621 & 6275 & 0.073 \\
\hline 11 & 379.996 & 5035 & 0.075 \\
\hline 12 & 447.927 & 5575 & 0.080 \\
\hline 15 & 168.690 & 4595 & 0.037 \\
\hline 17 & 255.802 & 4730 & 0.054 \\
\hline 19 & 1585.495 & 7500 & 0.211 \\
\hline 20 & 525.173 & 4070 & 0.129 \\
\hline 22 & 174.778 & 4640 & 0.038 \\
\hline 23 & 309.871 & 3050 & 0.102 \\
\hline 24 & 238.147 & 5065 & 0.047 \\
\hline 25 & 305.486 & 5150 & 0.060 \\
\hline \multicolumn{4}{|l|}{ OR } \\
\hline 3 & 251.270 & 4040 & 0.062 \\
\hline 8 & 470.763 & 6370 & 0.074 \\
\hline 9 & 221.722 & 6890 & 0.032 \\
\hline 13 & 521.575 & 4505 & 0.116 \\
\hline 14 & 592.230 & 10580 & 0.056 \\
\hline 16 & 474.705 & 6460 & 0.073 \\
\hline 18 & 418.132 & 6575 & 0.064 \\
\hline 21 & 531.754 & 7365 & 0.072 \\
\hline 27 & 2454.064 & 6100 & 0.402 \\
\hline 29 & 484.184 & 3365 & 0.144 \\
\hline
\end{tabular}


Table A.2: IEMG and Time Normalized Values for Muscle 2 Without Outliers

\begin{tabular}{|c|c|c|c|}
\hline Subject & Integrated EMG & Length of Signal & Time Normalization \\
\hline \multicolumn{4}{|l|}{$\mathrm{CON}$} \\
\hline 1 & 403.563 & 5180 & 0.078 \\
\hline 2 & 842.446 & 4415 & 0.191 \\
\hline 5 & 905.356 & 4745 & 0.191 \\
\hline 6 & 277.228 & 8345 & 0.033 \\
\hline 7 & 443.272 & 3870 & 0.115 \\
\hline 10 & 424.274 & 6275 & 0.068 \\
\hline 11 & 114.021 & 5035 & 0.023 \\
\hline 12 & 59.486 & 5575 & 0.011 \\
\hline 15 & 90.380 & 4595 & 0.020 \\
\hline 17 & 207.423 & 4730 & 0.044 \\
\hline 19 & 997.866 & 7500 & 0.133 \\
\hline 20 & 282.975 & 4070 & 0.070 \\
\hline 22 & 330.157 & 4640 & 0.071 \\
\hline 23 & 104.439 & 3050 & 0.034 \\
\hline 24 & 771.032 & 5065 & 0.152 \\
\hline 25 & 230.499 & 5150 & 0.045 \\
\hline \multicolumn{4}{|l|}{ OR } \\
\hline 3 & 141.236 & 4040 & 0.035 \\
\hline 8 & 1545.059 & 6370 & 0.243 \\
\hline 9 & 1142.234 & 6890 & 0.166 \\
\hline 13 & 185.388 & 4505 & 0.041 \\
\hline 14 & 202.193 & 10580 & 0.019 \\
\hline 16 & 230.616 & 6460 & 0.036 \\
\hline 18 & 371.761 & 6575 & 0.057 \\
\hline 21 & 399.293 & 7365 & 0.054 \\
\hline 27 & 654.696 & 6100 & 0.107 \\
\hline 28 & 163.525 & 3095 & 0.053 \\
\hline 29 & 286.109 & 3365 & 0.085 \\
\hline 30 & 531.612 & 4950 & 0.107 \\
\hline
\end{tabular}


Table A.3: IEMG and Time Normalized Values for Muscle 3 Without Outliers

\begin{tabular}{|c|c|c|c|}
\hline Subject & Integrated EMG & Length of Signal & Time Normalization \\
\hline \multicolumn{4}{|l|}{$\mathrm{CON}$} \\
\hline 1 & 641.209 & 5180 & 0.124 \\
\hline 2 & 343.034 & 4415 & 0.078 \\
\hline 5 & 698.830 & 4745 & 0.147 \\
\hline 6 & 1835.176 & 8345 & 0.220 \\
\hline 7 & 1225.814 & 3870 & 0.317 \\
\hline 10 & 980.394 & 6275 & 0.156 \\
\hline 11 & 680.335 & 5035 & 0.135 \\
\hline 12 & 538.071 & 5575 & 0.097 \\
\hline 15 & 554.491 & 4595 & 0.121 \\
\hline 17 & 948.499 & 4730 & 0.201 \\
\hline 19 & 1743.782 & 7500 & 0.233 \\
\hline 22 & 1007.532 & 4640 & 0.217 \\
\hline 23 & 467.060 & 3050 & 0.153 \\
\hline 24 & 549.610 & 5065 & 0.109 \\
\hline 25 & 1366.655 & 5150 & 0.265 \\
\hline \multicolumn{4}{|l|}{ OR } \\
\hline 3 & 848.363 & 4040 & 0.210 \\
\hline 8 & 1026.715 & 6370 & 0.161 \\
\hline 9 & 617.380 & 6890 & 0.090 \\
\hline 13 & 752.812 & 4505 & 0.167 \\
\hline 14 & 1588.370 & 10580 & 0.150 \\
\hline 16 & 657.576 & 6460 & 0.102 \\
\hline 18 & 1369.372 & 6575 & 0.208 \\
\hline 21 & 1246.705 & 7365 & 0.169 \\
\hline 27 & 135.664 & 6100 & 0.022 \\
\hline 28 & 85.839 & 3095 & 0.028 \\
\hline 29 & 787.682 & 3365 & 0.234 \\
\hline 30 & 288.251 & 4950 & 0.0583 \\
\hline
\end{tabular}




\section{Average Energy}

The highest amount of energy for each subject during squat cycles was collected in

Table A.4 (for muscle 1), A.5 (for muscle 2), and A.6 (for muscle 3).

Table A.4: Average Energy for Muscle 1 of CON and OR Group

\begin{tabular}{|c|c|c|c|c|}
\hline Subject & Descending & Stationary & Ascending & Average Energy \\
\hline \multicolumn{5}{|l|}{$\mathrm{CON}$} \\
\hline 1 & 34.821 & 28.937 & 47.165 & 36.974 \\
\hline 2 & 40.428 & 43.197 & 44.544 & 42.723 \\
\hline 5 & 42.700 & 36.855 & 48.691 & 42.749 \\
\hline 6 & 41.011 & 41.807 & 41.703 & 41.507 \\
\hline 7 & 41.180 & 34.774 & 59.151 & 45.035 \\
\hline 10 & 29.935 & 65.633 & 44.656 & 46.742 \\
\hline 11 & 34.709 & 18.791 & 51.679 & 35.060 \\
\hline 12 & 27.395 & 16.200 & 55.434 & 33.010 \\
\hline 15 & 35.523 & 35.940 & 52.381 & 41.281 \\
\hline 17 & 44.621 & 57.504 & 46.546 & 49.557 \\
\hline 19 & 41.394 & 40.440 & 42.369 & 41.401 \\
\hline 20 & 34.317 & 44.924 & 52.163 & 43.801 \\
\hline 22 & 40.844 & 31.784 & 44.238 & 38.956 \\
\hline 23 & 20.843 & 50.942 & 51.924 & 41.236 \\
\hline 24 & 24.111 & 30.651 & 36.600 & 30.454 \\
\hline 25 & 83.975 & 86.779 & 67.249 & 79.334 \\
\hline \multicolumn{5}{|l|}{ OR } \\
\hline 3 & 40.507 & 42.874 & 51.114 & 44.832 \\
\hline 8 & 33.274 & 45.506 & 40.249 & 39.676 \\
\hline 9 & 29.972 & 41.519 & 39.851 & 37.114 \\
\hline 13 & 29.960 & 35.027 & 50.869 & 38.619 \\
\hline 14 & 30.211 & 17.301 & 52.477 & 33.330 \\
\hline 16 & 57.215 & 23.221 & 42.312 & 40.916 \\
\hline 18 & 25.014 & 21.505 & 38.120 & 28.209 \\
\hline 21 & 23.002 & 49.830 & 45.561 & 39.464 \\
\hline 27 & 40.816 & 35.219 & 53.997 & 43.344 \\
\hline 28 & 51.969 & 25.079 & 35.288 & 37.445 \\
\hline 29 & 28.809 & 48.809 & 52.186 & 43.268 \\
\hline 30 & 40.138 & 64.222 & 42.989 & 49.116 \\
\hline
\end{tabular}


Table A.5: Average Energy for Muscle 2 of CON and OR Group

\begin{tabular}{|c|c|c|c|c|}
\hline Subject & Descending & Stationary & Ascending & Average Energy \\
\hline \multicolumn{5}{|l|}{$\mathrm{CON}$} \\
\hline 1 & 46.301 & 33.355 & 44.453 & 41.370 \\
\hline 2 & 44.891 & 30.897 & 49.625 & 41.804 \\
\hline 5 & 51.561 & 20.806 & 43.936 & 38.768 \\
\hline 6 & 58.466 & 46.951 & 48.699 & 51.372 \\
\hline 7 & 42.844 & 68.753 & 27.690 & 46.429 \\
\hline 10 & 47.135 & 41.739 & 34.892 & 41.256 \\
\hline 11 & 44.020 & 24.313 & 51.362 & 39.898 \\
\hline 12 & 34.282 & 34.522 & 37.990 & 35.598 \\
\hline 15 & 37.376 & 62.156 & 41.907 & 47.146 \\
\hline 17 & 37.008 & 53.272 & 40.148 & 43.476 \\
\hline 19 & 48.560 & 35.681 & 37.916 & 40.719 \\
\hline 20 & 29.409 & 31.572 & 45.075 & 35.352 \\
\hline 22 & 42.725 & 31.367 & 46.910 & 40.334 \\
\hline 23 & 27.970 & 63.559 & 48.762 & 46.763 \\
\hline 24 & 51.816 & 29.098 & 50.855 & 43.923 \\
\hline 25 & 46.676 & 51.640 & 41.822 & 46.713 \\
\hline \multicolumn{5}{|l|}{ OR } \\
\hline 3 & 65.335 & 14.629 & 63.650 & 47.871 \\
\hline 8 & 4.457 & 37.148 & 47.399 & 39.668 \\
\hline 9 & 53.844 & 69.241 & 57.732 & 60.272 \\
\hline 13 & 48.676 & 46.904 & 65.926 & 53.835 \\
\hline 14 & 44.611 & 68.225 & 37.629 & 50.155 \\
\hline 16 & 47.937 & 58.787 & 44.192 & 50.305 \\
\hline 18 & 39.019 & 21.140 & 34.374 & 31.511 \\
\hline 21 & 55.791 & 49.155 & 23.395 & 42.780 \\
\hline 27 & 38.200 & 62.166 & 33.639 & 44.668 \\
\hline 28 & 48.723 & 26.202 & 47.649 & 40.858 \\
\hline 29 & 31.079 & 42.749 & 37.554 & 37.127 \\
\hline 30 & 62.479 & 43.051 & 55.622 & 53.717 \\
\hline
\end{tabular}


Table A.6: Average Energy for Muscle 3 of CON and OR Group

\begin{tabular}{|c|c|c|c|c|}
\hline Subject & Descending & Stationary & Ascending & Average Energy \\
\hline \multicolumn{5}{|l|}{$\mathrm{CON}$} \\
\hline 1 & 33.417 & 33.897 & 48.612 & 38.642 \\
\hline 2 & 43.023 & 25.366 & 47.628 & 38.672 \\
\hline 5 & 39.288 & 38.864 & 55.221 & 44.458 \\
\hline 6 & 60.512 & 39.533 & 45.451 & 48.499 \\
\hline 7 & 39.974 & 28.444 & 53.880 & 40.766 \\
\hline 10 & 54.559 & 38.159 & 48.273 & 46.997 \\
\hline 11 & 44.214 & 53.758 & 44.176 & 47.383 \\
\hline 12 & 40.967 & 35.514 & 37.913 & 38.131 \\
\hline 15 & 39.586 & 44.222 & 46.045 & 43.284 \\
\hline 17 & 46.372 & 50.034 & 60.862 & 52.423 \\
\hline 19 & 47.197 & 39.787 & 38.417 & 41.800 \\
\hline 20 & 51.378 & 43.229 & 64.525 & 53.044 \\
\hline 22 & 45.996 & 26.917 & 54.128 & 42.347 \\
\hline 23 & 39.772 & 53.031 & 57.622 & 50.142 \\
\hline 24 & 54.910 & 34.084 & 55.928 & 48.307 \\
\hline 25 & 37.046 & 59.337 & 50.806 & 49.063 \\
\hline \multicolumn{5}{|l|}{ OR } \\
\hline 3 & 37.977 & 59.146 & 35.223 & 44.115 \\
\hline 8 & 41.644 & 29.465 & 42.929 & 38.013 \\
\hline 9 & 43.886 & 51.979 & 44.223 & 46.696 \\
\hline 13 & 52.162 & 40.540 & 40.289 & 44.331 \\
\hline 14 & 42.411 & 52.464 & 40.796 & 45.223 \\
\hline 16 & 56.530 & 47.099 & 45.627 & 49.752 \\
\hline 18 & 45.523 & 58.988 & 56.995 & 53.835 \\
\hline 21 & 43.136 & 66.346 & 41.533 & 50.338 \\
\hline 27 & 45.996 & 35.458 & 50.720 & 44.058 \\
\hline 28 & 40.034 & 45.528 & 47.400 & 44.321 \\
\hline 29 & 59.442 & 38.627 & 43.130 & 47.066 \\
\hline 30 & 81.126 & 76.039 & 52.082 & 69.749 \\
\hline
\end{tabular}




\section{Wavelet Power Spectrum}

\section{Muscle 1 - CON:}

Tables A.7- A.22 show the power values for muscle 1 of CON group after 5 decomposition levels.

Table A.7: Wavelet Power Spectrum Calculations for Muscle 1-CON Subjects 1

\begin{tabular}{cccccc}
\hline & Level 1 & Level 2 & Level 3 & Level 4 & Level 5 \\
\hline D & 15.234 & 21.183 & 25.326 & 27.768 & 28.379 \\
S & 4.848 & 7.634 & 12.941 & 17.722 & 18.016 \\
A & 58.217 & 78.200 & 130.362 & 157.882 & 154.783 \\
\hline
\end{tabular}

Table A.8: Wavelet Power Spectrum Calculations for Muscle 1-CON Subjects 2

\begin{tabular}{cccccc}
\hline & Level 1 & Level 2 & Level 3 & Level 4 & Level 5 \\
\hline $\mathrm{D}$ & 5.887 & 7.406 & 9.834 & 12.364 & 12.442 \\
$\mathrm{~S}$ & 0.669 & 1.131 & 1.449 & 1.899 & 2.529 \\
$\mathrm{~A}$ & 42.010 & 59.763 & 104.155 & 111.192 & 112.685 \\
\hline
\end{tabular}

Table A.9: Wavelet Power Spectrum Calculations for Muscle 1-CON Subjects 3

\begin{tabular}{cccccc}
\hline & Level 1 & Level 2 & Level 3 & Level 4 & Level 5 \\
\hline $\mathrm{D}$ & 1.705 & 2.287 & 3.041 & 3.271 & 3.485 \\
$\mathrm{~S}$ & 0.178 & 0.398 & 0.5738 & 0.754 & 0.843 \\
$\mathrm{~A}$ & 16.512 & 24.076 & 37.216 & 32.518 & 32.220 \\
\hline
\end{tabular}

Table A.10: Wavelet Power Spectrum Calculations for Muscle 1-CON Subjects 4

\begin{tabular}{cccccc}
\hline & Level 1 & Level 2 & Level 3 & Level 4 & Level 5 \\
\hline $\mathrm{D}$ & 2.717 & 3.585 & 4.997 & 5.605 & 5.507 \\
$\mathrm{~S}$ & 3.257 & 4.778 & 6.139 & 6.962 & 7.358 \\
$\mathrm{~A}$ & 81.265 & 115.329 & 172.295 & 229.732 & 224.537 \\
\hline
\end{tabular}


Table A.11: Wavelet Power Spectrum Calculations for Muscle 1-CON Subjects 5

\begin{tabular}{cccccc}
\hline & Level 1 & Level 2 & Level 3 & Level 4 & Level 5 \\
\hline $\mathrm{D}$ & 9.840 & 14.117 & 19.819 & 20.992 & 20.308 \\
$\mathrm{~S}$ & 1.154 & 2.263 & 3.452 & 4.022 & 4.285 \\
$\mathrm{~A}$ & 147.440 & 192.286 & 315.352 & 281.735 & 263.630
\end{tabular}

Table A.12: Wavelet Power Spectrum Calculations for Muscle 1-CON Subjects 6

\begin{tabular}{cccccc}
\hline & Level 1 & Level 2 & Level 3 & Level 4 & Level 5 \\
\hline $\mathrm{D}$ & 6.342 & 8.403 & 12.645 & 13.330 & 13.906 \\
$\mathrm{~S}$ & 0.660 & 0.934 & 1.617 & 2.027 & 2.347 \\
$\mathrm{~A}$ & 45.485 & 57.485 & 103.298 & 104.419 & 94.439 \\
\hline
\end{tabular}

Table A.13: Wavelet Power Spectrum Calculations for Muscle 1-CON Subjects 7

\begin{tabular}{cccccc}
\hline & Level 1 & Level 2 & Level 3 & Level 4 & Level 5 \\
\hline $\mathrm{D}$ & 13.331 & 18.071 & 27.209 & 30.189 & 29.212 \\
$\mathrm{~S}$ & 2.211 & 3.260 & 5.651 & 8.687 & 9.246 \\
$\mathrm{~A}$ & 32.728 & 42.676 & 68.701 & 79.405 & 73.886 \\
\hline
\end{tabular}

Table A.14: Wavelet Power Spectrum Calculations for Muscle 1-CON Subjects 8

\begin{tabular}{cccccc}
\hline & Level 1 & Level 2 & Level 3 & Level 4 & Level 5 \\
\hline $\mathrm{D}$ & 6.338 & 8.009 & 12.342 & 11.949 & 12.260 \\
$\mathrm{~S}$ & 1.115 & 1.8246 & 2.657 & 3.217 & 3.753 \\
$\mathrm{~A}$ & 69.142 & 88.967 & 147.776 & 192.049 & 178.097 \\
\hline
\end{tabular}

Table A.15: Wavelet Power Spectrum Calculations for Muscle 1-CON Subjects 9

\begin{tabular}{cccccc}
\hline & Level 1 & Level 2 & Level 3 & Level 4 & Level 5 \\
\hline D & 13.698 & 18.6612 & 24.958 & 29.426 & 31.986 \\
S & 3.834 & 5.7188 & 7.303 & 9.745 & 14.457 \\
A & 84.277 & 120.986 & 182.845 & 166.209 & 160.437 \\
\hline
\end{tabular}


Table A.16: Wavelet Power Spectrum Calculations for Muscle 1-CON Subjects 10

\begin{tabular}{cccccc}
\hline & Level 1 & Level 2 & Level 3 & Level 4 & Level 5 \\
\hline $\mathrm{D}$ & 5.859 & 9.586 & 13.084 & 12.921 & 12.886 \\
$\mathrm{~S}$ & 0.507 & 0.754 & 1.286 & 1.593 & 1.642 \\
$\mathrm{~A}$ & 42.138 & 57.165 & 95.210 & 114.783 & 110.769
\end{tabular}

Table A.17: Wavelet Power Spectrum Calculations for Muscle 1-CON Subjects 11

\begin{tabular}{cccccc}
\hline & Level 1 & Level 2 & Level 3 & Level 4 & Level 5 \\
\hline D & 139.459 & 115.851 & 159.940 & 169.901 & 172.494 \\
S & 61.049 & 55.492 & 75.757 & 78.979 & 79.9168 \\
A & 593.848 & 803.997 & 1222.185 & 1464.309 & 1383.084 \\
\hline
\end{tabular}

Table A.18: Wavelet Power Spectrum Calculations for Muscle 1-CON Subjects 12

\begin{tabular}{cccccc}
\hline & Level 1 & Level 2 & Level 3 & Level 4 & Level 5 \\
\hline $\mathrm{D}$ & 8.143 & 10.336 & 14.402 & 16.052 & 15.823 \\
$\mathrm{~S}$ & 0.625 & 0.816 & 1.292 & 1.566 & 1.675 \\
$\mathrm{~A}$ & 166.033 & 249.799 & 379.322 & 381.062 & 374.548
\end{tabular}

Table A.19: Wavelet Power Spectrum Calculations for Muscle 1-CON Subjects 13

\begin{tabular}{cccccc}
\hline & Level 1 & Level 2 & Level 3 & Level 4 & Level 5 \\
\hline $\mathrm{D}$ & 4.006 & 5.690 & 8.551 & 9.019 & 9.386 \\
$\mathrm{~S}$ & 0.429 & 0.743 & 1.341 & 1.527 & 2.116 \\
$\mathrm{~A}$ & 9.098 & 12.177 & 23.103 & 32.680 & 33.683 \\
\hline
\end{tabular}

Table A.20: Wavelet Power Spectrum Calculations for Muscle 1-CON Subjects 14

\begin{tabular}{cccccc}
\hline & Level 1 & Level 2 & Level 3 & Level 4 & Level 5 \\
\hline $\mathrm{D}$ & 2.367 & 2.881 & 3.739 & 4.284 & 5.080 \\
$\mathrm{~S}$ & 2.436 & 5.996 & 17.040 & 25.856 & 31.524 \\
$\mathrm{~A}$ & 131.616 & 208.267 & 305.145 & 294.823 & 288.415 \\
\hline
\end{tabular}


Table A.21: Wavelet Power Spectrum Calculations for Muscle 1-CON Subjects 15

\begin{tabular}{cccccc}
\hline & Level 1 & Level 2 & Level 3 & Level 4 & Level 5 \\
\hline D & 6.169 & 8.179 & 10.085 & 10.465 & 11.390 \\
S & 0.404 & 0.617 & 0.840 & 1.097 & 1.350 \\
A & 12.270 & 15.614 & 21.767 & 21.735 & 20.899 \\
\hline
\end{tabular}

Table A.22: Wavelet Power Spectrum Calculations for Muscle 1-CON Subjects 16

\begin{tabular}{cccccc}
\hline & Level 1 & Level 2 & Level 3 & Level 4 & Level 5 \\
\hline $\mathrm{D}$ & 10.466 & 14.529 & 32.184 & 34.474 & 34.697 \\
$\mathrm{~S}$ & 1.235 & 1.938 & 4.913 & 6.624 & 6.745 \\
$\mathrm{~A}$ & 14.703 & 20.507 & 36.173 & 39.469 & 39.015 \\
\hline
\end{tabular}




\section{Muscle 1 - OR:}

Tables A.23- A.34 illustrate the power values for muscle 1 of OR group.

Table A.23: Wavelet Power Spectrum Calculations for Muscle 1-OR Subjects 1

\begin{tabular}{cccccc}
\hline & Level 1 & Level 2 & Level 3 & Level 4 & Level 5 \\
\hline $\mathrm{D}$ & 15.873 & 22.844 & 26.115 & 28.629 & 29.046 \\
$\mathrm{~S}$ & 0.676 & 1.149 & 1.852 & 2.6107 & 3.953 \\
$\mathrm{~A}$ & 33.964 & 47.320 & 75.404 & 74.402 & 73.645 \\
\hline
\end{tabular}

Table A.24: Wavelet Power Spectrum Calculations for Muscle 1-OR Subjects 2

\begin{tabular}{cccccc}
\hline & Level 1 & Level 2 & Level 3 & Level 4 & Level 5 \\
\hline $\mathrm{D}$ & 4.897 & 6.234 & 7.728 & 8.162 & 8.667 \\
$\mathrm{~S}$ & 1.083 & 1.525 & 3.229 & 4.137 & 4.935 \\
$\mathrm{~A}$ & 220.683 & 282.050 & 495.946 & 604.871 & 578.224 \\
\hline
\end{tabular}

Table A.25: Wavelet Power Spectrum Calculations for Muscle 1-OR Subjects 3

\begin{tabular}{cccccc}
\hline & Level 1 & Level 2 & Level 3 & Level 4 & Level 5 \\
\hline D & 968.466 & 1352.904 & 2125.791 & 2443.927 & 2378.981 \\
S & 403.874 & 647.025 & 1112.626 & 1728.159 & 2009.300 \\
A & 935.557 & 1426.209 & 2206.506 & 2312.612 & 2271.503 \\
\hline
\end{tabular}

Table A.26: Wavelet Power Spectrum Calculations for Muscle 1-OR Subjects 4

\begin{tabular}{cccccc}
\hline & Level 1 & Level 2 & Level 3 & Level 4 & Level 5 \\
\hline $\mathrm{D}$ & 7.544 & 9.566 & 11.586 & 12.037 & 12.321 \\
$\mathrm{~S}$ & 0.538 & 0.877 & 1.182 & 1.969 & 2.769 \\
$\mathrm{~A}$ & 34.007 & 45.324 & 77.463 & 88.965 & 91.871 \\
\hline
\end{tabular}


Table A.27: Wavelet Power Spectrum Calculations for Muscle 1-OR Subjects 5

\begin{tabular}{cccccc}
\hline & Level 1 & Level 2 & Level 3 & Level 4 & Level 5 \\
\hline $\mathrm{D}$ & 4.204 & 6.133 & 8.652 & 8.171 & 8.081 \\
$\mathrm{~S}$ & 0.552 & 1.011 & 1.232 & 1.266 & 1.389 \\
$\mathrm{~A}$ & 32.205 & 43.242 & 77.507 & 101.661 & 98.930 \\
\hline
\end{tabular}

Table A.28: Wavelet Power Spectrum Calculations for Muscle 1-OR Subjects 6

\begin{tabular}{cccccc}
\hline & Level 1 & Level 2 & Level 3 & Level 4 & Level 5 \\
\hline D & 11.203 & 16.052 & 22.063 & 23.174 & 23.617 \\
S & 0.372 & 0.617 & 1.020 & 1.420 & 1.435 \\
$\mathrm{~A}$ & 53.215 & 75.828 & 116.701 & 119.300 & 122.110
\end{tabular}

Table A.29: Wavelet Power Spectrum Calculations for Muscle 1-OR Subjects 7

\begin{tabular}{cccccc}
\hline & Level 1 & Level 2 & Level 3 & Level 4 & Level 5 \\
\hline $\mathrm{D}$ & 0.904 & 1.189 & 1.646 & 1.863 & 1.888 \\
$\mathrm{~S}$ & 0.124 & 0.216 & 0.396 & 0.559 & 0.693 \\
$\mathrm{~A}$ & 19.125 & 29.149 & 40.867 & 49.071 & 48.911 \\
\hline
\end{tabular}

Table A.30: Wavelet Power Spectrum Calculations for Muscle 1-OR Subjects 8

\begin{tabular}{cccccc}
\hline & Level 1 & Level 2 & Level 3 & Level 4 & Level 5 \\
\hline $\mathrm{D}$ & 11.881 & 17.341 & 21.973 & 24.537 & 25.443 \\
$\mathrm{~S}$ & 1.3618 & 2.155 & 2.250 & 2.334 & 2.845 \\
$\mathrm{~A}$ & 141.249 & 195.605 & 318.413 & 297.099 & 295.917 \\
\hline
\end{tabular}


Table A.31: Wavelet Power Spectrum Calculations for Muscle 1-OR Subjects 9

\begin{tabular}{cccccc}
\hline & Level 1 & Level 2 & Level 3 & Level 4 & Level 5 \\
\hline $\mathrm{D}$ & 25.499 & 39.360 & 57.141 & 55.785 & 57.272 \\
$\mathrm{~S}$ & 1.0288 & 1.531 & 1.937 & 2.417 & 2.885 \\
$\mathrm{~A}$ & 64.196 & 104.794 & 146.255 & 142.901 & 155.987 \\
\hline
\end{tabular}

Table A.32: Wavelet Power Spectrum Calculations for Muscle 1-OR Subjects 10

\begin{tabular}{cccccc}
\hline & Level 1 & Level 2 & Level 3 & Level 4 & Level 5 \\
\hline $\mathrm{D}$ & 15.106 & 19.112 & 22.409 & 24.782 & 25.494 \\
$\mathrm{~S}$ & 1.380 & 1.976 & 3.609 & 4.476 & 5.016 \\
$\mathrm{~A}$ & 73.372 & 98.384 & 165.753 & 177.427 & 169.235 \\
\hline
\end{tabular}

Table A.33: Wavelet Power Spectrum Calculations for Muscle 1-OR Subjects 11

\begin{tabular}{cccccc}
\hline & Level 1 & Level 2 & Level 3 & Level 4 & Level 5 \\
\hline D & 215.074 & 296.882 & 474.623 & 427.348 & 417.509 \\
S & 208.844 & 362.480 & 380.454 & 449.010 & 581.087 \\
A & 1189.976 & 2086.568 & 2735.580 & 2762.507 & 2778.652 \\
\hline
\end{tabular}

Table A.34: Wavelet Power Spectrum Calculations for Muscle 1-OR Subjects 12

\begin{tabular}{cccccc}
\hline & Level 1 & Level 2 & Level 3 & Level 4 & Level 5 \\
\hline D & 20423.076 & 30702.608 & 44432.748 & 40900.063 & 45473.032 \\
S & 2053.197 & 3491.464 & 6767.1330 & 10099.479 & 10841.255 \\
A & 11135.299 & 17183.720 & 22277.281 & 21759.664 & 21338.019 \\
\hline
\end{tabular}




\section{Muscle 2 - CON:}

Tables A.35 - A.50 indicate the power values for muscle 2 of CON group.

Table A.35: Wavelet Power Spectrum Calculations for Muscle 2-CON Subjects 1

\begin{tabular}{cccccc}
\hline & Level 1 & Level 2 & Level 3 & Level 4 & Level 5 \\
\hline $\mathrm{D}$ & 77.188 & 119.487 & 164.128 & 153.089 & 150.122 \\
$\mathrm{~S}$ & 4.224 & 6.686 & 11.103 & 12.400 & 14.202 \\
$\mathrm{~A}$ & 16.189 & 25.187 & 36.740 & 42.413 & 45.409 \\
\hline
\end{tabular}

Table A.36: Wavelet Power Spectrum Calculations for Muscle 2-CON Subjects 2

\begin{tabular}{cccccc}
\hline & Level 1 & Level 2 & Level 3 & Level 4 & Level 5 \\
\hline $\mathrm{D}$ & 20.990 & 26.399 & 35.836 & 46.449 & 39.715 \\
$\mathrm{~S}$ & 0.025 & 0.043 & 0.058 & 0.078 & 0.104 \\
$\mathrm{~A}$ & 2.244 & 2.593 & 3.320 & 4.911 & 3.860 \\
\hline
\end{tabular}

Table A.37: Wavelet Power Spectrum Calculations for Muscle 2-CON Subjects 3

\begin{tabular}{cccccc}
\hline & Level 1 & Level 2 & Level 3 & Level 4 & Level 5 \\
\hline $\mathrm{D}$ & 3.009 & 5.015 & 6.416 & 6.643 & 7.284 \\
$\mathrm{~S}$ & 0.806 & 1.274 & 2.413 & 3.575 & 4.085 \\
$\mathrm{~A}$ & 1.185 & 1.822 & 2.381 & 2.287 & 2.288 \\
\hline
\end{tabular}

Table A.38: Wavelet Power Spectrum Calculations for Muscle 2-CON Subjects 4

\begin{tabular}{cccccc}
\hline & Level 1 & Level 2 & Level 3 & Level 4 & Level 5 \\
\hline D & 22.319 & 31.656 & 46.558 & 55.991 & 52.927 \\
S & 52.990 & 82.217 & 117.911 & 144.729 & 158.481 \\
A & 11.905 & 16.976 & 32.807 & 47.837 & 50.425 \\
\hline
\end{tabular}


Table A.39: Wavelet Power Spectrum Calculations for Muscle 2-CON Subjects 5

\begin{tabular}{cccccc}
\hline & Level 1 & Level 2 & Level 3 & Level 4 & Level 5 \\
\hline $\mathrm{D}$ & 49.357 & 71.623 & 114.343 & 128.904 & 121.670 \\
$\mathrm{~S}$ & 3.532 & 5.519 & 7.761 & 9.022 & 9.828 \\
$\mathrm{~A}$ & 267.821 & 344.736 & 567.003 & 638.628 & 564.952 \\
\hline
\end{tabular}

Table A.40: Wavelet Power Spectrum Calculations for Muscle 2-CON Subjects 6

\begin{tabular}{cccccc}
\hline & Level 1 & Level 2 & Level 3 & Level 4 & Level 5 \\
\hline D & 477.181 & 668.557 & 1033.859 & 1515.541 & 1417.036 \\
S & 36.372 & 72.027 & 132.552 & 177.622 & 237.104 \\
A & 186.516 & 241.746 & 378.406 & 458.924 & 438.743 \\
\hline
\end{tabular}

Table A.41: Wavelet Power Spectrum Calculations for Muscle 2-CON Subjects 7

\begin{tabular}{cccccc}
\hline & Level 1 & Level 2 & Level 3 & Level 4 & Level 5 \\
\hline $\mathrm{D}$ & 6.103 & 9.154 & 15.058 & 15.201 & 14.769 \\
$\mathrm{~S}$ & 0.781 & 1.245 & 1.951 & 2.276 & 2.374 \\
$\mathrm{~A}$ & 10.135 & 15.562 & 21.699 & 21.514 & 20.754 \\
\hline
\end{tabular}

Table A.42: Wavelet Power Spectrum Calculations for Muscle 2-CON Subjects 8

\begin{tabular}{cccccc}
\hline & Level 1 & Level 2 & Level 3 & Level 4 & Level 5 \\
\hline D & 42.110 & 57.979 & 91.999 & 88.652 & 83.984 \\
S & 25.578 & 42.268 & 115.894 & 187.959 & 192.714 \\
A & 59.307 & 87.130 & 159.763 & 207.698 & 288.991 \\
\hline
\end{tabular}

Table A.43: Wavelet Power Spectrum Calculations for Muscle 2-CON Subjects 9

\begin{tabular}{cccccc}
\hline & Level 1 & Level 2 & Level 3 & Level 4 & Level 5 \\
\hline $\mathrm{D}$ & 0.680 & 1.009 & 1.314 & 1.388 & 1.397 \\
$\mathrm{~S}$ & 0.249 & 0.347 & 0.425 & 0.460 & 0.480 \\
$\mathrm{~A}$ & 1.132 & 1.583 & 2.285 & 2.330 & 2.342 \\
& & & & & \\
\hline
\end{tabular}


Table A.44: Wavelet Power Spectrum Calculations for Muscle 2-CON Subjects 10

\begin{tabular}{cccccc}
\hline & Level 1 & Level 2 & Level 3 & Level 4 & Level 5 \\
\hline $\mathrm{D}$ & 13.342 & 21.183 & 26.091 & 25.816 & 25.561 \\
$\mathrm{~S}$ & 1.134 & 2.591 & 3.384 & 3.823 & 3.864 \\
$\mathrm{~A}$ & 11.156 & 15.723 & 24.029 & 25.276 & 26.616 \\
\hline
\end{tabular}

Table A.45: Wavelet Power Spectrum Calculations for Muscle 2-CON Subjects 11

\begin{tabular}{cccccc}
\hline & Level 1 & Level 2 & Level 3 & Level 4 & Level 5 \\
\hline D & 138.710 & 202.048 & 285.997 & 295.704 & 293.425 \\
S & 183.614 & 267.888 & 306.420 & 323.075 & 317.885 \\
A & 92.518 & 126.435 & 192.791 & 230.787 & 236.527 \\
\hline
\end{tabular}

Table A.46: Wavelet Power Spectrum Calculations for Muscle 2-CON Subjects 12

\begin{tabular}{cccccc}
\hline & Level 1 & Level 2 & Level 3 & Level 4 & Level 5 \\
\hline $\mathrm{D}$ & 27.124 & 34.187 & 50.642 & 56.277 & 60.207 \\
$\mathrm{~S}$ & 1.849 & 2.975 & 4.373 & 6.403 & 6.676 \\
$\mathrm{~A}$ & 18.290 & 24.362 & 39.424 & 46.338 & 45.089 \\
\hline
\end{tabular}

Table A.47: Wavelet Power Spectrum Calculations for Muscle 2-CON Subjects 13

\begin{tabular}{cccccc}
\hline & Level 1 & Level 2 & Level 3 & Level 4 & Level 5 \\
\hline $\mathrm{D}$ & 9.798 & 16.314 & 19.753 & 19.312 & 19.549 \\
$\mathrm{~S}$ & 2.041 & 3.398 & 3.510 & 3.975 & 4.553 \\
$\mathrm{~A}$ & 49.894 & 84.482 & 89.872 & 88.383 & 89.000 \\
\hline
\end{tabular}


Table A.48: Wavelet Power Spectrum Calculations for Muscle 2-CON Subjects 14

\begin{tabular}{cccccc}
\hline & Level 1 & Level 2 & Level 3 & Level 4 & Level 5 \\
\hline $\mathrm{D}$ & 10.454 & 12.868 & 17.524 & 19.998 & 20.754 \\
$\mathrm{~S}$ & 0.510 & 0.895 & 3.186 & 5.488 & 5.866 \\
$\mathrm{~A}$ & 2.827 & 4.159 & 6.142 & 6.321 & 6.699 \\
\hline
\end{tabular}

Table A.49: Wavelet Power Spectrum Calculations for Muscle 2-CON Subjects 15

\begin{tabular}{cccccc}
\hline & Level 1 & Level 2 & Level 3 & Level 4 & Level 5 \\
\hline $\mathrm{D}$ & 148.435 & 233.150 & 340.468 & 295.913 & 295.245 \\
$\mathrm{~S}$ & 4.451 & 6.602 & 11.455 & 15.892 & 20.992 \\
$\mathrm{~A}$ & 125.629 & 193.948 & 268.378 & 271.082 & 267.695
\end{tabular}

Table A.50: Wavelet Power Spectrum Calculations for Muscle 2-CON Subjects 16

\begin{tabular}{cccccc}
\hline & Level 1 & Level 2 & Level 3 & Level 4 & Level 5 \\
\hline $\mathrm{D}$ & 10.752 & 14.802 & 21.200 & 27.848 & 26.360 \\
$\mathrm{~S}$ & 1.097 & 1.698 & 2.161 & 2.278 & 2.420 \\
$\mathrm{~A}$ & 10.717 & 15.638 & 22.129 & 23.642 & 22.596 \\
\hline
\end{tabular}




\section{Muscle 2 - OR:}

Tables A.51 - A.62 show the power for muscle 2 of OR group.

Table A.51: Wavelet Power Spectrum Calculations for Muscle 2-OR Subjects 1

\begin{tabular}{cccccc}
\hline & Level 1 & Level 2 & Level 3 & Level 4 & Level 5 \\
\hline $\mathrm{D}$ & 9.166 & 13.820 & 15.052 & 14.768 & 14.948 \\
$\mathrm{~S}$ & 0.640 & 1.361 & 3.681 & 5.734 & 6.262 \\
$\mathrm{~A}$ & 4.393 & 6.175 & 8.715 & 8.910 & 8.826 \\
\hline
\end{tabular}

Table A.52: Wavelet Power Spectrum Calculations for Muscle 2-OR Subjects 2

\begin{tabular}{cccccc}
\hline & Level 1 & Level 2 & Level 3 & Level 4 & Level 5 \\
\hline D & 10.424 & 15.486 & 19.268 & 20.263 & 22.215 \\
S & 3.239 & 5.794 & 6.606 & 8.119 & 8.875 \\
A & 43.230 & 60.027 & 73.495 & 73.003 & 72.547 \\
\hline
\end{tabular}

Table A.53: Wavelet Power Spectrum Calculations for Muscle 2-OR Subjects 3

\begin{tabular}{cccccc}
\hline & Level 1 & Level 2 & Level 3 & Level 4 & Level 5 \\
\hline D & 37.060 & 53.131 & 65.017 & 68.115 & 69.114 \\
S & 9.807 & 14.002 & 24.005 & 31.272 & 32.134 \\
A & 138.355 & 187.669 & 220.631 & 224.287 & 224.146 \\
\hline
\end{tabular}

Table A.54: Wavelet Power Spectrum Calculations for Muscle 2-OR Subjects 4

\begin{tabular}{cccccc}
\hline & Level 1 & Level 2 & Level 3 & Level 4 & Level 5 \\
\hline D & 37.490 & 57.201 & 75.941 & 81.755 & 80.911 \\
S & 3.568 & 6.550 & 11.072 & 15.997 & 22.838 \\
A & 18.086 & 26.652 & 43.978 & 50.092 & 53.315 \\
\hline
\end{tabular}


Table A.55: Wavelet Power Spectrum Calculations for Muscle 2-OR Subjects 5

\begin{tabular}{cccccc}
\hline & Level 1 & Level 2 & Level 3 & Level 4 & Level 5 \\
\hline $\mathrm{D}$ & 24.240 & 34.871 & 44.438 & 80.933 & 74.366 \\
$\mathrm{~S}$ & 0.113 & 0.215 & 0.328 & 0.469 & 0.724 \\
$\mathrm{~A}$ & 3.826 & 4.849 & 8.846 & 14.691 & 13.804 \\
\hline
\end{tabular}

Table A.56: Wavelet Power Spectrum Calculations for Muscle 2-OR Subjects 6

\begin{tabular}{cccccc}
\hline & Level 1 & Level 2 & Level 3 & Level 4 & Level 5 \\
\hline $\mathrm{D}$ & 693.748 & 1203.845 & 1609.567 & 1634.089 & 1780.215 \\
$\mathrm{~S}$ & 62.969 & 100.897 & 171.008 & 252.983 & 288.864 \\
$\mathrm{~A}$ & 613.058 & 1001.713 & 1350.991 & 1264.552 & 1235.940 \\
\hline
\end{tabular}

Table A.57: Wavelet Power Spectrum Calculations for Muscle 2-OR Subjects 7

\begin{tabular}{cccccc}
\hline & Level 1 & Level 2 & Level 3 & Level 4 & Level 5 \\
\hline D & 535.898 & 841.020 & 1113.662 & 1173.318 & 1174.668 \\
S & 168.207 & 284.598 & 509.621 & 626.983 & 640.425 \\
A & 168.908 & 277.864 & 443.648 & 551.551 & 572.558 \\
\hline
\end{tabular}

Table A.58: Wavelet Power Spectrum Calculations for Muscle 2-OR Subjects 8

\begin{tabular}{cccccc}
\hline & Level 1 & Level 2 & Level 3 & Level 4 & Level 5 \\
\hline $\mathrm{D}$ & 8.141 & 11.204 & 19.938 & 18.650 & 18.294 \\
$\mathrm{~S}$ & 3.816 & 6.239 & 15.481 & 19.927 & 25.001 \\
$\mathrm{~A}$ & 10.376 & 15.026 & 28.075 & 32.724 & 32.730 \\
\hline
\end{tabular}

Table A.59: Wavelet Power Spectrum Calculations for Muscle 2-OR Subjects 9

\begin{tabular}{cccccc}
\hline & Level 1 & Level 2 & Level 3 & Level 4 & Level 5 \\
\hline D & 6.581 & 8.447 & 12.402 & 11.712 & 11.499 \\
S & 6.641 & 9.551 & 15.499 & 18.320 & 18.653 \\
A & 9.148 & 13.946 & 19.090 & 18.491 & 18.840 \\
\hline
\end{tabular}


Table A.60: Wavelet Power Spectrum Calculations for Muscle 2-OR Subjects 10

\begin{tabular}{cccccc}
\hline & Level 1 & Level 2 & Level 3 & Level 4 & Level 5 \\
\hline D & 50.262 & 85.738 & 124.497 & 141.650 & 143.019 \\
S & 6.847 & 12.284 & 21.693 & 22.810 & 26.116 \\
A & 21.782 & 26.181 & 34.242 & 46.903 & 67.909 \\
\hline
\end{tabular}

Table A.61: Wavelet Power Spectrum Calculations for Muscle 2-OR Subjects 11

\begin{tabular}{cccccc}
\hline & Level 1 & Level 2 & Level 3 & Level 4 & Level 5 \\
\hline $\mathrm{D}$ & 3.548 & 4.664 & 6.297 & 5.791 & 5.785 \\
$\mathrm{~S}$ & 0.156 & 0.203 & 0.300 & 0.327 & 0.410 \\
$\mathrm{~A}$ & 21.679 & 36.177 & 45.961 & 48.347 & 48.676 \\
\hline
\end{tabular}

Table A.62: Wavelet Power Spectrum Calculations for Muscle 2-OR Subjects 12

\begin{tabular}{cccccc}
\hline & Level 1 & Level 2 & Level 3 & Level 4 & Level 5 \\
\hline D & 42.547 & 59.336 & 118.987 & 119.000 & 129.684 \\
S & 7.721 & 13.829 & 18.217 & 22.227 & 26.778 \\
A & 64.900 & 88.085 & 142.020 & 136.315 & 131.877 \\
\hline
\end{tabular}




\section{Muscle 3 - CON:}

Tables A.63 - A.78 indicate the power values for muscle 3 of CON group.

Table A.63: Wavelet Power Spectrum Calculations for Muscle 3-CON Subjects 1

\begin{tabular}{cccccc}
\hline & Level 1 & Level 2 & Level 3 & Level 4 & Level 5 \\
\hline $\mathrm{D}$ & 497.076 & 822.457 & 808.660 & 800.428 & 795.611 \\
$\mathrm{~S}$ & 2.773 & 4.797 & 7.064 & 8.859 & 10.795 \\
$\mathrm{~A}$ & 162.498 & 260.785 & 342.708 & 331.838 & 333.221
\end{tabular}

Table A.64: Wavelet Power Spectrum Calculations for Muscle 3-CON Subjects 2

\begin{tabular}{cccccc}
\hline & Level 1 & Level 2 & Level 3 & Level 4 & Level 5 \\
\hline D & 326.453 & 534.807 & 644.757 & 637.505 & 632.421 \\
S & 48.383 & 95.583 & 112.166 & 117.314 & 129.691 \\
A & 91.764 & 155.638 & 167.959 & 166.553 & 168.692 \\
\hline
\end{tabular}

Table A.65: Wavelet Power Spectrum Calculations for Muscle 3-CON Subjects 3

\begin{tabular}{cccccc}
\hline & Level 1 & Level 2 & Level 3 & Level 4 & Level 5 \\
\hline $\mathrm{D}$ & 124.233 & 227.332 & 265.344 & 271.912 & 285.654 \\
$\mathrm{~S}$ & 54.618 & 97.620 & 135.054 & 151.085 & 163.683 \\
$\mathrm{~A}$ & 82.432 & 130.924 & 164.939 & 163.833 & 164.820 \\
\hline
\end{tabular}

Table A.66: Wavelet Power Spectrum Calculations for Muscle 3-CON Subjects 4

\begin{tabular}{cccccc}
\hline & Level 1 & Level 2 & Level 3 & Level 4 & Level 5 \\
\hline D & 87.126 & 139.155 & 156.492 & 166.458 & 172.873 \\
S & 175.566 & 277.305 & 306.601 & 344.573 & 365.555 \\
A & 35.767 & 68.836 & 122.789 & 159.686 & 176.755 \\
\hline
\end{tabular}


Table A.67: Wavelet Power Spectrum Calculations for Muscle 3-CON Subjects 5

\begin{tabular}{cccccc}
\hline & Level 1 & Level 2 & Level 3 & Level 4 & Level 5 \\
\hline $\mathrm{D}$ & 36.427 & 55.111 & 84.935 & 92.454 & 89.458 \\
$\mathrm{~S}$ & 6.363 & 12.497 & 15.455 & 17.910 & 22.549 \\
$\mathrm{~A}$ & 34.615 & 52.456 & 81.618 & 80.382 & 77.213
\end{tabular}

Table A.68: Wavelet Power Spectrum Calculations for Muscle 3-CON Subjects 6

\begin{tabular}{cccccc}
\hline & Level 1 & Level 2 & Level 3 & Level 4 & Level 5 \\
\hline D & 178.423 & 287.304 & 375.901 & 364.661 & 353.847 \\
S & 20.020 & 47.380 & 103.189 & 152.773 & 190.989 \\
A & 138.541 & 207.449 & 361.070 & 356.026 & 368.845 \\
\hline
\end{tabular}

Table A.69: Wavelet Power Spectrum Calculations for Muscle 3-CON Subjects 7

\begin{tabular}{cccccc}
\hline & Level 1 & Level 2 & Level 3 & Level 4 & Level 5 \\
\hline D & 703.160 & 1136.565 & 1664.831 & 1531.623 & 1523.088 \\
S & 80.780 & 143.932 & 229.145 & 274.731 & 335.666 \\
A & 638.971 & 1006.164 & 1181.079 & 1243.186 & 1235.578 \\
\hline
\end{tabular}

Table A.70: Wavelet Power Spectrum Calculations for Muscle 3-CON Subjects 8

\begin{tabular}{cccccc}
\hline & Level 1 & Level 2 & Level 3 & Level 4 & Level 5 \\
\hline D & 391.437 & 676.167 & 1092.431 & 1305.961 & 1367.200 \\
S & 189.154 & 340.897 & 472.470 & 554.419 & 694.553 \\
A & 465.481 & 803.266 & 991.217 & 965.769 & 992.540 \\
\hline
\end{tabular}

Table A.71: Wavelet Power Spectrum Calculations for Muscle 3-CON Subjects 9

\begin{tabular}{cccccc}
\hline & Level 1 & Level 2 & Level 3 & Level 4 & Level 5 \\
\hline D & 155.602 & 250.867 & 294.101 & 336.021 & 347.326 \\
S & 69.496 & 102.568 & 134.358 & 132.356 & 142.275 \\
A & 47.149 & 85.434 & 103.584 & 113.534 & 121.371 \\
\hline
\end{tabular}


Table A.72: Wavelet Power Spectrum Calculations for Muscle 3-CON Subjects 10

\begin{tabular}{cccccc}
\hline & Level 1 & Level 2 & Level 3 & Level 4 & Level 5 \\
\hline D & 625.449 & 1063.203 & 1146.037 & 1168.231 & 1171.506 \\
S & 68.033 & 149.599 & 161.139 & 174.247 & 176.196 \\
A & 138.174 & 226.250 & 359.235 & 432.702 & 441.502 \\
\hline
\end{tabular}

Table A.73: Wavelet Power Spectrum Calculations for Muscle 3-CON Subjects 11

\begin{tabular}{cccccc}
\hline & Level 1 & Level 2 & Level 3 & Level 4 & Level 5 \\
\hline D & 560.791 & 955.831 & 1058.664 & 1020.265 & 1014.233 \\
S & 1048.788 & 1763.070 & 2166.920 & 2198.518 & 2242.857 \\
A & 157.841 & 231.215 & 259.253 & 259.598 & 267.300 \\
\hline
\end{tabular}

Table A.74: Wavelet Power Spectrum Calculations for Muscle 3-CON Subjects 12

\begin{tabular}{cccccc}
\hline & Level 1 & Level 2 & Level 3 & Level 4 & Level 5 \\
\hline D & 1158.028 & 1934.565 & 3208.305 & 3047.031 & 3050.432 \\
S & 314.863 & 574.048 & 940.482 & 1487.251 & 1746.079 \\
A & 371.082 & 564.203 & 846.181 & 807.819 & 827.573 \\
\hline
\end{tabular}

Table A.75: Wavelet Power Spectrum Calculations for Muscle 3-CON Subjects 13

\begin{tabular}{cccccc}
\hline & Level 1 & Level 2 & Level 3 & Level 4 & Level 5 \\
\hline D & 625.975 & 995.065 & 1275.756 & 1166.583 & 1169.189 \\
S & 74.664 & 132.690 & 179.696 & 218.048 & 287.688 \\
A & 317.583 & 551.739 & 820.001 & 871.994 & 877.837 \\
\hline
\end{tabular}

Table A.76: Wavelet Power Spectrum Calculations for Muscle 3-CON Subjects 14

\begin{tabular}{cccccc}
\hline & Level 1 & Level 2 & Level 3 & Level 4 & Level 5 \\
\hline D & 189.419 & 285.742 & 312.286 & 324.986 & 338.000 \\
S & 37.167 & 105.513 & 166.226 & 184.567 & 219.616 \\
A & 69.331 & 125.254 & 226.561 & 281.302 & 288.809 \\
\hline
\end{tabular}


Table A.77: Wavelet Power Spectrum Calculations for Muscle 3-CON Subjects 15

\begin{tabular}{cccccc}
\hline & Level 1 & Level 2 & Level 3 & Level 4 & Level 5 \\
\hline $\mathrm{D}$ & 71.660 & 114.217 & 155.192 & 153.889 & 154.763 \\
$\mathrm{~S}$ & 1.351 & 2.280 & 4.045 & 5.964 & 7.392 \\
$\mathrm{~A}$ & 64.100 & 102.363 & 144.907 & 126.139 & 126.353
\end{tabular}

Table A.78: Wavelet Power Spectrum Calculations for Muscle 3-CON Subjects 16

\begin{tabular}{cccccc}
\hline & Level 1 & Level 2 & Level 3 & Level 4 & Level 5 \\
\hline D & 623.189 & 1029.776 & 1299.165 & 1269.022 & 1265.944 \\
S & 198.519 & 302.462 & 629.635 & 836.473 & 953.208 \\
A & 571.017 & 937.823 & 1089.158 & 1091.524 & 1124.257
\end{tabular}




\section{Muscle 3 - OR:}

Tables A.79 - A.90 show the power values for muscle 3 of OR group.

Table A.79: Wavelet Power Spectrum Calculations for Muscle 3-OR Subjects 1

\begin{tabular}{cccccc}
\hline & Level 1 & Level 2 & Level 3 & Level 4 & Level 5 \\
\hline D & 241.137 & 405.907 & 468.854 & 476.776 & 470.264 \\
S & 55.161 & 92.033 & 160.331 & 189.950 & 199.877 \\
A & 905.491 & 1494.067 & 1849.520 & 1746.178 & 1732.128 \\
\hline
\end{tabular}

Table A.80: Wavelet Power Spectrum Calculations for Muscle 3-OR Subjects 2

\begin{tabular}{cccccc}
\hline & Level 1 & Level 2 & Level 3 & Level 4 & Level 5 \\
\hline D & 209.650 & 356.795 & 366.922 & 351.304 & 350.055 \\
S & 65.453 & 135.278 & 210.988 & 261.701 & 292.567 \\
A & 400.546 & 605.973 & 723.068 & 818.077 & 823.966 \\
\hline
\end{tabular}

Table A.81: Wavelet Power Spectrum Calculations for Muscle 3-OR Subjects 3

\begin{tabular}{cccccc}
\hline & Level 1 & Level 2 & Level 3 & Level 4 & Level 5 \\
\hline $\mathrm{D}$ & 1.493 & 1.317 & 1.876 & 1.917 & 1.902 \\
$\mathrm{~S}$ & 0.371 & 0.337 & 0.466 & 0.483 & 0.497 \\
$\mathrm{~A}$ & 5.515 & 7.326 & 11.799 & 11.818 & 11.417 \\
\hline
\end{tabular}

Table A.82: Wavelet Power Spectrum Calculations for Muscle 3-OR Subjects 4

\begin{tabular}{cccccc}
\hline & Level 1 & Level 2 & Level 3 & Level 4 & Level 5 \\
\hline D & 821.632 & 1518.069 & 1669.291 & 1717.896 & 1766.572 \\
S & 187.145 & 514.404 & 1105.047 & 1480.271 & 1524.736 \\
A & 188.328 & 312.572 & 491.248 & 634.088 & 657.874 \\
\hline
\end{tabular}


Table A.83: Wavelet Power Spectrum Calculations for Muscle 3-OR Subjects 5

\begin{tabular}{cccccc}
\hline & Level 1 & Level 2 & Level 3 & Level 4 & Level 5 \\
\hline D & 545.719 & 865.051 & 1103.135 & 1005.195 & 978.251 \\
S & 37.543 & 73.965 & 195.079 & 296.906 & 342.106 \\
A & 160.038 & 233.738 & 334.886 & 396.874 & 418.170
\end{tabular}

Table A.84: Wavelet Power Spectrum Calculations for Muscle 3-OR Subjects 6

\begin{tabular}{cccccc}
\hline & Level 1 & Level 2 & Level 3 & Level 4 & Level 5 \\
\hline D & 261.641 & 464.855 & 579.377 & 562.829 & 567.709 \\
S & 39.149 & 68.106 & 116.491 & 145.394 & 196.061 \\
A & 346.235 & 619.076 & 819.979 & 790.901 & 812.253 \\
\hline
\end{tabular}

Table A.85: Wavelet Power Spectrum Calculations for Muscle 3-OR Subjects 7

\begin{tabular}{cccccc}
\hline & Level 1 & Level 2 & Level 3 & Level 4 & Level 5 \\
\hline D & 146.493 & 241.115 & 258.398 & 248.978 & 248.341 \\
S & 16.975 & 31.099 & 27.700 & 28.182 & 31.674 \\
A & 82.070 & 133.048 & 152.403 & 152.109 & 151.216 \\
\hline
\end{tabular}

Table A.86: Wavelet Power Spectrum Calculations for Muscle 3-OR Subjects 8

\begin{tabular}{cccccc}
\hline & Level 1 & Level 2 & Level 3 & Level 4 & Level 5 \\
\hline D & 272.998 & 487.699 & 568.959 & 546.757 & 556.395 \\
S & 191.790 & 393.960 & 535.219 & 600.226 & 705.901 \\
A & 200.804 & 279.040 & 353.518 & 346.098 & 360.644 \\
\hline
\end{tabular}

Table A.87: Wavelet Power Spectrum Calculations for Muscle 3-OR Subjects 9

\begin{tabular}{cccccc}
\hline & Level 1 & Level 2 & Level 3 & Level 4 & Level 5 \\
\hline D & 101.237 & 199.715 & 202.218 & 204.034 & 205.073 \\
S & 127.145 & 209.465 & 240.019 & 249.851 & 257.056 \\
A & 92.910 & 154.030 & 162.055 & 175.929 & 183.816 \\
\hline
\end{tabular}


Table A.88: Wavelet Power Spectrum Calculations for Muscle 3-OR Subjects 10

\begin{tabular}{cccccc}
\hline & Level 1 & Level 2 & Level 3 & Level 4 & Level 5 \\
\hline D & 656.948 & 1091.735 & 1358.654 & 1369.095 & 1362.085 \\
S & 59.806 & 114.845 & 241.148 & 369.472 & 387.917 \\
A & 208.228 & 326.177 & 407.325 & 401.511 & 399.296 \\
\hline
\end{tabular}

Table A.89: Wavelet Power Spectrum Calculations for Muscle 3-OR Subjects 11

\begin{tabular}{cccccc}
\hline & Level 1 & Level 2 & Level 3 & Level 4 & Level 5 \\
\hline $\mathrm{D}$ & 0.900 & 0.844 & 1.243 & 1.270 & 1.321 \\
$\mathrm{~S}$ & 0.150 & 0.166 & 0.288 & 0.372 & 0.430 \\
$\mathrm{~A}$ & 4.821 & 6.022 & 9.874 & 10.793 & 9.689
\end{tabular}

Table A.90: Wavelet Power Spectrum Calculations for Muscle 3-OR Subjects 12

\begin{tabular}{cccccc}
\hline & Level 1 & Level 2 & Level 3 & Level 4 & Level 5 \\
\hline $\mathrm{D}$ & 15.952 & 19.053 & 39.267 & 39.485 & 39.550 \\
$\mathrm{~S}$ & 1.500 & 2.011 & 5.307 & 7.370 & 7.729 \\
$\mathrm{~A}$ & 14.054 & 14.865 & 23.447 & 25.729 & 25.945 \\
\hline
\end{tabular}




\section{Ratio of Ascending Over Descending Phase}

\section{Muscle 1 - CON:}

Table A.91: Ratio of A/D for Muscle 1 of CON subjects

\begin{tabular}{cccccc}
\hline Subject & Level 1 & Level 2 & Level 3 & Level 4 & Level 5 \\
\hline 1 & 3.822 & 3.692 & 5.147 & 5.686 & 5.454 \\
2 & 7.136 & 8.070 & 10.591 & 8.993 & 9.057 \\
3 & 9.687 & 10.526 & 12.238 & 9.942 & 9.246 \\
4 & 29.914 & 32.168 & 34.478 & 40.984 & 40.771 \\
5 & 14.984 & 13.621 & 15.911 & 13.421 & 12.982 \\
6 & 7.172 & 6.841 & 8.169 & 7.833 & 6.791 \\
7 & 2.455 & 2.362 & 2.525 & 2.630 & 2.529 \\
8 & 10.909 & 11.108 & 11.973 & 16.073 & 14.527 \\
9 & 6.152 & 6.483 & 7.326 & 5.648 & 5.016 \\
10 & 7.192 & 5.963 & 7.277 & 8.883 & 8.596 \\
11 & 4.258 & 6.940 & 7.642 & 8.619 & 8.018 \\
12 & 20.389 & 24.169 & 26.338 & 23.739 & 23.671 \\
13 & 2.271 & 2.140 & 2.702 & 3.623 & 3.589 \\
14 & 55.605 & 72.296 & 81.603 & 68.821 & 56.772 \\
15 & 1.989 & 1.909 & 2.158 & 2.077 & 1.835 \\
16 & 1.405 & 1.412 & 1.124 & 1.145 & 1.124 \\
\hline
\end{tabular}




\section{Muscle 1 - OR:}

Table A.92: Ratio of A/D for Muscle 1 of OR subjects

\begin{tabular}{cccccc}
\hline Subject & Level 1 & Level 2 & Level 3 & Level 4 & Level 5 \\
\hline 1 & 2.140 & 2.071 & 2.887 & 2.599 & 2.535 \\
2 & 45.068 & 45.246 & 64.179 & 74.109 & 66.716 \\
3 & 0.966 & 1.054 & 1.038 & 0.946 & 0.955 \\
4 & 4.508 & 4.738 & 6.686 & 7.391 & 7.456 \\
5 & 7.661 & 7.050 & 8.958 & 12.441 & 12.242 \\
6 & 4.750 & 4.724 & 5.290 & 5.148 & 5.170 \\
7 & 21.163 & 24.523 & 24.822 & 26.346 & 25.905 \\
8 & 11.889 & 11.280 & 14.491 & 12.108 & 11.631 \\
9 & 2.518 & 2.662 & 2.560 & 2.562 & 2.724 \\
10 & 4.857 & 5.148 & 7.397 & 7.160 & 6.638 \\
11 & 5.533 & 7.028 & 5.764 & 6.464 & 6.655 \\
12 & 0.545 & 0.560 & 0.501 & 0.532 & 0.469 \\
\hline
\end{tabular}


Muscle 2 - CON:

Table A.93: Ratio of A/D for Muscle 2 of CON subjects

\begin{tabular}{cccccc}
\hline Subject & Level 1 & Level 2 & Level 3 & Level 4 & Level 5 \\
\hline 1 & 0.210 & 0.211 & 0.224 & 0.277 & 0.302 \\
2 & 0.107 & 0.098 & 0.093 & 0.106 & 0.097 \\
3 & 0.394 & 0.363 & 0.371 & 0.344 & 0.314 \\
4 & 0.533 & 0.536 & 0.705 & 0.854 & 0.953 \\
5 & 5.426 & 4.813 & 4.959 & 4.954 & 4.643 \\
6 & 0.391 & 0.362 & 0.366 & 0.303 & 0.310 \\
7 & 1.661 & 1.700 & 1.441 & 1.415 & 1.405 \\
8 & 1.408 & 1.503 & 1.737 & 2.343 & 3.441 \\
9 & 1.665 & 1.570 & 1.739 & 1.678 & 1.676 \\
10 & 0.836 & 0.742 & 0.921 & 0.979 & 1.041 \\
11 & 0.667 & 0.626 & 0.674 & 0.780 & 0.806 \\
12 & 0.674 & 0.713 & 0.778 & 0.823 & 0.749 \\
13 & 5.092 & 5.179 & 4.550 & 4.577 & 4.553 \\
14 & 0.270 & 0.323 & 0.351 & 0.316 & 0.323 \\
15 & 0.846 & 0.832 & 0.788 & 0.916 & 0.907 \\
16 & 0.997 & 1.056 & 1.044 & 0.849 & 0.857 \\
\hline
\end{tabular}


Muscle 2 - OR:

Table A.94: Ratio of A/D for Muscle 2 of OR subjects

\begin{tabular}{cccccc}
\hline Subject & Level 1 & Level 2 & Level 3 & Level 4 & Level 5 \\
\hline 1 & 0.479 & 0.447 & 0.579 & 0.603 & 0.590 \\
2 & 4.147 & 3.876 & 3.814 & 3.603 & 3.266 \\
3 & 3.733 & 3.532 & 3.393 & 3.293 & 3.243 \\
4 & 0.482 & 0.466 & 0.579 & 0.613 & 0.659 \\
5 & 0.158 & 0.139 & 0.199 & 0.182 & 0.186 \\
6 & 0.884 & 0.832 & 0.839 & 0.774 & 0.694 \\
7 & 0.315 & 0.330 & 0.398 & 0.470 & 0.487 \\
8 & 1.275 & 1.341 & 1.408 & 1.755 & 1.789 \\
9 & 1.390 & 1.651 & 1.539 & 1.579 & 1.638 \\
10 & 0.433 & 0.305 & 0.275 & 0.331 & 0.475 \\
11 & 6.110 & 7.757 & 7.299 & 8.349 & 8.414 \\
12 & 1.525 & 1.485 & 1.194 & 1.146 & 1.017 \\
\hline
\end{tabular}




\section{Muscle 3 - CON:}

Table A.95: Ratio of A/D for Muscle 3 of CON subjects

\begin{tabular}{cccccc}
\hline Subject & Level 1 & Level 2 & Level 3 & Level 4 & Level 5 \\
\hline 1 & 0.327 & 0.317 & 0.424 & 0.415 & 0.419 \\
2 & 0.281 & 0.291 & 0.260 & 0.261 & 0.267 \\
3 & 0.664 & 0.576 & 0.621 & 0.603 & 0.577 \\
4 & 0.411 & 0.495 & 0.785 & 0.959 & 1.022 \\
5 & 0.950 & 0.952 & 0.961 & 0.869 & 0.863 \\
6 & 0.776 & 0.722 & 0.961 & 0.976 & 1.042 \\
7 & 0.909 & 0.885 & 0.709 & 0.812 & 0.811 \\
8 & 1.189 & 1.188 & 0.907 & 0.740 & 0.726 \\
9 & 0.303 & 0.341 & 0.352 & 0.338 & 0.349 \\
10 & 0.221 & 0.213 & 0.313 & 0.370 & 0.377 \\
11 & 0.281 & 0.242 & 0.245 & 0.254 & 0.264 \\
12 & 0.320 & 0.292 & 0.264 & 0.265 & 0.271 \\
13 & 0.507 & 0.554 & 0.643 & 0.747 & 0.751 \\
14 & 0.366 & 0.438 & 0.725 & 0.866 & 0.854 \\
15 & 0.894 & 0.896 & 0.934 & 0.820 & 0.816 \\
16 & 0.916 & 0.911 & 0.838 & 0.860 & 0.888 \\
\hline
\end{tabular}




\section{Muscle 3 - OR:}

Table A.96: Ratio of A/D for Muscle 3 of OR subjects

\begin{tabular}{cccccc}
\hline Subject & Level 1 & Level 2 & Level 3 & Level 4 & Level 5 \\
\hline 1 & 3.755 & 3.681 & 3.945 & 3.662 & 3.683 \\
2 & 1.911 & 1.698 & 1.971 & 2.329 & 2.354 \\
3 & 3.695 & 5.562 & 6.291 & 6.164 & 6.003 \\
4 & 0.229 & 0.206 & 0.294 & 0.369 & 0.372 \\
5 & 0.293 & 0.270 & 0.304 & 0.395 & 0.427 \\
6 & 1.323 & 1.332 & 1.415 & 1.405 & 1.431 \\
7 & 0.560 & 0.552 & 0.590 & 0.611 & 0.609 \\
8 & 0.736 & 0.572 & 0.621 & 0.633 & 0.648 \\
9 & 0.918 & 0.771 & 0.801 & 0.862 & 0.896 \\
10 & 0.317 & 0.299 & 0.300 & 0.293 & 0.293 \\
11 & 5.360 & 7.137 & 7.946 & 8.498 & 7.335 \\
12 & 0.881 & 0.780 & 0.597 & 0.652 & 0.656
\end{tabular}




\section{References}

[1] "Femoroacetabular Impingement (FAI)," American academy of orthopaedic surgeons, March 11, 2014. Available at: http://orthoinfo.aaos.org/topic.cfm?topic=A00571.

[2] D. Q. Thomas, "Muscle at the Hip," March, 10, 2014. Available at: http://www.castonline.ilstu.edu/Thomas/181/Muscles

[3] M. J. Keogh and M. E. Batt, "A Review of Femoroacetabular Impingement in Athletes," Sport Med, vol. 38(10), pp. 863-878, 2008.

[4] O. College, "Anatomy \& Physiology [Connexions Web site]," March 11, 2014. Available at: http://cnx.org/content/m46476/latest/?collection=col11496/latest.

[5] O. Rioul and M. Vetterli, "Wavelet and Signal Processing," IEEE Signal Process Magazine, vol. 8, pp. 14-38, 1991.

[6] M. Lamontagne, M. J. Kennedy, and P. E. Beaule, "The effect of cam FAI on hip and pelvic motion during maximum squat," Clinical Orthopaedics and Related Research, vol. 467(3), pp. 645-650, 2009. 
[7] J. B. Samora, V. Y. Ng, and T. J. Ellis, "Femoroacetabular Impingement: A Common Cause of Hip Pain in Young Adults," Clinical Journal of Sport Medicine, vol. 21(1), pp. 51-56, 2011.

[8] S. J. Wisniewski and B. Grogg, "Femoroacetabular Impingement: An Overlooked Cause of Hip Pain," American Journal of Physical Medicine \& Rehabilitation, vol. 85(6), pp. 546-549, 2006.

[9] M. B. I. Reaz, M. S. Hussain, and F. Mohd-Yasin, "Techniques of EMG Signal Analysis: Detection, Processing, Classification and Applications," Biological Procedures Online, vol. 8(1), pp. 11-35, 2006.

[10] S. Ahmed, S. Ahmed, M. O. Faruqe, and M. R. Islam, "EMG Signal Decomposition Using Wavelet Transformation with Respect to Different Wavelet and a Comparative Study," Life and Medical Sciences, pp. 730-735, 2009.

[11] C. A. Myers, B. C. Register, P. Lertwanich, L. Ejnisman, W. W. Pennington, J. E. Giphart, R. F. LaPrade, and M. J. Philippon, "Role of the Acetabular Labrum and the Iliofemoral Ligament in Hip Stability," The American Journal of Sports Medicine, vol. 39(1), pp. 85S-91S, 2011.

[12] H. D. Martin, A. Savage, B. A. Braly, I. J. Palmer, D. P. Beall, and B. Kelly, "The Function of the Hip Capsular Ligaments: A Quantitative Report," The Journal of Arthroscopic and Related Surgery, vol. 24(2), pp. 188-195, 2008.

[13] S. R. Myers, H. Eijer, and R. Ganz, "Anterior Femoroacetabular Impingement After Periacetabular Osteotomy," Clinical Orthopaedics $\&$ Related Research, vol. 363, pp. 93-99, 1999. 
[14] F. M. Jaberi and J. Parvizi, "Hip Pain in Young Adults," The Journal of Arthroplasty, vol. 22(7), pp. 37-42, 2007.

[15] C. GM., The Cell: A Molecular Approach. 2nd edition. Sunderland (MA): Sinauer Associates, 2000.

[16] J. V. Basmajian and C. J. D. Luca, Muscle Alive: Their Functions Revealed by Electromyography, 5th ed. Williams \& Wilkins, 1985.

[17] R. Merletti and P. Parker, Eds., Electromyography: Physiology, Engineering, and Noninvasive Applications. John Wiley \& Sons, Inc., Hoboken, New Jersey., 2004.

[18] P. Konrad, "The ABC of EMG - A Practical Introduction to Kinesiological EMG," Power by Noraxon INC. USA, Version 1.0 April 2005, pp. 5.

[19] J. S. Karlsson, B. Gerdle, and M. Akay, "Analyzing Surface Myoelectric Signal Recorded During Isokinetic Contractions: A Time-Frequency Approach Using Wavelet to Study Movements at Different Angular Velocities," IEEE Engineering in Medicine and Biology, pp. 97-105, 2001.

[20] N. C. Casartelli, N. A. Maffiuletti, J. Item-Glatthorn, S. Staehli, M. Bizzini, F. M. Impellizzeri, and M. Leunig, "Hip Muscle Weakness in Patients with Symptomatic Femoroacetabular Impingement," Osteoarthrities and Cartilage, vol. 19, pp. 816-821, 2011.

[21] N. C. Casartelli, M. Leunig, J. F. Item-Glatthorn, R. Lepers, and N. A. Maffiuletti, "Hip Flexor Muscle Fatigue in Patients with Symptomatic Femoroacetab- 
ular Impingement," International Orthopaedics (SICOT), vol. 36, pp. 967-973, 2012.

[22] G. Mantovani, M. Lamontagne, K. Dwyer, and P. Beaule, "Muscular CoActivation in Subjects Affected by Femoroacetabular Impingement," in 30th Annual Conference of Biomechanics in Sports, Melbourne, 2012.

[23] S. Karlsson, J. Yu, and M. Akay, "Time-Frequency Analysis of Myoelectric Signal During Dynamic Contractions: A Comparative Study," IEEE Transactions on Biomedical Engineering, vol. 47(2), pp. 228-238, 2000.

[24] D. Gabor, "Theory of communication," J. IEEE, vol. 93, pp. 429-457, 1946.

[25] T. A. C. M. Classen and W. F. G. Mecklenbrauke, "The Wigner DistributionA Tool for Time-Frequency Analysis: Part 1: Continuous-Time Signals," Philips J. Res., vol. 35, pp. 217-250, 1980.

[26] J. Duchene, D. Devedeux, S. Mansour, and C. Marque, "Analyzing Uterine EMG: Tracking Instantaneous Burst Frequency," IEEE Engineering in Medicine and Biology, vol. 14, pp. 125-132, 1995.

[27] M. Knaflitz and P. Bonato, "Time-Frequency Methods Applied to Muscle Fatigue Assessment During Dynamic Contractions," Journal of Electromyography and Kinesiology, vol. 9, pp. 337-350, 1999.

[28] P. Bonato, G. Gagliati, and M. Knaflitz, "Analysis of Myoelectric Signal Recorded During Dynamic Contractions: A Time-Frequency Approach to As- 
sessing Muscle Fatigue," IEEE Engineering in Medicine and Biology, vol. 15, pp. 102-111, 1996.

[29] Y. Meyer, Wavelets Algorithms and Applications. Philadelphia: Society of Industrial and Applied Mathematics, 1993.

[30] L. Debnath, "Brief Historical Introduction to Wavelet Transforms," International Journal of Mathematical Education in Science and Technology, vol. 29(5), pp. 677-688, 1998.

[31] V. von Tscharner, "Intensity Analysis in Time-Frequency Space of Surface Myoelectric signal by Wavelet of Specified Resolution," Journal of Electromyography and Kinesiology, vol. 10, pp. 433-445, 2000.

[32] S. Karlsson, J. Yu, and M. Akay, "Enhancement of Spectral Analysis of Myoelectric SSignal During Static Contractions Using Wavelet Methods," IEEE Transaction on Biomedical Engineering, vol. 46(6), pp. 670-684, 1999.

[33] M. Barandun, V. von Tscharner, C. Meuli-Simmen, V. Bowen, and V. Valderrabano, "Frequency and Conduction Velocity Analysis of the Abductor Policis Braevis Muscle During Early Fatigue," Journal of Electromyography and Kinesiology, vol. 19, pp. 65-74, 2009.

[34] N. Sobahi, "Denoising of EMG Signal Based on Wavelet Transform," Asian Transactions on Engineering, vol. 1(5), pp. 17-23, 2011.

[35] G. Strang and T. Nguyen, Wavelets and Filter Banks. Wellesley, Massachusetts: Wellesley-Cambridge Press, 1997. 
[36] J. Frere, B. Gopfert, J. Slawinski, and C. Tourny-Chollet, "Influence of the Scale Function on Wavelet Transformation of the Surface Electromyographic Signal," Computer Methods in Biomechanics and Biomedical Engineering, vol. 15(2), pp. 111-120, 2012.

[37] S. Santoso, E. J. Powers, and W. M. Grady, "Electric Power Quality Disturbance Detection Using Wavelet Transform Analysis," IEEE, pp. 166-169, 1994.

[38] J. S. Walker, A Primer on Wavelets and Their Scientific Applications. Boca Raton, FL: CRC, 1999.

[39] J. Karlsson, N. Ostlundm, B. Larsson, and B. Gerdle, "An Estimation of the Influence of Force Decrease on the Mean Power Spectral Frequency Shift of the EMG During Repetitive Maximum Dynamic Knee Extensions," Journal of Electromyography and Kinesiology, vol. 13, pp. 461-468, 2003.

[40] O. P. Neto, M. Magini, and M. T. Pacheco, "Electromyographic Study of a Sequence of Yau-Man Kung Fu Palm Strikes With and Without Impact," Journal of Sports Science and Medicine, vol. 6, pp. 23-27, 2007.

[41] I. Hostens, S. J., A. Spaepen, and H. Ramon, "Validation of the wavelet spectral estimation technique in Biceps Brachii and Brachioradialis fatigue assessment during prolonged low-level static and dynamic contractions," Journal of Electromyography and Kinesiology, vol. 14, pp. 205-215, 2004.

[42] T. W. Beck, T. J. Housh, G. O. Johnson, J. P. Weir, J. T. Cramer, J. W. Coburn, and M. H. Malek, "Comparison of Fourier and Wavelet Transform Procedures for Examining the Mechanomyographic and Electromyographic Frequency Domain 
Responses During Fatiguing Isokinetic Muscle Actions of the Biceps Brachii," Journal of Electromyography and Kinesiology, vol. 15, pp. 190-199, 2005.

[43] O. Neto and M. Magini, "Electromiographic and Kinematic Characteristics of Kung Fu Yau-Man Palm Strike," Journal of Electromyography and Kinesiology, vol. 18, pp. 1047-1052, 2008.

[44] V. von Tscharner, "Time-Frequency and Principal-Component Methods for the Analysis of EMG Recorded During a Mildly Fatiguing Exercise on a Cycle Ergometer," Journal of Electromyography and Kinesiology, vol. 12, pp. 479-492, 2002.

[45] V. von Tscharner and B. Goepfert, "Gender Dependent EMGs of Runners Resolved by Time/Frequency and Principal Pattern Analysis," Journal of Electromyography and Kinesiology, vol. 13, pp. 253-272, 2003.

[46] V. von Tscharner, B. Goepfert, and B. M. Nigg, "Changes in EMG Signals for the Muscle Tibialis Anterior while Running Barefoot or with Shoes Resolved by Non-Linearly Scaled Wavelets," Journal of Biomechanics, vol. 36, pp. 1169-1176, 2003.

[47] V. von Tscharner and B. Goepfert, "Estimation of the Interplay Between Groups of Fast and Slow Muscle Fibers of the Tibialis Anterior and Gastrocnemius Muscle While Running," Journal of Electromyography and Kinesiology, vol. 16, pp. 188-197, 2006.

[48] M. Flanders, "Transform Wavelet for single-trial EMG," Journal of Neuroscience Methods, vol. 116, pp. 165-177, 2002. 
[49] J. Kilby and H. Gholam Hosseini, "Wavelet Analysis of Surface Electromyography Signals," in Proceedings of the 26th Annual International Conference of the IEEE EMBS San Francisco, CA, USA, September 1-5, 2004.

[50] P. Guglielminotti and R. Merletti, Effect of Electrode Location on Surface Myoelectric Signal Variables: A simulation Study. 9th Int. Congress of ISEK, Florence, Italy, 1992.

[51] C. S. Pattichis and M. S. Pattichis, "Time-Scale Analysis of Motor Unit Action Potentials," IEEE Transactions on Biomedical Engineering, vol. 46(11), pp. 1320-1329, 1999.

[52] F. Laterza and G. Olmo, "Analysis of EMG Signals by Mean of the Matched Wavelet Transform," Electronics Letters, vol. 33(5), pp. 357-359, 1997.

[53] D. Zennaro, P. Wellig, V. M. Koch, G. S. Moschytz, and L. Thomas, "A Software Package for the Decomposition of Long-Term Multichannel EMG Signals Using Wavelet Coefficients," IEEE Transaction on Biomedical Engineering, vol. 50(1), pp. 58-69, 2003.

[54] R. Yamada, J. Ushiba, Y. Tomita, and Y. Masakado, "Decomposition of Electromyographic Signal by Principal Component Analysis of Wavelet Coefficients," IEEE EMBS Asian-Pacific Conference on Biomedical Engineering, Keihanna, Japan, pp. 118-119, 2003.

[55] C. J. D. Luca, "Myoelectric Manifestations of Localized Muscular Fatigue in Humans," Critical Review in Biomedical Engineering, vol. 11(4), pp. 251-279, 1984. 
[56] M. Cifreka, V. Medved, S. Tonkovic, and S. Ostojic, "Surface EMG based muscle fatigue evaluation in biomechanics," Clinical Biomechanics, vol. 24(4), pp. 327$340,2009$.

[57] F. B. Stulen and C. J. D. Luca, "Frequency Parameters of the Myoelectric Signal as a Measure of Muscle Conduction Velocity," IEEE Transaction on Biomedical Engineering, vol. 28(7), pp. 515-523, 1981.

[58] S. Thongpanja, A. Phinyomark, P. Phukpattaranont, and C. Limsakul, "Mean and Median Frequency of EMG Signal to Determine Muscle Force based on Time-Dependent Power Spectrum," Elektronika IR Electrotechnika, vol. 19(3), pp. 51-56, 2013.

[59] M. Knaflitz and P. Bonato, "Time-Frequency Methods Applied to Muscle Fatigue Assessment during Dynamic Contractions," Journal of Electromyography and Kinesiology, vol. 9, pp. 337-350, 1999.

[60] I. Nakajima, "Fast Fourier Transform Analysis of the Masseter Muscle EMG during Reaction to a Warning Signal," Electromyogr Clin Neurophysiol., vol. 35(5), pp. 281-4, 1995.

[61] K. Seki, Y. Miyazaki, M. Watanabe, A. Nagata, and M. Narusawa, "Surface Electromyogram Spectral Characterization and Motor Unit Activity During Voluntary Ramp Contraction in Men," Eur J Appl Physiol Occup Physiol, vol. 63, pp. 165-72, 1991.

[62] R. Constable and R. J. Thornhill, "Using the Discrete Wavelet Transform for 
Time-Frequency Analysis of the Surface EMG Signal," Biomed Sci. Instrum., vol. 29, pp. 121-7, 1993.

[63] S. Karlsson and B. Gerdle, "Mean Frequency and Signal amplitude of the Surface EMG of the Quadriceps Muslces Increase with Increasing Torque - a Study Using the Continuous Wavelet Transform," Journal of Electromyography and Kinesiology, vol. 11, pp. 131-140, 2001.

[64] S. Kumar, Y. Narayan, and T. Amell, "Spectral Profile of Superficial Cervical Muscles," J Electromyogr Kinesiol, vol. 11, pp. 269-80, 2001.

[65] D. K. Kumar, N. D. Pah, and A. Bradley, "Wavelet Analysis of Surface Electromyography to Determine Muscle Fatigue," IEEE Transaction on Neural Systems and Rehabilitation Engineering, vol. 11(4), pp. 400-406, 2003.

[66] R. T. Lauer, C. Stackhouse, P. A. Shewokis, B. T. Smith, M. Orlin, and J. J. McCarthy, "Assessment of Wavelet Analysis of Gait in Children with Typical Development and Cerebral Palsy," Journal of Biomechanics, vol. 38, pp. 1351$1357,2005$.

[67] R. Constable and R. J. Thornhill, "Time-Frequency Analysis of the Surface EMG During Maximum Height Jumps Under Altered-G Conditions," Biomed Sci. Instrum., vol. 30, pp. 69-74, 1994.

[68] N. H. Gheab and S. N. Saleem, "Comparison Study of Electromyography Using Wavelet and Neural Network," Al-Khwarizmi Engineering Journal, vol. 4(3), pp. 108-119, 2008. 
[69] M. Ozsert, O. Yavuz, and L. Durak-Ata, "Analysis and Classification of Compressed EMG Signals by Wavelet Transform via Alternative Neural Networks Algorithms," Computer Methods in Biomechanics and Biomedical Engineering, vol. 14(6), pp. 521-525, 2011.

[70] V. von Tscharner, "Spherical Classification of Wavelet Transform EMG Intensity Patterns," Journal of Electromyography and Kinesiology, vol. 19, pp. e334-e344, 2009.

[71] A. Burden, "How Should We Normalize Electromyograms Obtained from Healthy Participants? What We Have Learned from Over 25 Years of Research," Journal of Electromyography and Kinesiology, vol. 20(6), pp. 1023-1035, 2010.

[72] T. Moritani, F. D. Gaffney, T. Carmichael, and J. Hargis, Interrelationships Among Muscle Fibre Types, Electromyogram and Blood Pressure During Fatiguing Isometric Contraction. In Biomechanics, vol. IXA, International Series on Biomechanics, D. A. Winter, R. W. Norman, R. P. Wells, K. C. Hayes, and A. E. Patla, Eds. pp. 287-292. Champaign, Human Kinetics Publishers Inc., 1985.

[73] B. Gerdle, M.-L. Wretling, and K. Henriksson-Larsen, "Do the Fibre-Type Proportion and the Angular Velocity Influence the Mean Power Frequency of the Electromyogram?" Acta Physiol. Scand, vol. 134, pp. 341-346, 1988b.

[74] J. Elert, S. B. Rantapaa-Dahlqvist, K. Henriksson-Larsen, R. Lorentzon, and B. U. C. Gerdle, "Muscle Performance Electromyography and Fiber Type Composition in Fibromyalgia and Work-Related Myalgia," Scand. J. Rheumatol, vol. 21, pp. 29-34, 1992. 
[75] C. R. Ethier and C. A. Simmons, Introductory Biomechanics From Cells to Organisms. Cambridge University Press, 2007, pp. 332- 378.

[76] J. M. Wakeling and A. I. Rozitis, "Spectra properties of myoelectric signals from different motor uunit in the leg extensor muscles," The Journal of Experimental Biology, vol. 207, pp. 2519-2528, 2004. 